\title{
Optimal information loading into working memory in prefrontal cortex
}

\author{
Jake P. Stroud ${ }^{1}$, Kei Watanabe ${ }^{2}$, Takafumi Suzuki ${ }^{3}$, Mark G. Stokes ${ }^{4,5}$, Máté Lengyel ${ }^{1,6}$ \\ ${ }^{1}$ Computational and Biological Learning Lab, Department of Engineering, University of Cambridge, Cambridge, UK \\ ${ }^{2}$ Graduate School of Frontier Biosciences, Osaka University, Osaka, Japan \\ ${ }^{3}$ Center for Information and Neural Networks, National Institute of Communication and Information Technology, Osaka, Japan \\ ${ }^{4}$ Department of Experimental Psychology, University of Oxford, Oxford, UK \\ ${ }^{5}$ Oxford Centre for Human Brain Activity, Wellcome Centre for Integrative Neuroimaging, Department of Psychiatry, University of Oxford, \\ Oxford, UK \\ ${ }^{6}$ Center for Cognitive Computation, Department of Cognitive Science, Central European University, Budapest, Hungary \\ @ corresponding author: j.stroud@eng.cam.ac.uk
}

\begin{abstract}
Working memory involves the short-term maintenance of information and is critical in many tasks. The neural circuit dynamics underlying working memory remain poorly understood, with different aspects of prefrontal cortical (PFC) responses explained by different putative mechanisms. By mathematical analysis, numerical simulations, and using recordings from monkey PFC, we investigate a critical but hitherto ignored aspect of working memory dynamics: information loading. We find that, contrary to common assumptions, optimal information loading involves inputs that are largely orthogonal, rather than similar, to the persistent activities observed during memory maintenance. Using a novel, theoretically principled metric, we show that PFC exhibits the hallmarks of optimal information loading and we find that such dynamics emerge naturally as a dynamical strategy in task-optimized recurrent neural networks. Our theory unifies previous, seemingly conflicting theories of memory maintenance based on attractor or purely sequential dynamics, and reveals a normative principle underlying the widely observed phenomenon of dynamic coding in PFC.
\end{abstract}

Working memory involves the ability to temporarily hold information in mind, and it is essential to performing cognitively demanding tasks ${ }^{1,2}$. The neural mechanism of working memory maintenance is typically thought to rely on so-called attractor dynamics whereby synaptic interactions ('reverberations' ${ }^{3}$ ) between recurrently coupled neurons of a population maintain persistent patterns of activity even in the absence of external inputs ${ }^{4-6}$. Models based on attractor dynamics have successfully accounted for persistent neural activities exhibited by the prefrontal cortex (PFC) during working memory maintenance ${ }^{5-12}$. However, attractor networks appear poorly suited to also capture the widely observed rich transient dynamics exhibited by PFC (known as 'dynamic coding' 13-17) during a variety of working memory tasks ${ }^{18-24}$. We suggest that this is because work on attractor networks has focused on how such networks can maintain information, but not on how information should be loaded into these networks in the first place, for subsequent maintenance.

The widely established way to load information into attractor networks is to use inputs that are similar to the required persistent activity $6,7,10-12,25-28$. The ensuing dynamics then perform 'pattern completion' 27 : with the response of each neuron quickly reaching its steady-state and maintaining it throughout most of the delay period-unlike the diverse neural time courses seen in experiments that often exhibit long-lasting transients before settling to a steadystate ${ }^{13-16,24,29-32}$. Conversely, models that generate purely sequential activities without attractors can nat- urally capture these transients ${ }^{18,22,33,34}$ but fail to exhibit persistent activities. In order to account both for experimentally observed transient dynamics and persistent activities, a variety of additional purpose-built circuit mechanisms have been invoked for attractor networks ${ }^{11,12,20,35-38}$, suggesting that these dynamics may be epiphenomenal or serve altogether different roles than memory maintenance ${ }^{16,20,21,37,39-42}$. Here, we instead show that transient dynamics are a fundamental and functionally useful feature of information loading into attractor networks optimized for memory maintenance. Using a theoretically principled metric, we then demonstrate key signatures of optimal information loading in neural recordings from the lateral PFC (IPFC). Our results offer a novel, normative perspective on a core component of the operation of attractor networks-information loading-which has so far received little attention, and challenge long-held assumptions about pattern completion-like mechanisms in neural circuits.

\section{Results}

To understand the dynamics that underlie working memory maintenance, we considered the paradigmatic example of a memory-guided saccade task. In this task, subjects must maintain the location of 1 of 6 possible cues during a delay period after which they must respond with an eye movement to the correct location (Fig. 1a). In attractor network models of this task, the stimulus cue acts as a transient external input to a network of recurrently connected neu- 
a

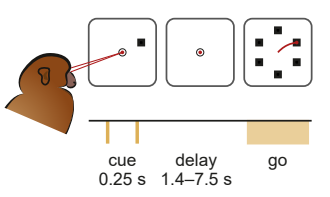

b

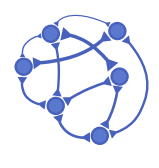

symmetric connections

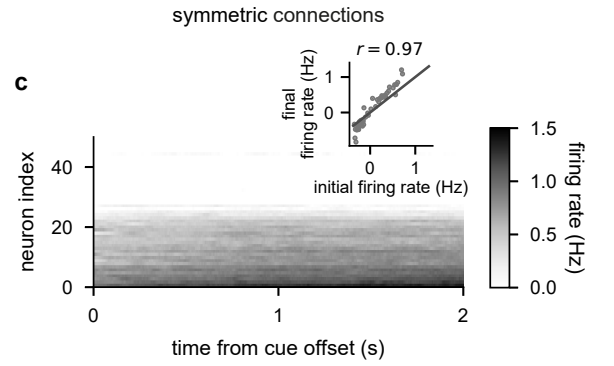

d

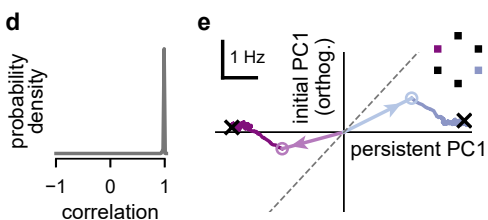

f

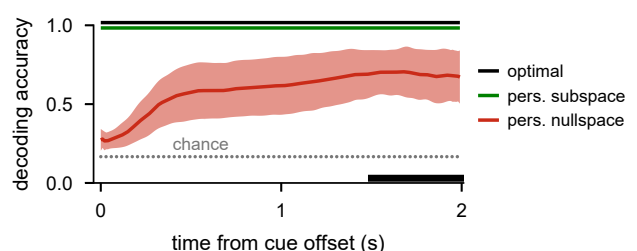

g unconstrained connections
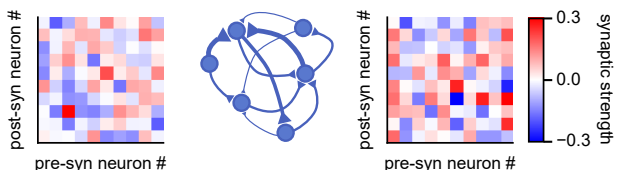

unconstrained connections

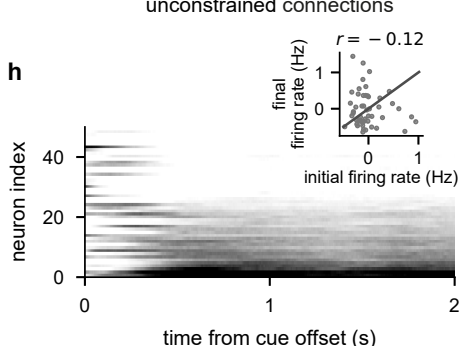

from cue offset (s)

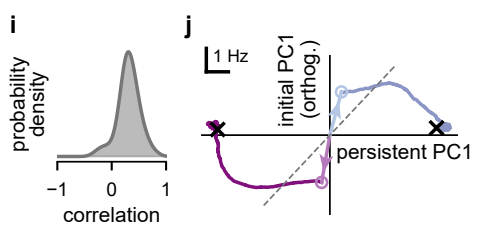

k

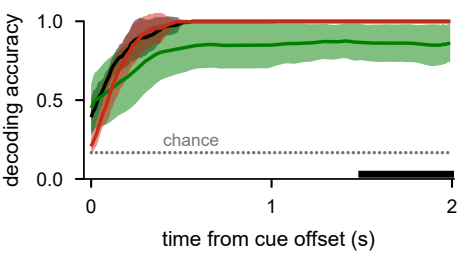

Fig. 1 | Pattern completion and optimal information loading in attractor networks. a, Illustration of the memory-guided saccade task. Time line of task events in a trial (bottom), with the corresponding displays (top). Top: black circle and squares show fixation ring, and the arrangement of visually cued saccade target locations, respectively (not to scale), red dots and line illustrate gaze positions during fixations and saccade, respectively. Bottom: yellow ticks show timing of stimulus cue onset and offset, yellow bar shows interval within which the go cue can occur. b, A network with symmetric connections. Left: network illustration. Right: the recurrent weight matrix for 10 of the 50 neurons. $\mathbf{c}-\mathbf{f}$, Analysis of neural responses in symmetric attractor networks (such as shown in b) with optimized initial conditions. c, Firing rate activity in a representative trial. Inset shows initial vs. final mean-centered firing rates across neurons (grey dots) in this trial and the Pearson correlation $(r ; p<0.001)$ between initial and final firing rates. Grey line is the identity line. $\mathbf{d}$, Distribution of Pearson correlations between initial and final mean-centered neural firing rates across all 6 cue conditions and 10 networks. e, Sub-threshold activity for 2 cue conditions in an example network (color trajectories). Open circles (with arrows pointing to them from the origin) show the optimized initial conditions, black crosses show stable fixed points, dashed grey line is the identity line. Horizontal axis (persistent PC1) shows network activity projected on to the 1st principal component (PC1) of network activities at the end of the delay period (across the 2 conditions shown), vertical axis (initial PC1 (orthogonalized)) shows projection to PC1 of initial network activities orthogonalized to persistent PC1. f, Performance of a delay-trained decoder (black bar indicates decoding training time period) on neural firing rate activity over time starting from optimized initial conditions with full optimization (black), or restricted to the 5-dimensional subspace spanning the 6 cue-specific attractors (persistent subspace, green), or the subspace orthogonal to that (persistent nullspace, red). Solid lines and shading indicate mean $\pm 1 \mathrm{~s} . d$. across all 6 cue conditions and 10 networks. Grey dotted line shows chance level decoding. Green and black lines are slightly offset vertically to aid visualization. $\mathbf{g}$, Same as b but for an attractor network with unconstrained connections. $\mathbf{h}-\mathbf{k}$, Same as $\mathbf{c}-\mathbf{f}$, for attractor networks with unconstrained connections. The Pearson correlation in $\mathbf{h}$ (inset) is not significant $(p>0.4)$.

rons. This external input drives the network into a suitable state from which its intrinsic dynamics, in the absence of the cue, are 'attracted' into a distinct cuespecific steady state, as neurons continue stimulating one-another through their strong recurrent connections. This mechanism allows the network to stably maintain the memory of the cue in a persistent pattern of neural activities.

Standard approaches to studying attractor networks used models in which the connectivity between neurons was constrained to be symmetric ${ }^{7,10,25-27,43-46}$, making the analysis of their dynamics mathematically more convenient ${ }^{26,27,45,47,48}$. Thus, we first replicated results with such symmetric networks that were optimized to perform the working memory task shown in Fig. 1a. For simplicity, we only modelled the intrinsic dynamics of the network during the delay period and the effect of the cue was captured by cue-specific initial neural activities (i.e. neural activities at the beginning of the delay period ${ }^{27,43,44}$; Fig. 1b). To study optimal information loading, we optimized these initial activities (with a constraint on their magnitude; Methods) in order to maximize the performance of the network, as determined by how well the cue could be decoded from neural activities at the end of the delay period. In other words, we asked where (in neural state space) the dynamics of the network need to start from so as to consequently generate a robustly identifiable, cuespecific pattern of persistent activity. 
bioRxiv preprint doi: https://doi.org/10.1101/2021.11.16.468360; this version posted December 17, 2021. The copyright holder for this preprint (which was not certified by peer review) is the author/funder, who has granted bioRxiv a license to display the preprint in perpetuity. It is made available under aCC-BY 4.0 International license.

\section{Pattern completion and optimal information load- ing in attractor networks}

We found that optimal initial activities gave rise to classical pattern completion dynamics in symmetric networks. First, initial activities were noisy versions of (and in fact highly similar to) the desired persistent patterns (Fig. 1c inset, and Fig. 1d). Second, the ensuing dynamics were driven directly into the corresponding steady state resulting in only small and gradual changes in activities over the delay period (Fig. 1c). Further analysis of these dynamics showed that the optimal initial activities aligned well with directions in neural state space that best distinguished between the desired persistent activities (Fig. 1e, 'persistent PC1' component of pale arrows and circles; Extended Data Fig. 1b), with only a comparably small component in orthogonal directions specific to these initial activities (Fig. 1e, 'initial PC1 (orthogonalized)') which subsequently changed little over time (Fig. 1e, dark trajectories). As a result, a decoder based on templates of neural activity during the late delay period (i.e. during the steady state of the network; Fig. 1f, black bar on time axis), generalized well to all times and was able to decode the cue identity from neural activities with high accuracy throughout the delay period (Fig. 1f, black line). We found that the similarity between initial and persistent activities was critical for these networks. When constrained to use initial activities that were orthogonal in neural state space to persistent activities (i.e. lying in the 'persistent nullspace'), these networks performed substantially more poorly at all times (Fig. 1f, red line) and activity often did not settle into the correct attractor state (Extended Data Fig. 1d). In contrast, explicitly enforcing these networks to use initial activities that were similar to persistent activities (i.e. lying in the 'persistent subspace') did not compromise their performance (Fig. 1f, green line; Extended Data Fig. 1c).

In contrast to networks artificially constrained to have symmetric connections between neurons, we found that attractor networks optimized to perform the task shown in Fig. 1a without such a constraint exhibited dynamics distinctly unlike simple pattern completion (Fig. 1g-k). First, initial activities resembled persistent activity much less than in symmetric networks (Fig. 1i), such that their correlation could even be negative (Fig. $1 \mathrm{~h}$ inset). Second, neural activities often underwent substantial and non-monotonic changes before ultimately settling into an attractor state (Fig. 1h). This was also reflected in optimal initial activities being strongly orthogonal to persistent activities (Fig. 1j, pale arrows; Extended Data Fig. 1f), with this orthogonality decaying over the delay period (Fig. 1j, dark trajectories). Third, a decoder trained on neural activity from the late delay period generalized poorly to early times (Fig. 1k, black line), thus exhibiting a fundamental signature of 'dynamic coding' ${ }^{13-16}$. We found that the orthogonality of initial conditions in these networks was instrumental for high performance: in a double dissociation from symmetrically constrained networks, restricting initial conditions to be in the persistent sub- space (Fig. 1k, green line; Extended Data Fig. 1g), but not in the persistent nullspace (Fig. 1k, red line; Extended Data Fig. 1h), diminished decodability at the end of the delay period (cf. Fig. 1f).

\section{Dynamical analysis of optimal information loading}

To understand why optimal information loading in symmetric versus unconstrained attractor networks is so different, and in particular why inputs orthogonal to attractor states are optimal for unconstrained networks, we reduced these networks to a canonical minimal model class consisting of only two neurons ${ }^{44,50,51}$. While attractor network dynamics in general rely on the activation functions ( $\mathrm{f}-\mathrm{I}$ curves) of neurons being nonlinear $8,25,27,45$, for analytical tractability, we considered networks with linear dynamics (i.e. in which neurons had linear activation functions). Critically, with the appropriate set of synaptic connections, even linear networks can exhibit persistent activity $11,12,26,44,48,52$ the key feature of working memory maintenance in attractor networks. In addition, we were able to show that the insights gained from the analysis of such linear models also apply to the behavior of nonlinear systems (Methods, Extended Data Figs. 2 and 3, see also Supplementary Math Note).

For our analyses, we again distinguished between models with symmetric connectivity between neurons (Fig. 2a; top) ${ }^{26,44,51}$, and models without this constraint (Fig. 2a; bottom) $)^{11,12}$. The dynamics of these networks are fully described in a two-dimensional state space spanned by the activities of the two neurons (Fig. 2b) and define a flow-field in this space determining how changes in neural activities depend on the network state (Fig. 2b; blue arrows). While the persistent subspace of nonlinear networks can consist of a number of discrete attractor states corresponding to distinct patterns of persistent activity (Fig. 1 and Extended Data Figs. 1 and 2, Methods), linear attractor networks express a continuum of persistent activity patterns ${ }^{11,12,26,44}$. In either case, attractor networks encode stimulus information in the location of the state of the network within the persistent subspace. In our two-neuron linear network, the persistent subspace simply corresponds to a line onto which the flow field converges (Fig. 2b; green lines). Therefore, the persistent mode of our network is its 'coding direction' 25 , which allows it to distinguish between two stimuli depending on which side of the origin the state of the network is. The larger the magnitude of its activity along this mode at the end of the delay period, the more robustly the identity of the stimulus can be decoded (e.g. in the presence of noise). This is analogous to noisy nonlinear attractor networks, in which the further 'out' the network is along the coding direction between two attractors ${ }^{25}$, the more likely the activity is to eventually fall into the correct attractor. Consistent with this, we found a close correspondence between decoding accuracy in such noisy nonlinear attractor networks, and this measure of alignment with the most persistent mode (Extended Data Fig. 3a-c, see also Supplementary Math Note). 

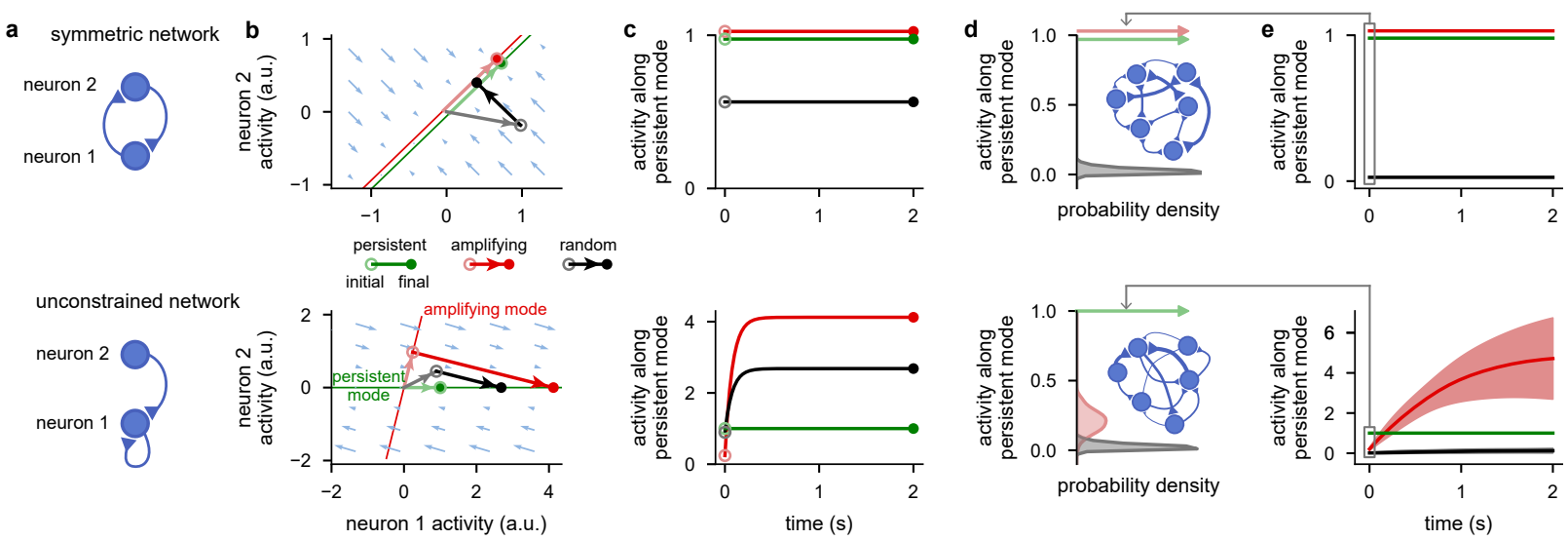

Fig. 2 | Dynamical analysis of optimal information loading. a, Architecture of a symmetric (top) and an unconstrained network (bottom; Methods). (Note that any two-neuron network with unconstrained connectivity can be equivalently expressed as a network with a single effective feedforward connection and recurrent self loops, as shown here ${ }^{18,49}$.) b. Neural state space of the symmetric (top) and unconstrained network (bottom). Pale blue arrows show flow field dynamics (direction and magnitude of movement in the state space as a function of the momentary state). Thin green and red lines indicate the persistent and most amplifying modes (Methods), respectively (lines are offset slightly in the top panel to aid visualisation). Pale green, red, and grey arrows with open circles at the end indicate persistent, most amplifying, and random initial conditions, respectively. Dark green, red, and black arrows show neural dynamics starting from the corresponding initial condition. (Green arrows, and the red arrow in the top panel cannot be seen, as no movement in state space happens from those initial conditions.) Filled colored circles indicate final (persistent) neural activity. c, Time course of network activity along the persistent mode (i.e. projection onto the green line in b) when started from the persistent (green), most amplifying (red), or random initial conditions (black) for the symmetric (top) and the unconstrained model (bottom). $\mathbf{d}$, Distributions of absolute overlap with the persistent mode for persistent (pale green), most amplifying (pale red), or random initial conditions (grey) across 100 randomly sampled 1000-neuron symmetric (top) or unconstrained networks (bottom; Methods). For the symmetric models, the persistent and most amplifying initial conditions produce delta functions at 1 (arrows). Insets show illustration of large networks of neurons with either symmetric (top) or unconstrained (bottom) connections. e, Time course of absolute overlap with the persistent mode when starting network dynamics from persistent (green), most amplifying (red), or random initial conditions (black) for the symmetric (top) and the unconstrained network (bottom). Lines and shaded areas show mean \pm 1 s.d. over the 100 randomly sampled 1000-neuron networks from $\mathbf{d}$.

To understand the mechanisms of information loading, we considered three distinct stimulus directions in which inputs can offset the state of the network from the origin (i.e. the background state of the network before stimulus onset). We then analysed the time course of the network's activity along the persistent mode ${ }^{11,12,25}$ after being initialised in each of these directions. First, we considered inputs aligned with the persistent mode, the input direction studied in classical attractor networks ${ }^{11,12,26,44,51}$ (Fig. 2b; pale green arrows and open circles). Second, we considered the "most amplifying mode", which is defined as the stimulus direction that generates the most divergent and thus best discriminable activity over time ${ }^{53-56}$ (Methods; Fig. 2b, red lines, and pale red arrows and open circles). Third, we considered a random input direction (Fig. 2b; grey lines/circles).

We were able to show mathematically that optimal information loading, in the sense of maximising overlap with the persistent mode at long delays, is always achieved with inputs aligned with the most amplifying mode (regardless of whether the connectivity is symmetric or not; Methods and Supplementary Math Note). In symmetric networks, the most amplifying mode is aligned with the most persistent mode (Fig. 2b; top) ${ }^{28,57}$, and thus does not generate activity transients (Fig. 2c; top)-accounting for the simple pattern completion dynamics seen in classical attractor networks with symmetric connectivity 7,10,25-27,43-45 (Fig. 1b-f). However, in unconstrained networks, the most amplifying mode is typically different from the most persistent mode (Fig. 2b; bottom). Intuitively, this is because feeding neuron 1 (the persistent mode) indirectly through the feed-forward connection from neuron 2 can increase its activity more than just feeding it directly ${ }^{18,28,33,49,50}$ (Fig. 2a,b; bottom). This means that activity evolving from the most amplifying mode exhibits a distinct transient behaviour: its overlap with the most persistent mode is initially low and then increases over time (Fig. 2c; bottom, red line), accounting for the richer transients seen in unconstrained attractor networks (Fig. 1g-k). Although our mathematical analysis only applied to linear dynamics, we found that the same principles applied to the dynamics of a canonical nonlinear attractor system, when the persistent and most amplifying directions were defined locally around its ground state (Methods; Extended Data Fig. 2, see also Supplementary Math Note).

These insights also generalised to large networks, with more than two neurons (Fig. 2d,e and Extended Data Fig. 4). This is because in large unconstrained networks, there are many effectively feed-forward motifs embedded in the full recurrent connectivity of the circuit which can all contribute to transient amplification ${ }^{18}$. Moreover, in these networks, the most amplifying direction becomes increasingly orthogonal to the most persistent mode ${ }^{56}$ (Extended Data Fig. 4a, bottom). Thus, the effects seen in 2-neuron networks are further accentuated in large unconstrained networks ${ }^{56}$ (Fig. 2d,e; bottom, red vs. green), but not in symmetric networks (Fig. 2d,e; top), while random initial conditions become fully orthogonal in both networks and result in poor overlap with the persistent mode (Fig. 2d,e; black). Numerical simulations of large, ran- 
domly connected, noisy networks corroborated the results of our mathematical analysis: explicitly optimizing a network's initial condition so as to generate persistent activity made it overlap strongly with the most amplifying mode (Extended Data Fig. 4c). Moreover, large nonlinear neural networks also showed a similar pattern of results (Extended Data Fig. 3a-c, see also Supplementary Math Note).

\section{Neural signatures of optimal information loading}

Our dynamical analysis suggested that there should be clearly identifiable neural signatures of a network performing optimal information loading. To demonstrate this, and to allow a more direct comparison with data, we used the same large, randomly connected, noisy linear networks that we analysed earlier (Extended Data Fig. 4c-d) which could be either symmetric or unconstrained, with the cue period (Fig. 3, yellow ticks and lines) modelled using temporally extended constant inputs, mimicking typical experiments $s^{7,16,29,58}$. We studied the three different information loading strategies that we identified earlier: inputs aligned with either the persistent mode, the most amplifying mode, or a cue-specific random direction (Fig. 3). We began by conducting a decoding analysis using templates of late delay activity, as is often done for prefrontal cortical recordings $11,13,14,16,32,59$ (and also in Fig. 1f,k). We first verified that for a fixed level of neuronal noise, the most amplifying inputs were indeed optimal for achieving high decodability at the end of the delay period (Fig. 3a, red lines). In contrast, random inputs in both types of networks performed considerably more poorly (Fig. 3a, grey lines). Remarkably, persistent inputs achieved a similarly low level of decodability at late delay times in unconstrained networks (Fig. 3a, bottom; compare pale green and grey lines) - but not in symmetric networks in which they were identical to the most amplifying input (Fig. 3a, top; overlapping green and red lines). In contrast, as expected, decodability early in the trial was best with persistent inputs (Fig. 3a, bottom; compare red and pale green lines). Thus, as shown by our original analysis of nonlinear attractor networks (Fig. 1k), optimal information loading actually traded off high decoding performance at later times for poorer performance at the beginning of the trial.

The level of noise in the networks we have studied so far was not constrained by data, which typically shows high decodability ${ }^{11,13,14,16,32,59}$. This is important because the sub-optimal input conditions could achieve high decoding performance by appropriately reducing the noise level in our simulations (Fig. 3a, asymptotic values of dark green and black lines). Thus, asymptotic decoding performance alone cannot be used to identify the information loading strategy employed by a network. To address this, in subsequent analyses, we used networks in which the level of late-delay performance was matched between the three information loading strategies by appropriately titrating the level of noise. Nevertheless, a critical difference emerged between the different information loading strategies even in these 'performance-matched' networks -at least in those with realistic, unconstrained connectivity. The delay-trained decoder only performed well when tested late in the delay period for both random and most amplifying input directions (Fig. 3a, bottom; black and red lines), whereas for inputs aligned with the persistent direction this decoder performed near ceiling at all times after cue onset (Fig. 3a, bottom; dark green line). These results indicate that the level and dynamics of decoding performance achieved by different inputs in non-linear attractor networks (Fig. 1f,k) were explained by their overlap with the most amplifying modes of those networks (Extended Data Fig. 3a-c).

Next, in order to more fully characterise the differences between persistent versus random or most amplifying inputs, and for a comprehensive comparison with experimental data ${ }^{13,14,16,32,59}$, we employed full crosstemporal decoding, which uses all combinations of times during the trial when the decoder is trained and tested (Fig. 3b). This analysis showed that all information loading strategies led to dynamics in which stimulus information was present at all times after cue onset (Fig. 3b, diagonals are all black). Moreover, in symmetric networks, and for the persistent mode inputs in the unconstrained network, stimulus information was maintained using a 'stable code'13,15,16,21 (Fig. 3b, top, and bottom left, all off-diagonals are black). Earlier attractor network models of working memory ${ }^{7,10-12,26}$ also showed such stable coding (Extended Data Fig. 5 and Extended Data Fig. 10d,e). In contrast, in the unconstrained network, random and most amplifying mode inputs led to poor decodability between early and late time points after cue onset (Fig. 3b, bottom; center and right, off-diagonals indicated by pink rectangles are white/grey), suggesting sequential activities during the early-to-late delay transition ${ }^{18,33,34}$, and giving rise to the phenomenon of 'dynamic coding' 13-16,21,32. These activities were then stabilised during the late delay period as the network dynamics converged to a persistent pattern of activity (Fig. 3b, bottom; center and right, off-diagonals inside cyan squares are black). As a comparison, earlier network models of working memory based on purely sequential activities ${ }^{18,33}$ also predicted low cross-temporal generalization at early times. However, the dynamics of such models never settle into a persistent pattern of activity and thus there is never any cross-temporal generalization during the delay period (Extended Data Fig. $5 d, h, i, j)$. In sum, these decoding analyses were able to clearly distinguish between persistent mode and other types of inputs, but not between random and amplifying inputs.

To construct a targeted measure for identifying networks using most amplifying inputs, we exploited the fact that in large networks, random inputs are almost certainly guaranteed to have negligible overlap with any other direction in neural state space, including the most amplifying mode. Thus, we directly measured the time courses of the overlap of neural activities with the top $25 \%$ most amplifying modes. We quantified this overlap as the fraction of variance of neural activ- 

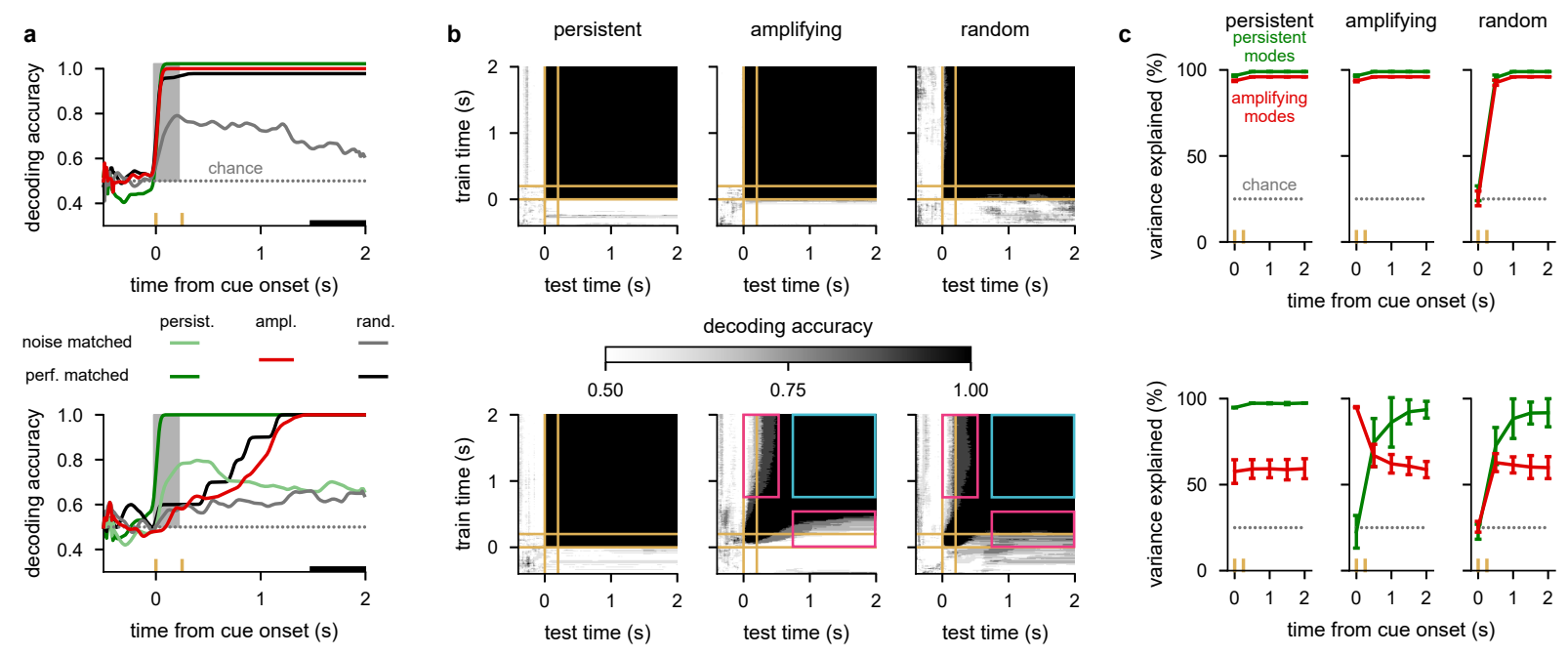

Fig. 3 | Neural signatures of optimal information loading. a, Performance of a delay-trained decoder (black bar indicates decoder training time period; Methods) on neural activity over time. Two cue conditions were used with inputs that were identical but had opposite signs. Lines show mean across 10 random 100-neuron linear symmetric networks (top) and unconstrained networks (bottom). Yellow ticks on horizontal axis indicate cue onset and offset times and the vertical grey bar indicates the cue epoch. We show results for inputs aligned with the persistent mode (dark and pale green), the most amplifying mode (red), or a random direction (black and grey). Light colors (pale green and grey, 'noise-matched') correspond to networks with the same level of noise as in the reference network (red), while dark colors (dark green and black, 'performance-matched') correspond to networks with the same level of asymptotic decoding performance as that in the reference network. Grey dotted line shows chance level decoding. Green, red, and black lines are slightly offset vertically in the top panel to aid visualization. b, Cross-temporal decoding of neural activity for the 3 different information loading strategies (persistent, most amplifying, and random respectively in left, center, and right panels) for a representative symmetric network (top) and unconstrained network (bottom) from those shown in a. Yellow lines indicate cue onset and offset times. Pink rectangles indicate poor generalization between time points (i.e. dynamic coding) and cyan rectangles indicate examples of good generalization between time points (i.e. stable coding). c, Percent variance explained by the subspace spanned by either the $25 \%$ most persistent (green) or $25 \%$ most amplifying (red) modes as a function of time in the same symmetric (top) and unconstrained networks (bottom) analyzed in a. Lines and error bars show mean \pm 1 s.d. across networks. We show results for inputs aligned with the persistent mode (left), most amplifying mode (center), or a random direction (right). Grey dotted line shows chance level overlap with a randomly chosen subspace occupying $25 \%$ of the full space.

ities that these modes collectively explained (Fig. 3c, red lines; Methods). For a comparison, we also measured these time courses for the overlap with the top $25 \%$ most persistent modes (Fig. 3c, green lines). (We used the top set of amplifying and persistent directions, rather than just a single direction in each case, because large networks can harbor multiple directions that represent similarly persistent activity patterns and similarly efficient amplifying modes, respectively ${ }^{53,54}$.)

As expected, for symmetric networks, persistent and equivalent amplifying mode inputs resulted in both overlaps being high immediately from stimulus onset (Fig. 3c, top; left and center). Random inputs started with chance overlap which increased over time to ceiling as the network settled into its persistent (or, equivalently, most amplifying) mode (Fig. 3c, top right). In unconstrained networks, persistent inputs led to constant high and moderate overlaps with the persistent and most amplifying modes, respectively (Fig. 3c, bottom left). Random inputs again started with chance overlap for both modes, which then increased to the same levels that are characteristic of the persistent mode (Fig. 3c, bottom right). In contrast, most amplifying inputs were uniquely characterised by a crossover between the time courses of the two overlap measures. Initially, neural activities overlapped strongly with the most amplifying mode (by construction), but showed only chance overlap with the persistent mode (Fig. 3c, bottom middle). Over time, these overlap measures changed in opposite directions, such that by the end of the delay period overlap was high with the persistent mode and lower with the most amplifying mode (Fig. 3c, bottom middle). Therefore, the cross-over of these overlap measures can be used as a signature of optimal information loading utilising inputs aligned with the most amplifying mode. Indeed, while cross-temporal decoding in an earlier network model of working memory ${ }^{11}$ with inputs aligned with persistent and random directions could in principle show similar patterns to that achieved by the most amplifying mode (Extended Data Fig. 5d-e), the overlap measures that we developed here clearly revealed the lack of optimal information loading in that model (Extended Data Fig. 5f). In addition, we confirmed in numerical simulations that the same signature of optimal information loading remains detectable even under the practical constraints of experimental data analysis: when the underlying network dynamics is nonlinear, and only accessible indirectly by fitting linear dynamical models to the neural responses they generate (Extended Data Fig. 3d, Methods and Supplementary Math Note).

\section{Signatures of optimal information loading in mon- key IPFC}

To study whether the PFC shows the dynamical signatures of optimal information loading that our theoretical analyses identified, we performed multi-channel recordings of the lateral prefrontal cortex (IPFC) in two monkeys during a variable-delay memory-guided sac- 

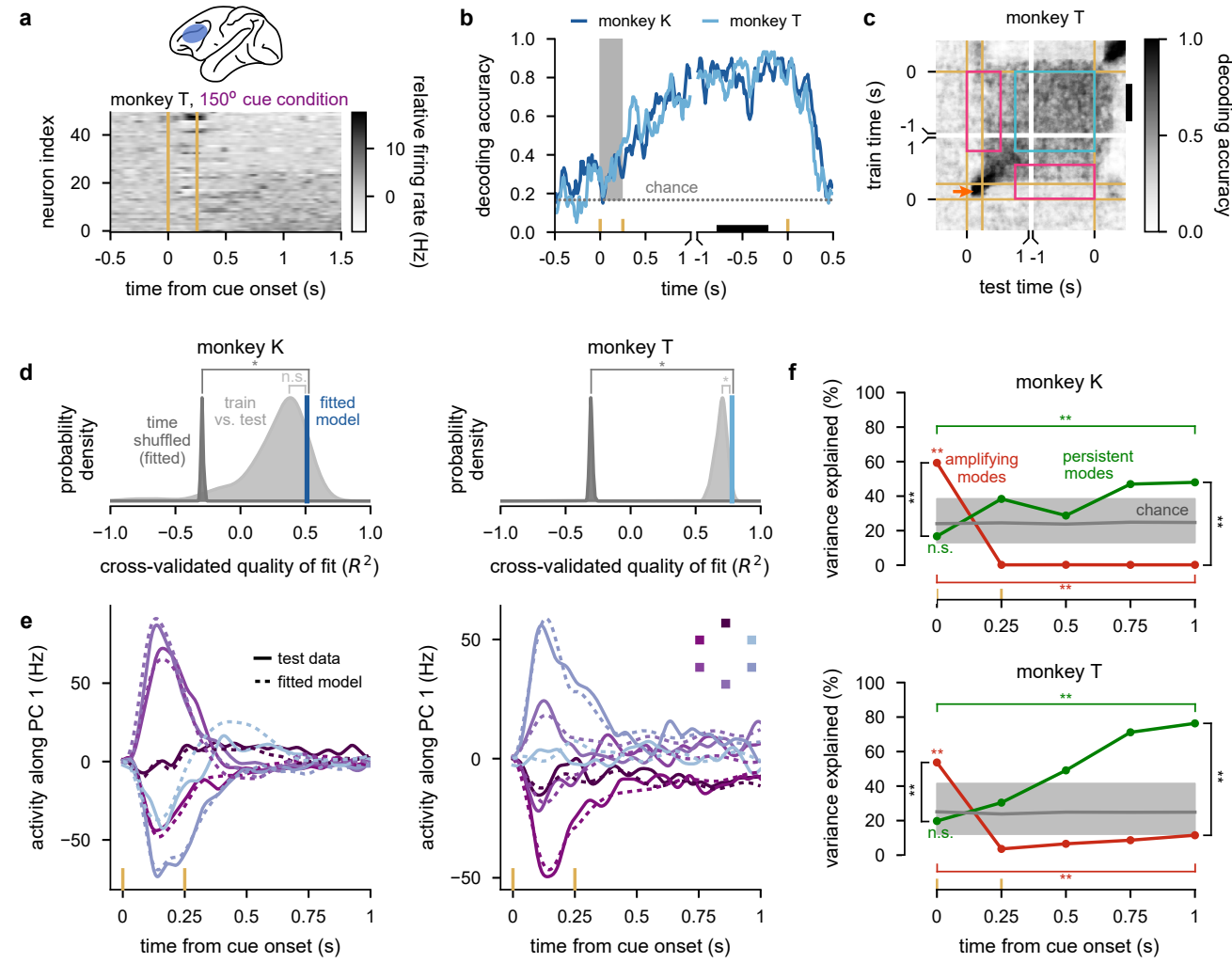

Fig. 4 | Signatures of optimal information loading in monkey IPFC. a, Top: IPFC recording location. Bottom: neural firing rates (relative to the time-dependent but condition-independent mean) for one stimulus cue condition for 50 example neurons. See Fig. 1a (and Methods) for experimental paradigm. Neurons are ordered according to their firing rate at the end of the period shown. Vertical yellow lines indicate stimulus cue onset and offset. b, Performance of a delay-trained decoder (black bar indicates decoder training time period; Methods) on neural activity over time. Yellow ticks on horizontal axis indicate stimulus cue onset, offset, and go cue times, and the vertical grey bar indicates the stimulus cue epoch. Data is aligned to either stimulus cue onset (first $1.5 \mathrm{~s}$ ) or to the go cue (final $1.5 \mathrm{~s}$ ). Grey dotted lines show chance level decoding. c, Cross-temporal decoding of neural activity for monkey T (see Extended Data Fig. 10a for Monkey K). Yellow lines indicate stimulus cue onset, offset, and go cue times. Pink rectangles indicate poor generalization between time points (i.e. dynamic coding) and the cyan rectangle indicates examples of good generalization between time points (i.e. stable coding). The orange arrow indicates good same-time decoding during the cue epoch. The black vertical bar on the right indicates the delay-trained decoder training time period from b. d, Cross-validated quality of fits on 10 different held-out data splits when fitting 20-dimensional linear dynamical systems to neural activity (blue) and time shuffled controls (dark grey) (Methods). We also show quality of fits of the data against itself ('test vs. train'; light grey). e, Neural activity for each of the 6 cue conditions projected onto the top PC (solid lines) for monkey K (top) and monkey T (bottom). Solid lines show held-out test data from 1 split, dashed lines show predictions of fitted model dynamics. The inset for monkey $T$ shows which color corresponds to each cue condition. f, Percent variance explained by the subspace spanned by either the $25 \%$ most persistent (green) or $25 \%$ most amplifying (red) modes as a function of time for the 20-dimensional linear dynamical systems fitted to data from monkey $\mathrm{K}$ (top) and monkey $\mathrm{T}$ (bottom). Grey lines show chance level overlap defined as the expected overlap with a randomly chosen subspace occupying $25 \%$ of the full space (median and 95\% C.I. across 200 random subspaces). Comparisons shown in $\mathbf{d}$ and $\mathbf{f}$ use two-sided permutation tests ${ }^{*}$, $p<0.05 ;{ }^{* *}, p<0.01 ;$ n.s., not significant; see Methods)

cade task (Fig. 1a). Our recordings yielded 438 and 625 neurons (for monkeys $\mathrm{K}$ and $\mathrm{T}$, respectively; Extended Data Fig. 9, Methods). We analysed the population dynamics of all recorded neurons in each monkey and applied the same metrics to this dataset that we applied to our models. Population dynamics appeared to show rich transient dynamics during the cue and early delay period, followed by relatively stable dynamics during the late delay period (Fig. 4a). This was reminiscent of the dynamics we found in unconstrained attractor networks following optimal information loading (Fig. 1h).

To further quantify this behaviour, we conducted decoding analyses. First, in line with previous studies $^{13,14,16}$, we found that a delay-trained decoder did not generalise to times outside of the delay period and in particular showed near-chance performance during the cue period (Fig. 4b). This was distinct from the pattern completion dynamics seen in classical attractor network models of working memory (Fig. 1f,k green; Fig. 3a green; Extended Data Fig. 5e), but similar to that expected from random or optimal inputs in unconstrained networks (Fig. 1k black and red; Fig. 3a bottom, black and red; Extended Data Fig. 5e, orange dotted).

Full cross-temporal decoding reinforced these results: decoders trained during the delay epoch did not generalise to the cue or go epochs and vice versa (Fig. 4c and Extended Data Fig. 10a, pink rectangles). Thus, the neural activity exhibits dynamic coding ${ }^{13,14}$ rather than the stable coding characteristic of simple pattern completion (Fig. 3b top, and bottom left; Extended Data Fig. 5a-b; and Extended Data Fig. 5d, left). Importantly, same-time decoding performance was close to 1 throughout the cue and delay epochs (Fig. 4c and Extended Data Fig. 10a, orange arrow). This confirmed that the poor cross-temporal generalisation between early and late periods of a trial was 
not because the cue information had not yet reached PFC, or was maintained by activity-silent mechanisms as previously suggested $21,36,60$. At the same time, also in line with previous studies ${ }^{13-16,32}$, we found relatively stable coding during the late delay period (Fig. 4c and Extended Data Fig. 10a, cyan square). This ruled out purely sequential activity-based dynamics $^{18,33,61-63}$ (Extended Data Fig. 5c).

Quantifying the relative alignment of the subspaces occupied by neural dynamics across time ${ }^{11,64}$ using PCA confirmed the orthogonality of neural activities between different task epochs (Extended Data Fig. 10b-c). Further analyses showed that this orthogonality was not simply due to distinct sub-populations of neurons being active in different task epochs, but was instead largely due to changes in population-wide activities patterns $^{16,39}$ (Extended Data Fig. 10d-e).

These results, in line with previous findings ${ }^{14-16,32}$, clearly indicated that activities during the cue period were largely orthogonal from those during the delay period. However, these analyses alone were unable to distinguish between two fundamentally different information loading strategies PFC could employ: random input directions, or optimal information loading using specifically amplifying directions. Thus, in order to clearly identify the information loading strategy underlying the combination of dynamic and stable coding that we found, we applied our overlap measure (Fig. 3c) to these PFC recordings. For this, we first fitted a 20-dimensional linear dynamical system model to the cue and early delay epochs of our recordings (0-1 s after cue onset, Methods). We confirmed that linear dynamics provided a reasonably accurate cross-validated fit to the data compared to a time shuffled control (which destroyed the lawful dynamics of the data; Fig. 4d, dark grey, see also Methods), and model-free train vs. test performance (which indicated that cross-validated errors were mostly due to sampling noise differences between the train and test data; Fig. 4d, light grey) and recapitulated the most important aspects of the trial-average dynamics in each condition (Fig. 4e). We then performed the same overlap analysis on the fitted linear dynamics of the data that we used on our simulated networks with linear dynamics (Fig. 3c; Methods). As expected from our decoding analyses (Fig. 4b,c), the overlap of neural activities with the most persistent modes was at chance initially and gradually increased (Fig. 4f, green and Extended Data Fig. 10h). Critically however, the overlap of neural activities with the most amplifying modes was high initially and decreased with time (Fig. 4f, red and Extended Data Fig. 10h). We also noted that the overlap with the most amplifying directions became significantly lower than chance over time. This suggests that PFC circuits may be more mathematically 'non-normal' (i.e. include stronger feedforward loops ${ }^{18,33}$, or excitatory-inhibitory interactions ${ }^{53}$ ) than the networks with randomly chosen weights that we used in Fig. $3^{53,56}$. As a control, repeating the same analyses on time-shuffled data, or on data taken from the late delay period (when the network should already be near an attractor state) did not result in the same cross-over pattern. In particular, there was a high initial overlap with the most persistent modes and a chance (or below chance) initial overlap with the most amplifying modes (Extended Data Fig. 10f-h).

Therefore, these analyses provide strong experimental evidence that PFC circuit dynamics utilize optimal information loading with inputs aligning with the most amplifying modes (compare to Fig. 3c; bottom middle and Extended Data Fig. 10h, third vs. fourth row) rather than simply using random input directions (compare to Fig. 3c; bottom right and Extended Data Fig. 10h, first vs. fourth row).

\section{Information loading in task-optimized non-linear networks}

Our definition of optimal information loading relied on full access to the algebraic form of the dynamics of a network, something that animals will not have when performing a working memory task. Thus, an important question is whether optimizing simple taskrelevant cost functions ${ }^{30,65,66}$, that do not explicitly encourage optimal information loading, can be sufficient for the underlying circuits to adopt an optimal information loading strategy. For this, we trained (nonlinear) recurrent neural networks (without connectivity constraints Fig. 5a; Methods) on the same memoryguided saccade task as that which our animals performed (Fig. 1a). Specifically, during the cue epoch, these networks received temporally extended input from one of six input channels on any given trial depending on the cue condition (Fig. 5a, left). Similarly, network activity was decoded into one of six possible behavioural responses via six readout channels (Fig. 5a, right). All recurrent weights in the network, as well as weights associated with the input and readout channels, were optimized.

We compared three ways in which this task could be operationalized in the cost function that these networks needed to optimize. Networks were required to stably maintain cue information either immediately after cue onset, just in time before the go cue appeared, or only at response time (Fig. 5b). Specifically, the first and last one of these three cost functions were analogous to previous proposals: models performing evidence accumulation of noisy stimuli typically represent the accumulated stimulus evidence in a stable format as soon as all stimulus information has been received $^{30,67}$, while other models attempt to encode the motor response and thus only require a stable encoding of cue identity at response time $22,65,66$. In comparison, our novel just-in-time cost was motivated by our finding that optimal information loading trades off higher performance later in the trial for lower performance early on in unconstrained networks (Fig. 1k and Fig. 3a, bottom).

Training networks for all three cost functions was successful, and resulted in high performance by a latedelay decoder already by the end of the cue epoch for the cue-delay cost (Fig. 5c and Extended Data Fig. 6a, 
a

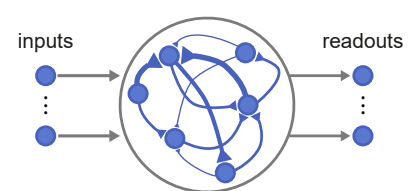

c

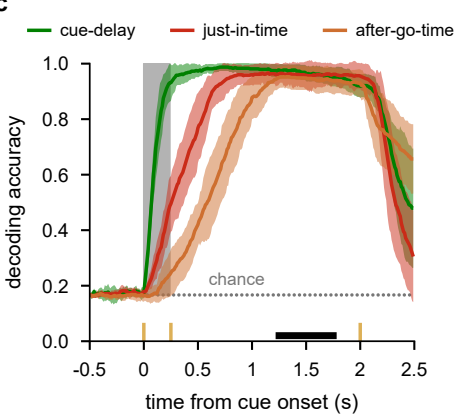

b
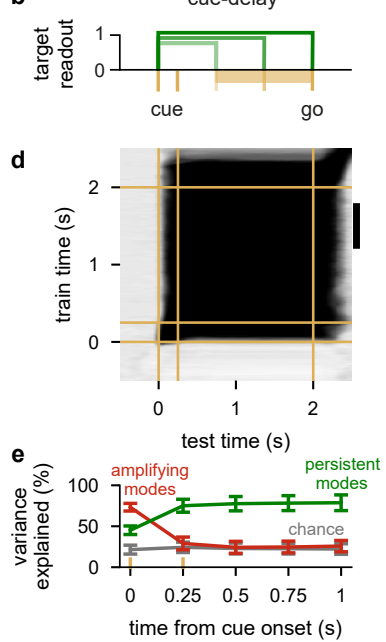

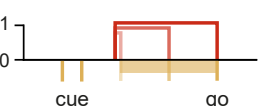

go
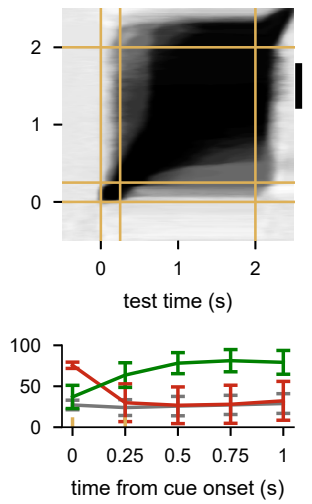

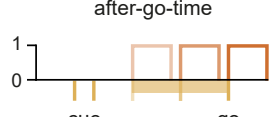

go

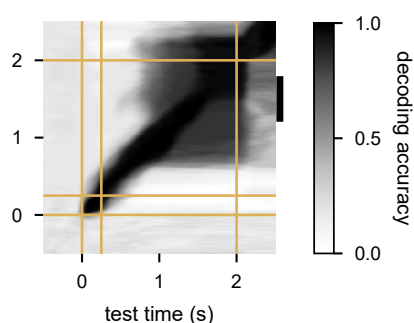

test time $(\mathrm{s})$

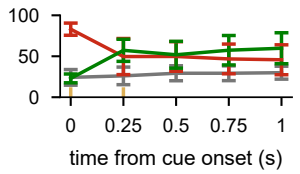

Fig. 5 | Information loading in task-optimized networks. a, Illustration of a recurrent neural network model with unconstrained connectivity (middle). During the cue epoch, networks received input from one of six input channels on any given trial depending on the cue condition (left). Network activity was decoded into one of six possible behavioural responses via six readout channels (right). All recurrent weights in the network (50 neurons), as well as weights associated with the input and readout channels, were optimized (Methods). b, Illustration of cost functions used for training. Yellow ticks indicate cue onset and offset times, yellow bars indicate range of go times in the variable delay task. Boxcars show intervals over which stable decoding performance was required in three example trials with different delays for each of the cost functions considered (Methods): cue-delay (left), just-in-time (center), or after-go-time (right). c, Performance of a delay-trained decoder (black bar indicates decoder training time period; Methods) on model neural activity over time in trials with a $1.75 \mathrm{~s}$ delay. Yellow ticks show stimulus cue onset, offset, and go times, and the vertical grey bar indicates the cue epoch. Neural activities were generated by networks optimized for the cue-delay (green), just-in-time (red), or after-go-time (orange) costs. Solid lines and shading indicate mean \pm 1 s.d. across 10 networks. Grey dotted line shows chance level decoding. d, Cross-temporal decoding of model neural activity for cue-delay (left), just-in-time (center), and after-go-time (right) trained models. Yellow lines indicate stimulus cue onset, offset, and go times. The black vertical bar on the right indicates the delay-trained decoder training time period from c. e, Percent variance explained by the subspace spanned by either the $25 \%$ most persistent (green) or $25 \%$ most amplifying (red) modes as a function of time for 20-dimensional linear dynamical systems fitted to the model neural activities of networks optimized for the cue-delay (left), just-in-time (center), or after-go-time cost (right). Grey lines show chance level overlap defined as the expected overlap with a randomly chosen subspace occupying $25 \%$ of the full space. Lines and error bars show mean \pm 1 s.d. over 10 networks.

green), only after cue offset but for most of the delay period for the just-in-time cost (Fig. 5c and Extended Data Fig. 6a, red), or only shortly before go time for the after-go-time cost (Fig. 5c and Extended Data Fig. 7b, blue). We then further analyzed the dynamics with which these networks achieved competent performance. The cue-delay network showed signatures of attractor dynamics with simple pattern completion (Extended Data Fig. 6d): full cross-temporal decoding was high between the cue and delay epochs (Fig. $5 d$, left), and although there was a cross-over of our overlap measures, critically, neural activities were already aligned well above chance with the most persistent modes immediately following cue onset (Fig. 5e, left). We also found similar dynamics for networks that optimize a 'full-delay' cost in which cue information must be stably maintained immediately after cue offset (rather than after cue onset as in the cue-delay networks; Extended Data Fig. 8, Methods). At the other extreme, the after-go-time network did not appear to make use of attractor dynamics at all and instead generated largely sequential activities (akin to the dynamics of a purely a feedforward network, Extended Data Fig. 5c): cross-temporal decoding remained poor even between most time points within the delay period (Fig. 5d, right; this effect was particularly strong for a fixed delay task, Extended Data Fig. 7c, right), and the overlap with the most persistent modes never significantly exceeded that with the most amplifying modes (Fig. 5e, right). Thus, the network dynamics trained for both of these classical cost functions were different from that predicted by attractor networks with optimal information loading (Fig. 3) and they were also different from our experimental recordings (Fig. 4b,c,f). Finally, in contrast to both classical training costs, just-intime networks showed attractor dynamics (Extended Data Fig. 6d) with optimal information loading: crosstemporal decoding was poor between early and late periods of a trial, but high during the late delay period (Fig. 5d, middle; Extended Data Fig. 6b), and the overlap of neural activities with the most amplifying and persistent modes showed the characteristic cross-over that we predicted theoretically and found experimentally (Fig. 5e, middle). These results suggest that optimal information loading in attractor networks emerges naturally, without explicit requirements, as a dynamical strategy for networks optimizing a just-in-time cost.

\section{Discussion}

While attractor networks have been proposed to underlie a number of core cognitive functions $^{4-6,8,25-27,30,30,44,45,51,68-70}$, prominently including working memory ${ }^{5-12}$, their operation was almost exclusively analyzed in terms of how they support information maintenance $4,5,7,8,12,25,26,44,71,72$. We instead studied information loading in attractor networks and showed that optimal information loading provides a normative account of the widely observed and puzzling phenomenon of dynamic coding ${ }^{13-16,32}$. Our 
theory also revealed a novel, theoretically-grounded aspect of dynamic coding: not only should neural activities during the cue and early delay period be orthogonal to those during the late delay period, but they should be orthogonal in the specific directions that are aligned with the (locally) most amplifying directions. We found strong evidence for these predictions of optimal information loading in PFC during a memoryguided saccade task. We predict that these results should also generalise to more cognitively demanding working memory tasks in which, unlike in the simple memory-guided saccade task we studied here, the correct response is unknown during the delay period, thus requiring the maintenance of stimulus information before a response can be prepared ${ }^{13,14,17,20,41}$. Indeed, strongly dynamic population activity, similar to those that we identified here, have been observed in such tasks ${ }^{13-16,20,41}$.

Our focus has been on identifying a fundamental mechanism, attractor dynamics with optimal information loading, that underlies working memory in neural circuits. There are different ways in which this mechanism can be realized in specific, detailed neural network models. We have shown examples for this in networks with both linear and nonlinear dynamics, which both reproduced the features of the experimental data that were most puzzling (dynamic coding) as well as those that were diagnostic of this core mechanism (overlap of neural activity with persistent and amplifying modes). Nevertheless, there are also aspects of the data that none of these specific models reproduced accurately. First, the overlap with the most amplifying directions became significantly lower than chance over time in the data. This suggests that PFC circuits may be more mathematically 'non-normal' (i.e. include stronger feedforward loops ${ }^{18,33}$, or excitatoryinhibitory interactions ${ }^{53}$ ) than the networks with randomly chosen or initialised weights we used here ${ }^{53,56}$. Second, the time evolution of the overlaps with the most persistent and most amplifying modes seemed to obey different time constants, with the persistent overlap evolving substantially slower than the amplifying overlap. This may be a result of high dimensional, graded dynamical transitions between multiple amplifying and persistent modes rather than the less complex dynamical transitions that we observed in our models.

Our theory unifies previous, seemingly conflicting models of working memory maintenance that typically either use attractor dynamics ${ }^{7,8,11}$ or rely on sequential activities often generated by non-normal dynamics ${ }^{18,33,34,61}$. We found that although both classes of models can capture select aspects of neural data (e.g. sequential models can capture early delay activity whereas attractors are better suited to capturing late delay activity), no model could capture the experimentally observed rich combination of sequential and persistent dynamics ${ }^{17}$ (see also ${ }^{66}$ ). We showed that optimal information loading in attractor models with realistic, unconstrained connectivity, leads to the spe- cific combination of sequential and persistent dynamics that has been observed in experiments.

There have been multiple mechanisms proposed to account for some of the features of the data that seem to be at odds with basic attractor network dynamics, most prominently dynamic coding ${ }^{13,14}$. These hypothetical mechanisms include short-term plasticity $20,21,35,36$, specific changes in the strength of input and recurrent connections ${ }^{37,38}$, and separate stimulus- and delay-responsive cells ${ }^{16,29}$. We showed that the core phenomenon of dynamic coding emerges naturally, without any of these additional mechanisms, from the same ultimate principle that explains persistent activities (robust memory maintenance implemented by attractor dynamics). Moreover, the high initial overlap with the most amplifying modes, which was a core prediction of our theory confirmed by the data, is not specifically predicted by any of these alternative mechanisms. Nevertheless, these mechanisms are not mutually exclusive with ours. In fact, they might help explain the more nuanced aspects of the data that our specific network implementations did not capture (see above; for example the structured orthogonality described by Ref. 39), as well as aspects of the data that lie outside the scope of our theory (e.g. activity silent information maintenance during inter-trial intervals $\left.{ }^{60}\right)$.

The importance of optimal initialization of network dynamics has been recognized previously in the context of movement preparation, when the transients following initialisation are themselves thought to encode relevant (movement) information ${ }^{53,56,73,74}$. Our work suggests that optimal initialization is a more general principle of the dynamics of cortical circuits, extending beyond the motor cortex. In particular, our results demonstrate the importance of optimal initialization even when the transients following initialization themselves may be irrelevant ${ }^{23}$, as information is ultimately maintained by stable attractor states.

\section{References}

1. Manes, F. et al. Decision-making processes following damage to the prefrontal cortex. Brain 125, 624-639 (2002).

2. Baddeley, A. Working memory: Looking back and looking forward. Nature Reviews Neuroscience 4, 829-839 (2003).

3. Hebb, D. O. The organization of behavior: a neuropsychological theory (Wiley, 1949).

4. Wang, X.-j. Synaptic reverberation underlying mnemonic persistent activity. Trends in Neurosciences 24, 455-463 (2001).

5. Brody, C. D., Romo, R. \& Kepecs, A. Basic mechanisms for graded persistent activity: Discrete attractors, continuous attractors, and dynamic representations. Current Opinion in Neurobiology 13, 204-211 (2003).

6. Durstewitz, D., Seamans, J. K. \& Sejnowski, T. J. Neurocomputational Models of Working Memory. Nature Neuroscience 3, 1184-1191 (2000). 
bioRxiv preprint doi: https://doi.org/10.1101/2021.11.16.468360; this version posted December 17, 2021. The copyright holder for this preprint (which was not certified by peer review) is the author/funder, who has granted bioRxiv a license to display the preprint in perpetuity. It is made available under aCC-BY 4.0 International license.

7. Wimmer, K., Nykamp, D. Q., Constantinidis, C. \& Compte, A. Bump attractor dynamics in prefrontal cortex explains behavioral precision in spatial working memory. Nature Neuroscience 17, 431-439 (2014).

8. Amit, D. J. \& Brunel, N. Model of global spontaneous activity and local structured activity during delay periods in the cerebral cortex. Cerebral Cortex 7, 237-252 (1997).

9. Brunel, N. Dynamics and Plasticity of Stimulusselective Persistent Activity in Cortical Network Models. Cerebral Cortex 13, 1151-1161 (2003).

10. Machens, C. K., Romo, R. \& Brody, C. D. Flexible control of mutual inhibition: A neural model of twointerval discrimination. Science 307, 1121-1124 (2005).

11. Murray, J. D. et al. Stable population coding for working memory coexists with heterogeneous neural dynamics in prefrontal cortex. Proceedings of the National Academy of Sciences 114, 394399 (2017).

12. Druckmann, S. \& Chklovskii, D. B. Neuronal circuits underlying persistent representations despite time varying activity. Current Biology 22, 2095-2103 (2012).

13. Meyers, E. M., Freedman, D. J., Kreiman, G., Miller, E. K. \& Poggio, T. Dynamic population coding of category information in inferior temporal and prefrontal cortex. Journal of Neurophysiology 100, 1407-1419 (2008).

14. Stokes, M. G. et al. Dynamic coding for cognitive control in prefrontal cortex. Neuron 78, 364-375 (2013).

15. Meyers, E. M. Dynamic population coding and its relationship to working memory. Journal of $\mathrm{Neu}$ rophysiology 120, 2260-2268 (2018).

16. Spaak, E., Watanabe, K., Funahashi, S. \& Stokes, M. G. Stable and Dynamic Coding for Working Memory in Primate Prefrontal Cortex. The Journal of Neuroscience 37, 6503-6516 (2017).

17. Lundqvist, M., Herman, P. \& Miller, E. K. Working Memory: Delay Activity, Yes! Persistent Activity? Maybe Not. The Journal of Neuroscience 38, 7013-7019 (2018).

18. Goldman, M. S. Memory without Feedback in a Neural Network. Neuron 61, 621-634 (2009).

19. Machens, C. K., Romo, R. \& Brody, C. D. Functional, but not anatomical, separation of "what" and "when" in prefrontal cortex. Journal of Neuroscience 30, 350-360 (2010).

20. Barak, O., Tsodyks, M. \& Romo, R. Neuronal population coding of parametric working memory. Journal of Neuroscience 30, 9424-9430 (2010).

21. Stokes, M. G. 'Activity-silent' working memory in prefrontal cortex: A dynamic coding framework. Trends in Cognitive Sciences 19, 394-405 (2015).

22. Barak, O., Sussillo, D., Romo, R., Tsodyks, M. \& Abbott, L. F. From fixed points to chaos: Three models of delayed discrimination. Progress in Neurobiology 103, 214-222 (2013).
23. Cueva, C. J. et al. Low-dimensional dynamics for working memory and time encoding. Proceedings of the National Academy of Sciences of the United States of America 117, 23021-23032 (2020).

24. Scott, B. B. et al. Fronto-parietal Cortical Circuits Encode Accumulated Evidence with a Diversity of Timescales. Neuron 95, 385-398 (2017).

25. Inagaki, H. K., Fontolan, L., Romani, S. \& Svoboda, K. Discrete attractor dynamics underlies persistent activity in the frontal cortex. Nature 566, 212-217 (2019).

26. Seung, H. S. How the brain keeps the eyes still. Proceedings of the National Academy of Sciences of the United States of America 93, 13339-13344 (1996).

27. Hopfield, J. J. Neural networks and physical systems with emergent collective computational abilities. Proceedings of the National Academy of Sciences of the United States of America 79, 25542558 (1982).

28. Chadwick, A. et al. Learning Shapes Cortical Dynamics to Enhance Integration of Relevant Sensory Input. bioRxiv 454726 (2021).

29. Goldman-Rakic, P. S. Cellular basis of working memory. Neuron 14, 477-485 (1995).

30. Mante, V., Sussillo, D., Shenoy, K. V. \& Newsome, W. T. Context-dependent computation by recurrent dynamics in prefrontal cortex. Nature 503, 78-84 (2013).

31. Sreenivasan, K. K., Curtis, C. E. \& D'Esposito, $M$. Revisiting the role of persistent neural activity during working memory. Trends in Cognitive Sciences 18, 82-89 (2014).

32. Cavanagh, S. E., Towers, J. P., Wallis, J. D., Hunt, L. T. \& Kennerley, S. W. Reconciling persistent and dynamic hypotheses of working memory coding in prefrontal cortex. Nature Communications 9, 3498 (2018).

33. Ganguli, S., Huh, D. \& Sompolinsky, H. Memory traces in dynamical systems. Proceedings of the National Academy of Sciences of the United States of America 105, 18970-18975 (2008).

34. Rajan, K. et al. Recurrent Network Models of Sequence Generation and Memory Recurrent Network Models. Neuron 90, 128-142 (2016).

35. Mongillo, G., Barak, O. \& Tsodyks, M. Synaptic Theory of Working Memory. Science 319, 15431546 (2008).

36. Masse, N. Y., Yang, G. R., Song, H. F., Wang, X. J. \& Freedman, D. J. Circuit mechanisms for the maintenance and manipulation of information in working memory. Nature Neuroscience 22, 11591167 (2019).

37. Parthasarathy, A. et al. Time-Invariant Working Memory Representations in the Presence of Code-Morphing in the Lateral Prefrontal Cortex. Nature Communications 10, 4995 (2019).

38. Bouchacourt, F. \& Buschman, T. J. A Flexible Model of Working Memory. Neuron 103, 147160.e8 (2019).

39. Libby, A. \& Buschman, T. J. Rotational dynamics reduce interference between sensory and mem- 
bioRxiv preprint doi: https://doi.org/10.1101/2021.11.16.468360; this version posted December 17, 2021. The copyright holder for this preprint (which was not certified by peer review) is the author/funder, who has granted bioRxiv a license to display the preprint in perpetuity. It is made available under aCC-BY 4.0 International license.

ory representations. Nature Neuroscience 24, 715-727 (2021).

40. Sigala, N., Kusunoki, M., Nimmo-Smith, I., Gaffan, D. \& Duncan, J. Hierarchical coding for sequential task events in the monkey prefrontal cortex. Proceedings of the National Academy of Sciences of the United States of America 105, 11969-11974 (2008).

41. Brody, C. D., Hernández, A., Zainos, A. \& Romo, R. Timing and Neural Encoding of Somatosensory Parametric Working Memory in Macaque Prefrontal Cortex. Cerebral Cortex 13, 11961207 (2003).

42. Ehrlich, D. B. \& Murray, J. D. Geometry of neural computation unifies working memory and planning. bioRxiv 2021.02.01.429156 (2021).

43. Machens, C. K. \& Brody, C. D. Design of continuous attractor networks with monotonic tuning using a symmetry principle. Neural Computation 20, 452-485 (2008).

44. Cannon, S. C., Robinson, D. A. \& Shamma, S. A proposed neural network for the integrator of the oculomotor system. Biological Cybernetics 49, 127-136 (1983).

45. Amit, D. J. Modeling brain function: The world of attractor neural networks (Cambridge University Press, 1992).

46. Compte, A., Brunel, N., Goldman-Rakic, P. S. \& Wang, X. J. Synaptic mechanisms and network dynamics underlying spatial working memory in a cortical network model. Cerebral Cortex 10, 910923 (2000).

47. Hopfield, J. J. Neurons with graded response have collective computational properties like those of two-state neurons. Proceedings of the National Academy of Sciences of the United States of America 81, 3088-3092 (1984).

48. Dayan, P. \& Abbott, L. Theoretical Neuroscience: Computational and Mathematical Modeling of Neural Systems (MIT Press, 2001).

49. Hennequin, G., Vogels, T. P. \& Gerstner, W. Nonnormal amplification in random balanced neuronal networks. Physical Review E - Statistical, Nonlinear, and Soft Matter Physics 86, 1-12 (2012).

50. Murphy, B. K. \& Miller, K. D. Balanced Amplification: A New Mechanism of Selective Amplification of Neural Activity Patterns. Neuron 61, 635-648 (2009).

51. Wong, K. F. \& Wang, X. J. A recurrent network mechanism of time integration in perceptual decisions. Journal of Neuroscience 26, 1314-1328 (2006).

52. Ganguli, S. et al. One-Dimensional Dynamics of Attention and Decision Making in LIP. Neuron 58, 15-25 (2008).

53. Hennequin, G., Vogels, T. P. \& Gerstner, W. Optimal control of transient dynamics in balanced networks supports generation of complex movements. Neuron 82, 1394-1406 (2014).

54. Kao, T. C. \& Hennequin, G. Neuroscience out of control: control-theoretic perspectives on neural circuit dynamics. Current Opinion in Neurobiology
58, 122-129 (2019).

55. Stroud, J. P., Porter, M. A., Hennequin, G. \& Vogels, T. P. Motor primitives in space and time via targeted gain modulation in cortical networks. $\mathrm{Na}$ ture Neuroscience 21, 1774-1783 (2018).

56. Kao, T. C., Sadabadi, M. S. \& Hennequin, G. Optimal anticipatory control as a theory of motor preparation: A thalamo-cortical circuit model. Neuron 109, 1567-1581 (2021).

57. Trefethen, L. N. Spectra and Pseudospectra (Princeton University Press, 1999).

58. Funahashi, S., Bruce, C. J. \& Goldman-Rakic, P. S. Mnemonic coding of visual space in the monkey's dorsolateral prefrontal cortex. Journal of Neurophysiology 61, 331-349 (1989).

59. Parthasarathy, A. et al. Mixed selectivity morphs population codes in prefrontal cortex. Nature Neuroscience 20, 1770-1779 (2017).

60. Barbosa, J. et al. Interplay between persistent activity and activity-silent dynamics in the prefrontal cortex underlies serial biases in working memory. Nature Neuroscience 23, 16-18 (2020).

61. Sompolinsky, H., Crisanti, A. \& Sommers, H. J. Chaos in random neural networks. Physical Review Letters 61, 259-262 (1988).

62. Maass, W., Natschläger, T. \& Markram, H. Realtime computing without stable states: A new framework for neural computation based on perturbations. Neural Computation 14, 2531-2560 (2002).

63. Enel, P., Procyk, E., Quilodran, R. \& Dominey, P. F. Reservoir Computing Properties of Neural Dynamics in Prefrontal Cortex. PLoS Computational Biology 12, 1-35 (2016).

64. Elsayed, G. F., Lara, A. H., Kaufman, M. T., Churchland, M. M. \& Cunningham, J. P. Reorganization between preparatory and movement population responses in motor cortex. Nature Communications 7, 13239 (2016).

65. Yang, G. R., Joglekar, M. R., Song, H. F., Newsome, W. T. \& Wang, X.-J. Task representations in neural networks trained to perform many cognitive tasks. Nature Neuroscience 22, 297-306 (2019).

66. Orhan, A. E. \& Ma, W. J. A diverse range of factors affect the nature of neural representations underlying short-term memory. Nature Neuroscience 22, 275-283 (2019).

67. Song, H. F., Yang, G. R. \& Wang, X. J. Training Excitatory-Inhibitory Recurrent Neural Networks for Cognitive Tasks: A Simple and Flexible Framework. PLoS Computational Biology 12, 1-30 (2016).

68. Rolls, E. T. An attractor network in the hippocampus: Theory and neurophysiology. Learning and Memory 14, 714-731 (2007).

69. Burak, Y. \& Fiete, I. R. Accurate path integration in continuous attractor network models of grid cells. PLoS Computational Biology 5, e1000291 (2009).

70. Kim, S. S., Rouault, H., Druckmann, S. \& Jayaraman, V. Ring attractor dynamics in the Drosophila 
central brain. Science 356, 849-853 (2017).

71. Burak, Y. \& Fiete, I. R. Fundamental limits on persistent activity in networks of noisy neurons. Proceedings of the National Academy of Sciences of the United States of America 109, 17645-17650 (2012).

72. Renart, A., Song, P. \& Wang, X. J. Robust spatial working memory through homeostatic synaptic scaling in heterogeneous cortical networks. Neuron 38, 473-485 (2003).

73. Churchland, M. M. et al. Neural population dynamics during reaching. Nature 487, 51-6 (2012).

74. Churchland, M. M., Cunningham, J. P., Kaufman, M. T., Ryu, S. I. \& Shenoy, K. V. Cortical Preparatory Activity: Representation of Movement or First Cog in a Dynamical Machine? Neuron 68, 387400 (2010).

\section{Acknowledgements}

This work was funded by the Wellcome Trust (Investigator Award in Science 212262/Z/18/Z to M.L. and Sir Henry Wellcome Postdoctoral Fellowship 215909/Z/19/Z to J.S.), the Human Frontiers Science Programme (Research Grant RGP0044/2018 to M.L.), the Biotechnology and Biological Sciences Research Council (award BB/M010732/1 to M.G.S.), the James S. McDonnell Foundation (award 220020405 to M.G.S.), the Japan Society for the Promotion of Science (JP18K03197 and JP21K03141 to K.W., $18 \mathrm{H} 05380$ to T.S.), and the Japan Science and Technology Agency (CREST JPMJCR186 to T.S.). For the purpose of open access, the authors have applied a CC-BY public copyright licence to any author accepted manuscript version arising from this submission. We thank Flavia Mancini, John Duncan, Guillaume Hennequin, and Yashar Ahmadian for useful feedback and detailed comments on the manuscript.

\section{Author Contributions}

J.P.S., M.L., and M.G.S. conceived the study. K.W. performed all experimental recordings, T.S. assembled the neural recording system, and K.W. and T.S. performed data pre-processing. J.P.S. and M.L. developed the theoretical framework, performed analytical derivations, and wrote the first draft of the manuscript. J.P.S. performed all numerical simulations, analysed the data, and produced the figures. J.P.S, M.L., and M.G.S. interpreted the results. All authors revised the final manuscript.

Competing Interests statement. The authors declare no competing interests.

Reporting Summary. Further information on research design is available in the Life Sciences Reporting Summary linked to this article.

Randomization. No new experimental data was gathered for this paper. There is no group allocation in this study. Trial types were randomly determined by a computer program.

Blinding. As data collection had been performed well before our theory of optimal information loading was developed and our corresponding analyses were performed, it was effectively blind to the purposes of our study. Data analysis was not performed blind to the conditions of the experiments.

Data exclusion. As described below (Preprocessing), 1 neuron from monkey K's dataset was removed from all analyses because it was recorded in fewer than 10 trials for at least one stimulus cue condition. As also described below (Pre-processing), randomly generated linear networks with unconstrained connectivity in Fig. 3 were excluded if the inner product between the most amplifying mode and persistent mode was greater than 0.05 (i.e. we only kept networks that were relatively mathematically non-normal).

Data availability. All experimental data will be made available in the following repository upon peerreviewed publication: https://github.com/jakepstroud.

Code availability. All code was custom written in Python using NumPy, SciPy, Matplotlib, Scikit-learn, and Tensorflow libraries. All code will be made available in the following repository upon peer-reviewed publication: https://github.com/jakepstroud. 
bioRxiv preprint doi: https://doi.org/10.1101/2021.11.16.468360; this version posted December 17, 2021. The copyright holder for this preprint (which was not certified by peer review) is the author/funder, who has granted bioRxiv a license to display the preprint in perpetuity. It is made available under aCC-BY 4.0 International license.

\section{Methods}

\section{Experimental materials and methods}

Experimental methods largely followed those used in our previous publications ${ }^{16,75,76}$, and are briefly summarized below.

\section{Subjects and apparatus}

We used two female macaques (monkey T, Macaca mulatta, $5 \mathrm{~kg}$; monkey $\mathrm{K}$, Macaca fuscata, $8 \mathrm{~kg}$ ). Both monkeys were housed individually. The light/dark cycle was 12/12 hr. (light, from 8:30 a.m. to 8:30 p.m.). The monkeys sat quietly in a primate chair in a dark, sound-attenuated shield room. During both training and neural recording sessions, we restrained the monkeys' head movement non-invasively using a thermoplastic head cap as described in ${ }^{77}$. This head cap is made of a standard thermoplastic splint material (MT-APU, $3.2 \mathrm{~mm}$ thick, CIVCO Radiotherapy, IA., USA), and was molded out so that it conformed to the contours of the animals' scalp, cheek bone, and occipital ridge. Visual stimuli were presented on a 17 inch TFT monitor placed $50 \mathrm{~cm}$ from the monkeys' eyes. Eye movements were sampled at $120 \mathrm{~Hz}$ using an infrared eye tracking system (ETL-200, ISCAN, MA.). Eye fixation was controlled within a $6.5^{\circ}$ imaginary square window. TEMPO software (Reflective Computing, WA.) was used to control behavioral tasks. All experimental procedures were approved by the Animal Research Committee at the Graduate School of Frontier Biosciences, Osaka University, Japan and were in full compliance with the guidelines of the National BioResource Project "Japanese Macaques". Experimental work performed in non-human primates that was not funded by Wellcome may not adhere to the principles outlined in the NC3Rs guidance on Non-human Primate Accommodation, Care and Use.

\section{Behavioral task}

The monkeys were trained on a memory-guided saccade task requiring them to remember the location of a visual stimulus cue on a screen and to make a correct eye movement after a delay period (Fig. 1a). Specifically, this task required monkeys to fixate on a central ring for a period of 2.6-7.4 s followed by a stimulus cue (a white square) appearing in one of six pre-determined locations for $0.25 \mathrm{~s}$. After a variable delay period of 1.4-7.5 s, the fixation ring was replaced by placeholders at all six possible stimulus cue locations (go cue). Monkeys were required to make a saccade within $0.5 \mathrm{~s}$ to the placeholder where the original stimulus cue was presented and maintain their gaze for $0.25 \mathrm{~s}$ for monkey $\mathrm{T}$ and either $0.25 \mathrm{~s}$ or $0.6 \mathrm{~s}$ for monkey $\mathrm{K}$ (these two gaze maintenance times were switched in different blocks for monkey $\mathrm{K}$ ) to receive a juice reward. The monkeys were extensively trained, with close to perfect performance (monkey T, 96.1\%; monkey K, 96.3\%, mean across sessions). Fixation break errors were excluded from the calculation of percent correct rate.

\section{Recordings}

After training was completed, we conducted an aseptic surgery under general anesthesia. We stereotypically implanted a plastic recording chamber on the lateral surface of the prefrontal cortex, under the guidance of structural MRI images (Extended Data Fig. 9). In monkey T, we implanted a cylindrical chamber (RC-T-S-P, internal diameter $12.7 \mathrm{~mm}$, Gray Matter Research, MT.) in the right hemisphere (AP = 33, ML = 14.5; $A P$, anteriorposterior; ML, medio-lateral). A 32-channel semi-chronic microdrive system (SC-32, Gray Matter Research) was mounted inside this chamber. In monkey $\mathrm{K}$, we implanted a cuboid chamber (width $12 \mathrm{~mm}$, depth $16 \mathrm{~mm}$, height, $15 \mathrm{~mm}$, S-company Itd., Tokyo, Japan) over the principal sulcus in the left hemisphere.

We collected neural data in a total of 48 daily sessions (21 in monkey T; 27 in monkey K). In monkey T, we used the 32-ch microdrive (SC-32) that housed 32 single-contact tungsten electrodes with inter-electrode spacing of $1.5 \mathrm{~mm}$. In monkey K, we used a 32-ch linear microelectrode array (Plexon U-Probe, Plexon, TX.) with an interelectrode spacing of $150 \mu \mathrm{m}$ along a single shaft. We positioned the U-Probe by using a custom-made grid (width $12 \mathrm{~mm}$, depth $16 \mathrm{~mm}$, height, $10 \mathrm{~mm}$ ) which had a total of 165 holes with $1 \mathrm{~mm}$ spacing. We advanced the U-Probe by a custom-made hydraulic microdrive (S-company Itd.).

Raw extracellular neural signals were amplified and recorded in reference to a titanium bone screw at the vertex (in monkey T) or the shaft of the linear array (monkey K) using a neural signal amplifier RZ2 Bioamp Processor (Tucker-Davis Technologies, FL.). Behavioral data (task-event information and eye-movement information) were also sent to the RZ2 Bioamp. Neural data acquisition was performed at a sampling frequency of $24414.08 \mathrm{~Hz}$, and behavioral data acquisition at $1017.25 \mathrm{~Hz}$. For analysis of spiking activity, the raw neural signal was filtered (300 Hz to $6 \mathrm{kHz}$ ) for offline sorting (Offline Sorter, Plexon). In monkey T, approximately three hours before each recording session, we took the monkey to the testing room and advanced each electrode in the SC-32 
bioRxiv preprint doi: https://doi.org/10.1101/2021.11.16.468360; this version posted December 17, 2021. The copyright holder for this preprint (which was not certified by peer review) is the author/funder, who has granted bioRxiv a license to display the preprint in perpetuity. It is made available under aCC-BY 4.0 International license.

by a minimum of $62.5 \mu \mathrm{m}$ in order to ensure recording of new neurons. We then put the monkey back in the home cage until we brought it out again for the recording session. In monkey $\mathrm{K}$, we adopted the method of the U-Probe insertion reported in ${ }^{78}$. We first punctuated the dura using a guide tube (a shortened 23 gauge needle), and inserted the U-Probe array slowly, usually with a step of $500 \mu \mathrm{m}$. We kept monitoring electrocardiogram (pulsatory fluctuation) on superficial electrodes to identify the point of cortical entry. We usually left 3-5 superficial channels outside the cortex. After array insertion, we waited 1-1.5 hours until the recorded single-unit and multiunit activities indicated that the electrode array was stably positioned in the cortex. While waiting, the monkey watched nature and animal video clips and received a small snack on a monkey chair.

In monkey $T$ only, to determine location of the frontal eye field (FEF), and confirm that our recording area was outside it, intracortical microstimulations (22 biphasic pulses, $0.2 \mathrm{~ms}$ duration at $333 \mathrm{~Hz}, \leq 150 \mu \mathrm{A}$ ) were applied through microelectrodes. When eye movements were elicited below $50 \mu \mathrm{A}$, the site was considered to be in the low-threshold FEF. In monkey T, our recording area did not include the low-threshold FEF.

\section{Pre-processing}

We excluded neurons that were recorded in fewer than 10 trials for any cue condition. For each monkey, we pooled neurons from all recording sessions to create pseudopopulations of 438 neurons for Monkey K (after we removed 1 neuron from monkey K's dataset due to an insufficient number of trials) and 625 neurons for Monkey $\mathrm{T}$ (no neurons were removed from monkey T's dataset). To compute neural firing rates, we convolved spike trains with a Gaussian kernel with a standard deviation of $25 \mathrm{~ms}$. Trial-averaged trajectories of time-varying mean firing rates were computed separately for each neuron and each cue condition. For analysis methods that used crossvalidation (see below), we split trials into separate train and test sets with a 1:1 train:test ratio, and computed trial-averaged trajectories for each training and test set (using 1:1 splits). For non-cross validated analyses, we either computed trial averages based on all the data, or on a subset of the data (see below). We aligned neural activity to either stimulus or go cue onset (see also below in Analysis methods) and shifted activity by -50 ms to allow for the delay in time for information about these cues to enter PFC. For consistency with our simulations (see below), we subsampled neural firing rates at a 1-ms time resolution.

\section{Neural network models: overview}

All our simulated networks (Figs. 1-3 and 5 and Extended Data Figs. 1, 3, 4 and 6-8) evolved according to a canonical model of stochastic recurrent neural circuit dynamics ${ }^{67,79}$ :

with

$$
\begin{aligned}
\tau \frac{\mathrm{d} \mathbf{x}^{(c)}(t)}{\mathrm{d} t} & =-\mathbf{x}^{(c)}(t)+\mathbf{W} \mathbf{r}^{(c)}(t)+m_{\mathrm{h}}(t) \mathbf{h}^{(c)}+m_{\mathrm{g}}(t) \mathbf{g}+\mathbf{b}+\sigma \boldsymbol{\eta}^{(c)}(t) \\
\mathbf{r}^{(c)}(t) & =\mathbf{f}\left(\mathbf{x}^{(c)}(t)\right)
\end{aligned}
$$

where $\mathbf{x}^{(c)}(t)=\left(x_{1}^{(c)}(t), \ldots, x_{N}^{(c)}(t)\right)^{\top}$ corresponds to the vector of (unitless) 'subthreshold', i.e. coarse-grained (lowpass filtered), trial-averaged raw somatic membrane potentials of the $N$ neurons of the network ${ }^{79}$ in cue condition $c=1, \ldots, C$ (initialised at $\mathbf{x}^{(c)}\left(t_{0}\right)$ at the beginning of the simulation $t_{0}$, which could be at or before stimulus onset at $t=0), \mathbf{r}^{(c)}(t)$ is their momentary (trial-averaged) firing rates, with $\mathbf{f}(\mathbf{x})$ being the activation function that converts membrane potentials to firing rates, $\tau$ is the membrane time constant, $\mathbf{W}$ is the recurrent weight matrix (shown e.g. in Fig. $1 \mathrm{~b}$ and $\mathbf{g}), \mathbf{h}^{(c)}$ is the input given to the network depending on the stimulus cue, $\mathbf{g}$ is the stimulus-cueindependent go cue that occurs at the go time $t_{\mathrm{go}}, m_{\mathrm{h}}(t)$ and $m_{\mathrm{g}}(t)$ are box car 'masking' kernels such that the stimulus and go cues are only effective within a limited period at the beginning and end of the trial, respectively, $\mathbf{b}$ is a cue-independent bias, $\sigma$ is the standard deviation of the noise process, and $\boldsymbol{\eta}^{(c)}(t)$ is a sample from a standard (mean 0 and variance 1) Gaussian white (temporally and spatially) noise process. Note that $\eta^{(c)}(t)$ represents the effective noise corrupting trial-averaged trajectories (rather than the noise corrupting individual trials).

Networks shown in different figures corresponded to different special cases of Eqs. 1 and 2 (see Table 1). Specifically, for linear networks (Figs. 2 and 3, Fig. 4d,e,f, Fig. 5e, Extended Data Fig. 3d, Extended Data Fig. 4, and Extended Data Fig. 10f,g) $\mathbf{f}(\mathbf{x})=\mathbf{x}$ was the identity function ${ }^{11,12,18,26,33,44}$. For nonlinear networks (Figs. 1 and 5 and Extended Data Figs. 1 and 6-8) $f_{i}(\mathbf{x})=\left[x_{i}\right]_{+}$was the rectified linear (ReLU) activation function applied element-wise ${ }^{56,66,67}$. Given that the focus of our study was optimal information loading, stimulus inputs were either optimized numerically (Fig. 1, Fig. 5, Extended Data Fig. 1, Extended Data Fig. 4c,d, and Extended Data Figs. 6-8), or set to analytically computed values as dictated by our mathematical analysis (Figs. 2 and 3 and Extended Data Fig. 4a,b), or as a baseline, set to random (or quasi-random, see below) values (Figs. 2 and 3 and Extended Data Fig. 4a,b). For networks used to study the effects of instantaneous initial conditions (Figs. 1 and 2 and Extended Data Figs. 1, 3 and 4), the stimulus masking kernel was zero and instead the initial condition was set to the stimulus input; for other networks (Fig. 3, Fig. 4e-f, Fig. 5 and Extended Data Figs. 6-8) the stimulus masking kernel was a boxcar between 0 and $0.25 \mathrm{~s}$ and the initial conditions were either set to zero (Fig. 3, Fig. 4e-f) or sampled randomly around zero (Fig. 5 and Extended Data Figs. 6-8). For functionally trained 


\begin{tabular}{|c|c|c|c|c|c|c|}
\hline Symbol & Fig. 1 & Fig. $2 a-c$ & Fig. $2 \mathrm{~d}-\mathrm{e}$ & Fig. 3 & Units & Description \\
\hline $\mathrm{N}$ & 50 & 2 & 1000 & 100 & - & number of neurons \\
\hline$\tau$ & 0.05 & 0.05 & 0.05 & 0.05 & $\mathrm{~s}$ & membrane time constant \\
\hline$t_{0}$ & 0 & 0 & 0 & -0.5 & $\mathrm{~S}$ & simulation start time \\
\hline $\mathbf{x}^{(c)}\left(t_{0}\right)$ & $\mathbf{h}^{(c)}$ & $\mathbf{h}^{(c)}$ & $\mathbf{h}^{(c)}$ & $\mathbf{0}$ & - & initial condition \\
\hline $\mathbf{f}(\cdot)$ & nonlinear $^{a}$ & linear ${ }^{a}$ & linear $^{a}$ & linear ${ }^{a}$ & $\mathrm{~Hz}$ & neural activation function \\
\hline W & optimized $^{b}$ & $\operatorname{set}^{c}$ & $\stackrel{\text { iid. }}{\sim} \mathcal{N}\left(0, \frac{1}{N}\right)^{d}$ & $\stackrel{\text { iid. }}{\sim} \mathcal{N}\left(0, \frac{1}{N}\right)^{d}$ & $\mathrm{~s}$ & weight matrix \\
\hline$C$ & 6 & 1 & 1 & 2 & - & number of stimuli \\
\hline $\mathbf{h}^{(c)}$ & optimized $^{b}$ & $\operatorname{set}^{e}$ & $\operatorname{set}^{b}$ & $\operatorname{set}^{b}$ & - & stimulus input \\
\hline $\mathbf{g}$ & - & - & - & - & - & go cue \\
\hline$m_{\mathrm{h}}(t)$ & 0 & 0 & 0 & $K(0,0.25)^{f}$ & - & stimulus masking kernel \\
\hline$m_{\mathrm{g}}(t)$ & 0 & 0 & 0 & 0 & - & go cue masking kernel \\
\hline b & optimized $^{b}$ & $\mathbf{0}$ & $\mathbf{0}$ & $\mathbf{0}$ & - & cue-independent bias \\
\hline$\sigma$ & 0.05 & 0 & 0 & variable $^{b}$ & - & noise standard deviation \\
\hline Symbol & Fig. $4 \mathrm{e}-\mathrm{f}$ & Fig. $5 a-d$ & Fig. 5e & $\begin{array}{ll}\text { Extended } & \text { Data } \\
\text { Fig. } 1 & \end{array}$ & Units & Description \\
\hline $\mathrm{N}$ & 20 & 50 & 20 & 50 & - & number of neurons \\
\hline$\tau$ & 0.05 & 0.05 & 0.05 & 0.05 & $\mathrm{~s}$ & membrane time constant \\
\hline$t_{0}$ & 0 & -0.5 & 0 & 0 & $\mathrm{~s}$ & simulation start time \\
\hline $\mathbf{x}^{(c)}\left(t_{0}\right)$ & $\mathbf{0}$ & $\stackrel{\text { iid. }}{\sim} \mathcal{N}\left(0, \frac{1}{N}\right)$ & $\mathbf{0}$ & $\mathbf{h}^{(c)}$ & - & initial condition \\
\hline$f(\cdot)$ & linear $^{a}$ & nonlinear $^{a}$ & linear $^{a}$ & nonlinear $^{a}$ & $\mathrm{~Hz}$ & neural activation function \\
\hline W & $\mathrm{fit}^{b}$ & optimized $^{b}$ & $\mathrm{fit}^{b}$ & optimized $^{b}$ & $\mathrm{~s}$ & weight matrix \\
\hline$C$ & 6 & 6 & 6 & 6 & - & number of stimuli \\
\hline $\mathbf{h}^{(c)}$ & $\mathrm{fit}^{b}$ & optimized $^{b}$ & $\mathrm{fit}^{b}$ & optimized $^{b}$ & - & stimulus input \\
\hline g & - & $\sum_{c} \mathbf{h}^{(c)}$ & - & - & - & go cue \\
\hline$m_{\mathrm{h}}(t)$ & $K(0,0.25)^{f}$ & $K(0,0.25)^{f}$ & $K(0,0.25)^{f}$ & 0 & - & stimulus masking kernel \\
\hline$m_{\mathrm{g}}(t)$ & 0 & $K\left(t_{\mathrm{go}}, t_{\mathrm{go}}+0.5\right)^{f}$ & 0 & 0 & - & go cue masking kernel \\
\hline b & $\mathrm{fit}^{b}$ & optimized $^{b}$ & $\mathrm{fit}^{b}$ & optimized $^{b}$ & - & cue-independent bias \\
\hline$\sigma$ & 0 & 0.05 & 0 & 0 & - & noise standard deviation \\
\hline Symbol & $\begin{array}{l}\text { Extended } \\
\text { Data Fig. 3b }\end{array}$ & $\begin{array}{l}\text { Extended Data } \\
\text { Fig. 3c }\end{array}$ & $\begin{array}{l}\text { Extended Data } \\
\text { Fig. 3d }\end{array}$ & $\begin{array}{l}\text { Extended Data } \\
\text { Fig. 4a,b }\end{array}$ & Units & Description \\
\hline $\mathrm{N}$ & 50 & 50 & 20 & $10,100,1000$ & - & number of neurons \\
\hline$\tau$ & 0.05 & 0.05 & 0.05 & 0.05 & $\mathrm{~s}$ & membrane time constant \\
\hline$t_{0}$ & 0 & 0 & 0 & 0 & $\mathrm{~s}$ & simulation start time \\
\hline $\mathbf{x}^{(c)}\left(t_{0}\right)$ & $\mathbf{h}^{(c)}$ & $\mathbf{h}^{(c)}$ & $\mathbf{h}^{(c)}$ & $\mathbf{h}^{(c)}$ & - & initial condition \\
\hline $\mathbf{f}(\cdot)$ & linear $^{a}$ & nonlinear $^{a}$ & linear $^{a}$ & linear $^{a}$ & $\mathrm{~Hz}$ & neural activation function \\
\hline W & optimized $^{b}$ & optimized $^{b}$ & $\mathrm{fit}^{b}$ & $\stackrel{\text { iid. }}{\sim} \mathcal{N}\left(0, \frac{1}{N}\right)^{d}$ & $\mathrm{~s}$ & weight matrix \\
\hline C & 6 & 6 & 6 & 1 & - & number of stimuli \\
\hline $\mathbf{h}^{(c)}$ & optimized $^{b}$ & optimized $^{b}$ & $\mathrm{fit}^{b}$ & $\operatorname{set}^{b}$ & - & stimulus input \\
\hline $\mathbf{g}$ & - & - & - & - & - & go cue \\
\hline$m_{\mathrm{h}}(t)$ & 0 & 0 & 0 & 0 & - & stimulus masking kernel \\
\hline$m_{\mathrm{g}}(t)$ & 0 & 0 & 0 & 0 & - & go cue masking kernel \\
\hline b & optimized $^{b}$ & optimized $^{b}$ & $\mathrm{fit}^{b}$ & $\mathbf{0}$ & - & cue-independent bias \\
\hline$\sigma$ & 0 & 0.05 & 0 & 0 & - & noise standard deviation \\
\hline
\end{tabular}

Table 1 |Continued on text page. 
bioRxiv preprint doi: https://doi.org/10.1101/2021.11.16.468360; this version posted December 17, 2021. The copyright holder for this preprint (which was not certified by peer review) is the author/funder, who has granted bioRxiv a license to display the preprint in perpetuity. It is made available under aCC-BY 4.0 International license.

\begin{tabular}{|c|c|c|c|c|c|c|}
\hline Symbol & $\begin{array}{l}\text { Extended } \\
\text { Data Fig. 4c,d }\end{array}$ & $\begin{array}{ll}\text { Extended } & \text { Data } \\
\text { Figs. 6-8 } & \end{array}$ & $\begin{array}{l}\text { Extended } \\
\text { Data Fig. 10f }\end{array}$ & $\begin{array}{l}\text { Extended Data } \\
\text { Fig. } 10 \mathrm{~g}\end{array}$ & Units & Description \\
\hline $\mathrm{N}$ & 100 & 50 & 20 & 20 & - & number of neurons \\
\hline$\tau$ & 0.05 & 0.05 & 0.05 & 0.05 & $\mathrm{~s}$ & membrane time constant \\
\hline$t_{0}$ & 0 & -0.5 & 0 & $t_{\mathrm{go}}-1$ & $\mathrm{~s}$ & simulation start time \\
\hline $\mathbf{x}^{(c)}\left(t_{0}\right)$ & $\mathbf{h}^{(c)}$ & $\stackrel{\text { iid. }}{\sim} \mathcal{N}\left(0, \frac{1}{N}\right)$ & $\mathbf{0}$ & $\operatorname{set}^{b}$ & - & initial condition \\
\hline$f(\cdot)$ & linear $^{a}$ & nonlinear $^{a}$ & $\operatorname{linear}^{a}$ & linear $^{a}$ & $\mathrm{~Hz}$ & neural activation function \\
\hline W & $\stackrel{\text { iid. }}{\sim} \mathcal{N}\left(0, \frac{1}{N}\right)^{d}$ & optimized $^{b}$ & $\mathrm{fit}^{b}$ & $\mathrm{fit}^{b}$ & $\mathrm{~s}$ & weight matrix \\
\hline$C$ & & & 6 & 6 & - & number of stimuli \\
\hline $\mathbf{h}^{(c)}$ & optimized $^{b}$ & optimized $^{b}$ & $\mathrm{fit}^{b}$ & - & - & stimulus input \\
\hline g & - & $\sum_{c} \mathbf{h}^{(c)}$ & - & - & - & go cue \\
\hline$m_{\mathrm{h}}(t)$ & 0 & $K(0,0.25)^{f}$ & $K(0,0.25)^{f}$ & 0 & - & stimulus masking kernel \\
\hline$m_{\mathrm{g}}(t)$ & 0 & $K\left(t_{\mathrm{go}}, t_{\mathrm{go}}+0.5\right)^{f}$ & 0 & 0 & - & go cue masking kernel \\
\hline b & 0 & optimized $^{b}$ & $\mathrm{fit}^{b}$ & $\mathrm{fit}^{b}$ & - & cue-independent bias \\
\hline$\sigma$ & 0.05 & 0.05 & 0 & 0 & - & noise standard deviation \\
\hline
\end{tabular}

Table 1 | Parameters used in the simulations of our models.

a For nonlinear networks, $f_{i}(\mathbf{x})=\left[x_{i}\right]_{+}$was the rectified linear (ReLU) activation function. For linear networks $f_{i}(\mathbf{x})=x_{i}$. See also text.

${ }^{b}$ See text for details.

${ }^{c}$ For the symmetric network, we used $\left(\begin{array}{cc}0.375 & 0.625 \\ 0.625 & 0.375\end{array}\right)$; for the unconstrained network, we used $\left(\begin{array}{cc}-11.5 & 50 \\ 0 & 1\end{array}\right)$.

${ }^{d}$ For the symmetric networks, we enforced $\mathbf{W} \leftarrow \frac{1}{2}\left(\mathbf{W}+\mathbf{W}^{\top}\right)$. For all networks we also shifted the obtained weight matrix by the identity matrix multiplied by a constant so that the largest real part in the eigenvalues of $\mathbf{W}$ is exactly 1 (we rejected any $\mathbf{W}$ 's for which the eigenvalue with largest real part had an imaginary component). For Fig. 3, to provide a slightly better agreement between the model dynamics and the experimental recordings, we rejected any W's for which the inner product between the most amplifying mode and persistent mode was greater than 0.05 (i.e. we only kept W's that were relatively mathematically non-normal).

${ }^{e}$ We used 3 possible input directions (which all had a Euclidean norm of 1): inputs either aligned with the most persistent mode ( $\mathbf{x}^{\mathrm{p}}$ ), the most amplifying mode $\left(\mathbf{x}^{\mathrm{a}}\right)$, or a random direction $\left(\mathbf{x}^{\mathrm{r}}\right)$. For the symmetric model, $\mathbf{x}^{\mathrm{p}}=\mathbf{x}^{\mathrm{a}}=[0.707,0.707]^{\top}$ and we used $\mathbf{x}^{\mathrm{r}}=[0.98,0.18]^{\top}$. For the unconstrained model, $\mathbf{x}^{\mathrm{p}}=[1,0]^{\top}, \mathbf{x}^{\mathrm{a}}=[0.25,0.97]^{\top}$ and we used $\mathbf{x}^{\mathrm{r}}=[0.9,0.45]^{\top}$.

$f K\left(t_{1}, t_{2}\right)=\left\{\begin{array}{ll}1 & \text { if } t_{1} \leq t \leq t_{2} \mathrm{~S}, \\ 0 & \text { otherwise. }\end{array}\right.$ In the table, $t_{\mathrm{go}}$ refers to the timing of the go cue (see text).

networks (Fig. 5 and Extended Data Figs. 6-8), the go cue masking kernel $m_{\mathrm{g}}(t)$ was a boxcar starting at go cue onset, $t_{\mathrm{go}}$ (which could be fixed, Extended Data Fig. 7, or variable, Fig. 5 and Extended Data Figs. 6-8) and lasting for $0.5 \mathrm{~s}$, for all other networks it was set to 0 everywhere. The networks used to analyse the dynamics of information loading (Fig. 2 and Extended Data Fig. 4a,b) were deterministic by setting $\sigma=0$, all other networks used noisy dynamics (see Table 1). All models used a time constant of $\tau=50 \mathrm{~ms}$. We solved the dynamics of Eqs. 1 and 2 using a first-order Euler-Maruyama approximation between $t_{0}$ and the simulation end time, $t_{\max }$, with a discretization time step of $1 \mathrm{~ms}$.

For analysis methods that used cross-validation (see below), for each cue condition, we simulated network dynamics twice with independent realizations of $\eta^{(c)}(t)$, to serve as (trial-averaged) train and test data. For other analyses, we used a single set of simulated trajectories. All analyses involving networks with randomly generated (or initialized) connectivities that also did not require re-fitting their responses with other networks (Fig. 1, Fig. 2d,e, Fig. 3, Fig. 5a-d, Extended Data Fig. 1, Extended Data Fig. 3a-c, Extended Data Fig. 4, and Extended Data Figs. 6-8) were repeated a total of $n=100$ times, consisting of either 10 different networks and 10 different simulations (non-cross-validated) or simulation-pairs (cross-validated), each time with independent samples of $\eta^{(c)}(t)$ (for networks with stochastic dynamics—with one exception, see below; Fig. 1, Fig. 3, Fig. 5a-d, Extended Data Fig. 1, Extended Data Fig. 3a-c, and Extended Data Figs. 6-8), or (for deterministic networks, Fig. 2d,e and Extended Data Fig. 4a,b, as well as for the stochastic networks of Extended Data Fig. 4c,d) 100 different networks, each with a single simulation (non-cross-validated) or simulation-pair (cross-validated). For those analyses that did require the re-fitting of nonlinear networks' responses with other (linear deterministic) networks (Extended Data Fig. 3d and Fig. 5e), we used just one simulation of the original (stochastic nonlinear) network, so $n=10$ simulations in total. Analyses of linear deterministic networks (Fig. 2a-c, Fig. 4f, Fig. 5e, Extended Data Fig. 2, Extended Data Fig. 3d, and Extended Data Fig. 10f,g), used a single simulation per network as their dynamics were deterministic. Note that for (linear deterministic) networks obtained by fitting simulated neural responses, this meant that these analyses were repeated a total of $n=10$ times, once for each set of original responses that had been fit (Fig. 5e, Extended Data Fig. 3d, and Extended Data Fig. 10f,g). For (linear deterministic) networks obtained by fitting experimentally recorded neural responses, this meant that these analyses were performed either only once on the single split of the data (Fig. 4d; blue, Fig. 4e,f, and Extended Data Fig. 10g), or were repeated $n=100$ times on 100 different random time shuffles of the data (Fig. 4d; dark grey, and Extended Data Fig. 10f). 
bioRxiv preprint doi: https://doi.org/10.1101/2021.11.16.468360; this version posted December 17, 2021. The copyright holder for this preprint (which was not certified by peer review) is the author/funder, who has granted bioRxiv a license to display the preprint in perpetuity. It is made available under aCC-BY 4.0 International license.

\section{Nonlinear networks}

For nonlinear networks (Figs. 1 and 5, Extended Data Fig. 1, Extended Data Fig. 3c, and Extended Data Figs. 68), we ensured that they performed working memory maintenance competently by optimizing their free parameters, $\mathbf{W}, \mathbf{b}$, and $\mathbf{h}^{(c)}$ for appropriate cost functions (see below, Cost functions and training for nonlinear networks).

\section{Nonlinear networks with instantaneous inputs}

Following classical theoretical approaches to attractor network dynamics, we first used nonlinear neural networks in which stimulus inputs acted instantaneously to determine the initial conditions of the dynamics Fig. 1 and Extended Data Figs. 1 and 3. These networks were optimized using a 'just-in-time' cost function (Cost functions and training for nonlinear networks) under one or two constraints. First, for all these networks, we constrained stimulus inputs to have a Euclidean norm of 3 to prevent unrealistically large initial activities (relevant e.g. due to energy constraints). This also avoided the trivial solution of inputs that initialize the network directly in the attractor state (although, due e.g. to the stochastic dynamics and numerical precision, the performance of such a solution may not even be distinguishable from that of a network whose dynamics only approach the attractor state by the time the cost is applied, and so may never be found during optimization). Second, for symmetric networks, we enforced $\mathbf{W} \leftarrow \frac{1}{2}\left(\mathbf{W}+\mathbf{W}^{\top}\right)$.

These networks were trained in two epochs. For the first 1000 training iterations, we optimized all free parameters. After this, we confirmed that our trained networks did indeed have attractors (i.e. that they were attractor networks) and determined where these attractors were in state space by finding the stable fixed points of the networks' dynamics (Fig. 1e,j, and Extended Data Fig. 1b,f)—see below. We then continued for another 1000 training iterations with only optimizing the initial conditions, $\mathbf{h}^{(c)}$, while keeping the other parameters, $\mathbf{W}$ (Fig. $\left.1 \mathrm{~b}, \mathrm{~g}\right)$ and b, fixed at the values obtained at the end of the first 1000 iterations. For this, we considered three possible scenarios for introducing additional constraints on the initial conditions (beside the one on their norm, see above): they were either projected and then restricted to the persistent subspace or to the persistent nullspace (see Identifying specific subspaces in network dynamics for how these subspaces were computed), or there was no such constraint applied so that they could utilize any direction in the full state space of the network. In addition, to understand the link between the linearized (Local linearization of nonlinear dynamics) and original (above) forms of the dynamics of these networks, we also considered three more constraints on the initial conditions: constraining them to the most persistent, most amplifying, or a random subspace of the linearized dynamics (Extended Data Fig. 3a-c).

\section{Nonlinear networks with temporally extended inputs}

To more closely follow the experimental paradigms which we modelled, we also used nonlinear networks in which stimuli provided temporally extended inputs (Fig. 5 and Extended Data Figs. 6-8). To construct these networks, stimulus inputs and the weight matrix were freely optimized (Cost functions and training for nonlinear networks), without any constraints, and optimization proceeded for a full 2000 iterations, without dividing training into different epochs.

\section{Cost functions and training for nonlinear networks}

We trained networks using one of four cost functions: a 'cue-delay' cost, a 'full-delay', a 'just-in-time' cost, and an 'after-go' cost. These costs only differed in terms of the time period in which we applied the cost function. The general form of the cost function we used was a cross entropy loss plus a regularisation term:

$$
\mathcal{L}=-\alpha_{\text {nonlin }}^{(1)} \sum_{c=1}^{6}\left(\mathbf{y}^{(c)}\right)^{\top} \int_{T_{1}}^{T_{2}} \log \left(\operatorname{Softmax}\left(\mathbf{W}_{\text {out }} \mathbf{r}^{(c)}(t)+\mathbf{b}_{\text {out }}\right)\right) \mathrm{d} t+\alpha_{\text {nonlin }}^{(2)} \sum_{c=1}^{6} \int_{t_{0}}^{t_{\max }}\left\|\mathbf{r}^{(c)}(t)\right\|_{2}^{2} \mathrm{~d} t
$$

where $T_{1}$ and $T_{2}$ determine the time period in which we applied the cost, $\alpha_{\text {nonlin }}^{(1)}$ and $\alpha_{\text {nonlin }}^{(2)}$ control the relative contributions of the cross-entropy loss and firing rate regularisation, $\mathbf{W}_{\text {out }} \in \mathcal{R}^{6 \times N}$ and $\mathbf{b}_{\text {out }} \in \mathcal{R}^{6}$ include the 6 sets of 'readout' weights and biases, respectively, and $\mathbf{y}^{(c)} \in \mathcal{R}^{6}$ is a one-hot vector where $y_{i}^{(c)}= \begin{cases}1 & \text { if } i=c \\ 0 & \text { otherwise }\end{cases}$ defining the 'target' output for each cue condition. We initialized elements of the network parameters $\mathbf{W}, \mathbf{b}, \mathbf{h}^{(c)}$, as well as the readout parameters $\mathbf{W}_{\text {out }}$ and $\mathbf{b}_{\text {out }}$ from a Gaussian distribution with mean 0 and variance $1 / N$, and then optimized using gradient descent with Adam optimization ${ }^{80}$, where gradients were obtained from backpropagation through time. We used a learning rate of 0.0005 and batch sizes of 50 trials during training combined with all 6 possible cue locations (i.e. our total effective batch size was 300 ). 
bioRxiv preprint doi: https://doi.org/10.1101/2021.11.16.468360; this version posted December 17, 2021. The copyright holder for this preprint (which was not certified by peer review) is the author/funder, who has granted bioRxiv a license to display the preprint in perpetuity. It is made available under aCC-BY 4.0 International license.

\begin{tabular}{|l|l|l|l|l|l|l|}
\hline Cost function & $t_{\max }(\mathrm{s})$ & $T_{1}(\mathrm{~s})$ & $T_{2}(\mathrm{~s})$ & $\alpha_{\text {nonlin }}^{(1)}(1 / \mathrm{s})$ & $\alpha_{\text {nonlin }}^{(2)}(1)$ & Figures \\
\hline cue-delay & 2.5 & 0 & $t_{\mathrm{go}}$ & 20 & $\begin{array}{l}0.00005 \\
0.0005\end{array}$ & $\begin{array}{l}\text { Fig. } 5 \text { and Extended Data Fig. 6 } \\
\text { Extended Data Fig. 6 }\end{array}$ \\
\hline full-delay & 2.5 & 0.25 & $t_{\mathrm{go}}$ & 20 & $\begin{array}{l}0.00005 \\
0.0005\end{array}$ & $\begin{array}{l}\text { Extended Data Fig. 8 } \\
\text { Extended Data Fig. 8 }\end{array}$ \\
\hline just-in-time & 2.5 & 0.5 & $t_{\mathrm{go}}$ & 33 & $\begin{array}{l}0.00005 \\
0.00005 \\
0.0005\end{array}$ & $\begin{array}{l}\text { Fig. 1 and Extended Data Fig. 1 } \\
\text { Fig. 5 and Extended Data Fig. 6 } \\
\text { Extended Data Fig. 6 }\end{array}$ \\
\hline after-go & 2.5 & 0.7 & $t_{\mathrm{go}}$ & 33 & $\begin{array}{l}0.00005 \\
0.0005\end{array}$ & $\begin{array}{l}\text { Fig. 5 } \\
\text { Extended Data Fig. 7 }\end{array}$ \\
\hline
\end{tabular}

Table 2 | Parameters for nonlinear network optimization. All times, $t_{\max }, T_{1}$ and $T_{2}$, are relative to stimulus onset at $t=0$. Units are shown in parentheses after the name of the corresponding parameter.

See Table 2 for how we set the parameters of Eq. $3\left(T_{1}, T_{2}, t_{0}, \alpha_{\text {nonlin }}^{(1)}\right.$ and $\left.\alpha_{\text {nonlin }}^{(2)}\right)$ depending on the cost function and the level of regularization. Briefly, the cue-delay cost included both the cue (between stimulus cue onset and offset) and the delay period (between stimulus cue offset and go cue onset), the full-delay cost the included delay period but not the cue period, the just-in-time cost started between stimulus onset and the earliest go time and ended at the onset of the go cue, and the after-go cost started at go cue onset and lasted for the duration of the go cue $(0.5 \mathrm{~s})$. For simulating the random delay task (Fig. 5 and Extended Data Figs. 6-8), analogous to what animals need to solve (see below), we sampled the go time uniformly between $t_{\mathrm{go}}=0.75 \mathrm{~s}$ and $t_{\mathrm{go}}=2 \mathrm{~s}$. For just-in-time (Fig. 1) and after-go trained networks (Extended Data Fig. 7), we also used a fixed delay task with a go time of $t_{\mathrm{go}}=2 \mathrm{~s}$. For the other cost functions, networks trained on the fixed delay task yielded very similar dynamics to their counterparts trained on the variable delay task (not shown). We set $\alpha_{\text {nonlin }}^{(1)}$ and $\alpha_{\text {nonlin }}^{(2)}$ so that networks could reliably learn the task while also exhibiting relatively stable dynamics (i.e. if $\alpha_{\text {nonlin }}^{(1)} / \alpha_{\text {nonlin }}^{(2)}$ is too large, the network dynamics can explode whereas if $\alpha_{\text {nonlin }}^{(1)} / \alpha_{\text {nonlin }}^{(2)}$ is too small, the network is not able to learn the task). Nevertheless, $\alpha_{\text {nonlin }}^{(2)}$ was varied by an order of magnitude between Fig. 5 and Extended Data Figs. 6-8 to specifically test the robustness of our results to this parameter.

\section{Linear networks}

Linear networks were either constructed "de novo" (Figs. 2 and 3 and Extended Data Fig. 4), obtained by a local linearization of canonical nonlinear dynamical systems (Extended Data Fig. 2) or of nonlinear neural network dynamics (Extended Data Fig. 3a-c), or they were fitted to neural responses obtained from experiments (Fig. $4 f$ and Extended Data Fig. 10f,g) or the simulation of nonlinear networks (Fig. 5e and Extended Data Fig. 3d).

\section{De novo linear networks}

We used de novo linear networks to develop an analytical understanding of the dynamics of optimal information loading. These networks included small 2-neuron networks with hand-picked parameters chosen to illustrate the differences between normal (symmetric) and non-normal (unconstrained) dynamics and the effects of different initial conditions (Fig. 2a-c), as well as large networks (with 10,100, or 1000 neurons) with randomly generated parameters (Fig. 3 and Extended Data Fig. 4a,b). Initial conditions were determined by computing the most persistent and amplifying direction(s) based on the Jacobian of the dynamics (Figs. 2 and 3 and Extended Data Fig. 4a,b, see Identifying specific subspaces in network dynamics). Alternatively, we optimised initial conditions for maximal asymptotic overlap with the most persistent mode (Extended Data Fig. 4c,d; see below). For setting the noise level, $\sigma$, in these networks, we considered two scenarios: noise matched (Fig. 3a, light green and grey) and performance matched (Fig. 3a, dark green and black). For noise matched simulations, we first determined the highest value of $\sigma$ that still allowed us to obtain $100 \%$ decodability (using a delay-trained decoder) for all networks when receiving inputs aligned with the most amplifying mode (Fig. 3a, red). This resulted in $\sigma=0.1$ for symmetric models, and $\sigma=0.2$ for unconstrained models. We then used the same $\sigma$ for simulations using inputs aligned with the most persistent and random directions. For performance matched simulations, we used a different value of $\sigma$ for each possible input direction so that all models achieved $100 \%$ decodability using a delay-trained decoder. For symmetric models, this resulted in $\sigma=0.1$ for inputs aligned with either the persistent or most amplifying modes, and $\sigma=0.005$ for random inputs. For unconstrained models, this resulted in $\sigma=0.2$ for inputs aligned with the most amplifying mode, $\sigma=0.02$ for inputs aligned with the persistent mode, and $\sigma=0.0005$ for random inputs. (Note that, consistent with our theory, smaller noise levels were necessary to achieve the same desired level of performance for input directions that were predicted to be increasingly suboptimal by our analysis.) 
bioRxiv preprint doi: https://doi.org/10.1101/2021.11.16.468360; this version posted December 17, 2021. The copyright holder for this preprint (which was not certified by peer review) is the author/funder, who has granted bioRxiv a license to display the preprint in perpetuity. It is made available under aCC-BY 4.0 International license.

To demonstrate that the initial conditions along the most amplifying directions, obtained by control theoretic analyses, were indeed optimal for maximising the overlap with the most persistent mode (the measure of optimality we used for these networks, Fig. 2c,e), we also used a direct numerical optimisation approach, analogous to that used to optimise initial conditions in our nonlinear networks (Figs. 1 and 5, see also Cost functions and training for nonlinear networks). Specifically, we optimized $\mathbf{h}^{(c)}$ (constrained to have unit Euclidean norm) with gradient descent using Adam optimization ${ }^{80}$ with gradients obtained from back-propagation through time using the following cost function

$$
\mathcal{L}=\int_{1.5 \mathrm{~s}}^{2 \mathrm{~s}}\left[\tanh \left(\mathbf{v}_{1}^{\top} \mathbf{x}(t)\right)-1\right]^{2} \mathrm{~d} t
$$

where $\mathbf{v}_{1}$ is the eigenvector associated with eigenvalue 1 of the Jacobian (i.e. the most persistent mode). We used a learning rate of 0.0001 . We performed the above training procedure independently for 100 random networks (either symmetric or unconstrained) and we show averaged results in Extended Data Fig. 4c,d. We also used random initial conditions as controls. These had elements that were either sampled from a standard normal distribution (re-scaled to have unit Euclidean norm) in large networks (Fig. 2d,e, Fig. 3, and Extended Data Fig. 4a,b), or in the case of 2-neuron networks, quasi-randomly chosen (with unit Euclidean norm) for illustrative purposes (Fig. 2a-c).

\section{Local linearization of nonlinear dynamics}

To better understand how the dynamics of optimal information loading that we identified in linear networks apply to nonlinear attractor dynamics, we performed a local linearization of our simulated nonlinear networks (Extended Data Fig. 2 and Extended Data Fig. 3a-c). This approach required access to the "true" dynamical equations of the nonlinear networks - which we had by construction.

We performed local linearizations of the original nonlinear network dynamics in $\mathbf{x}$-space (the space of variables in which the dynamics was defined, Eq. 1) around the origin - which served as the reference point with respect to which the norm of optimized initial conditions was constrained in the networks we linearized (Nonlinear networks; analogous to our analysis of information loading in linear networks, Fig. 2a-e, and see also Subspace-constrained initial conditions). As the ReLU firing rate nonlinearity of these networks is non-differentiable at exactly the origin, we computed the "average" Jacobian of the system in the immediate vicinity of the origin instead. Because the derivative of each ReLU is 0 or 1 in half of the activity space of the network, this resulted in the Jacobian $\mathbf{J}=0.5 \mathbf{W}^{*}-\mathbf{I}$, where $\mathbf{W}^{*}$ is the weight matrix of the original nonlinear network. We then used the Jacobian we thus obtained to map the resulting linearized dynamics to a deterministic linear network with the effective weight matrix $\mathbf{W}=(\mathbf{J}+\mathbf{I})-\lambda_{\max } \mathbf{I}$, where $\lambda_{\max }$ is the largest real eigenvalue of $\mathbf{J}$. Thus, the resulting dynamics were always (marginally) stable. The bias parameters, $\mathbf{b}$, were the same as in the original nonlinear networks. The initial conditions, $\mathbf{h}^{(c)}$, were either the ones we originally optimized for the nonlinear dynamics without any constraints (beside a constraint on their norm), or they were optimized while constraining them to the most persistent, most amplifying, or a randomly chosen subspace of these linearized dynamics (all were of the same dimensionality for a fair comparison, Extended Data Fig. 3).

We used the same approach to linearize the dynamics of the canonical minimal nonlinear attractor dynamics that we used to gain insights into information loading in nonlinear systems (Canonical nonlinear systems with two stable fixed points, see also Supplementary Math Note and Extended Data Fig. 2). In this case, the Jacobian was well defined at the origin, so there was no need to average it. For consistency with the notation and terminology we use in the rest of this paper, and without loss of generality (as linear dynamical systems and linear neural networks are isomorphic), we refer to the resulting linear dynamical system as a "linear neural network" and define it by its "effective" weight matrix (defined via the Jacobian as above). Initial conditions were magnitudematched and chosen to align with the most persistent or the most amplifying direction extracted from the Jacobian (Identifying specific subspaces in network dynamics), or chosen randomly, or varied systematically to cover the whole range of possible directions. There were no other parameters for these linearized "networks".

\section{Fitting linear neural networks to neural responses}

In order to be able to apply our theoretically derived measures of optimal information loading without having access to the true dynamics of the system, we also created linear neural networks whose parameters were fitted to experimental data (see below). As a control, we repeated the same fitting procedure with simulated nonlinear networks to validate that our approach provides meaningful results when 1 . we do not have access to the true dynamics but only to samples of activities generated by those dynamics, and 2 . we also cannot assume that the true dynamics are linear. 
bioRxiv preprint doi: https://doi.org/10.1101/2021.11.16.468360; this version posted December 17, 2021. The copyright holder for this preprint (which was not certified by peer review) is the author/funder, who has granted bioRxiv a license to display the preprint in perpetuity. It is made available under aCC-BY 4.0 International license.

We fitted deterministic linear neural networks to $1 \mathrm{~s}$ of trial-averaged neural activity (experimentally recorded, or simulated by a nonlinear neural network model). For the main analyses (Fig. 4d-f, Fig. 5e, Extended Data Fig. 3d, and Extended Data Fig. 10f), we used data starting from the onset of the stimulus cue. For the control analysis of late delay experimental recordings (Extended Data Fig. 10g), we used the final $1 \mathrm{~s}$ of neural activity just prior to the go cue. For the shuffle control (Fig. 4d; dark grey, and Extended Data Fig. 10f), we again used data starting from stimulus onset but randomly shuffled neural activity across time and proceeded by fitting this shuffled data instead.

For fitting high dimensional neural data, we first performed principal components analysis on neural activity (dimensions: neurons, data points: time points, indexed by $t$, and cue conditions, indexed by $c$ ), and projected it through the principal components (PCs): $\mathbf{x}_{*}^{(c)}(t)=\mathbf{P} \mathbf{r}_{*}^{(c)}(t)$, where the columns of $\mathbf{P}$ are top 20 principal components of the data, and $\mathbf{r}_{*}^{(c)}(t)$ is trial averaged neural responses (mean-centered, see above) at time $t$ in condition c. These top 20 PCs captured approximately $75 \%$ and $76 \%$ of variance for monkeys $\mathrm{K}$ and T, respectively during the cue and early delay period (Fig. 4f), $70 \%$ and $60 \%$ of variance for monkeys $\mathrm{K}$ and $\mathrm{T}$, respectively during the late delay period (Extended Data Fig. $10 \mathrm{~g}$ ), and over $95 \%$ of the variance for all simulated neural activities (Fig. 5e). The projected neural activity time courses of the neural data, $\mathbf{x}_{*}^{(c)}(t)$, served as the targets that needed to be matched (after a suitable linear transformation with "read-out" matrix $\mathbf{C} \in \mathcal{R}^{20 \times 20}$ ) by the neural activity time courses generated by the fitted neural network's dynamics in the corresponding cue conditions, $\mathbf{x}^{(c)}(t)$ (Eqs. 1 and 2). For fitting the parameters of the network $\left(\mathbf{W}, \mathbf{h}^{(c)}, \mathbf{b}\right)$ and the readout matrix $(\mathbf{C})$, we used the following cost function:

$$
\begin{aligned}
\mathcal{L} & =\varepsilon^{2}+\alpha_{\operatorname{lin}}\left[\|\mathbf{C}\|_{\mathrm{F}}^{2}+\|\mathbf{b}\|_{2}^{2}+\sum_{c=1}^{6}\left\|\mathbf{h}^{(c)}\right\|_{2}^{2}\right] \\
\text { with } \quad & \varepsilon^{2}=\frac{1}{6} \sum_{c=1}^{6} \int_{0 \mathrm{~s}}^{1 \mathrm{~s}}\left(\mathbf{e}^{(c)}(t)\right)^{\top} \mathbf{D} \mathbf{e}^{(c)}(t) \mathrm{d} t \\
\text { and } \quad \mathbf{e}^{(c)}(t) & =\mathbf{C} \mathbf{x}^{(c)}(t)-\mathbf{x}_{*}^{(c)}(t)
\end{aligned}
$$

being the mean squared error of the fit

the momentary error

where $\mathbf{D}$ is a diagonal matrix with the variances explained by the corresponding PCs in $\mathbf{P}$ on the diagonal (encouraging the optimization procedure to prioritize fitting the top PCs), $\|\cdot\|_{\mathrm{F}}^{2}$ is the Frobenius norm of a matrix.

Although including $\mathbf{C}$ as an additional parameter (free to be optimized) makes the fitting problem overparametrized (at least with respect to the fitting error, $\varepsilon^{2}$, as for any invertible choice of $\mathbf{G}$, any remapping of the parameters as $\mathbf{W} \rightarrow \mathbf{G ~ W ~ G} \mathbf{G}^{-1}, \mathbf{h}^{(c)} \rightarrow \mathbf{G} \mathbf{h}^{(c)}, \mathbf{b} \rightarrow \mathbf{G} \mathbf{b}$, and $\mathbf{C} \rightarrow \mathbf{C ~ G}^{-1}$ achieves the same $\varepsilon^{2}$ ), we included $\mathbf{C}$ because it allowed the network to develop dynamics that make appropriate use of persistent and amplifying modes without simultaneously having to match the neural dimensions (which can be easily re-mapped with $\mathbf{C}$ ). We then used $\mathbf{C}$ as the read-out matrix when identifying amplifying modes (see Identifying specific subspaces in network dynamics). Indeed, when validating this fitting procedure with simulated responses generated by linear stochastic neural networks with different (instantaneous) information loading strategies (most persistent, most amplifying, random; see Subspace-constrained initial conditions), and using our standard subspace-overlap-based measures (Measures of subspace overlap) to identify the information loading strategy from these simulated responses, we found that the true information loading strategy was recovered more reliably with including $\mathbf{C}$ than without it (not shown). Moreover, we found this approach was even able to distinguish between different information loading strategies of nonlinear networks from simulated data (Extended Data Fig. 3d).

Also note that we had no constraints on $\mathbf{W}$ to define stable dynamics. Nevertheless, when fitting experimental recordings, and responses generated by nonlinear attractor networks, we found that the largest real eigenvalue of the fitted $\mathbf{W}$ was typically within the $0.95 \leq \lambda_{\max } \leq 1.05$ range, i.e. the dynamics were near marginal stability, in line with the dynamics of our de novo linear neural networks (De novo linear networks), as well as of those that we obtained by local linearization (Local linearization of nonlinear dynamics). The only exception was when fitting the responses of nonlinear networks trained on an after-go-time cost (Cost functions and training for nonlinear networks) which resulted in dynamics without attractors and, consequently, the fitted linear dynamics had $\lambda_{\max }>$ 1.05 .

We used Adam ${ }^{80}$ to perform gradient descent optimization of $\mathbf{W}, \mathbf{h}^{(c)}$, $\mathbf{b}$, and $\mathbf{C}$ with gradients obtained from back-propagation through time, and a learning rate of 0.0001 . We initialized elements of all of these parameters from a Gaussian distribution with mean 0 and variance $1 / 20$. We set the regularisation parameter to $\alpha_{\text {lin }}=1 / 12$, although we found that the results did not change substantially when setting $\alpha_{\text {lin }}=0$ or using larger values of $\alpha_{\text {lin }}$.

In most cases (Fig. 4f, Fig. 5e, and Extended Data Fig. 10f), we set $\mathbf{x}^{(c)}\left(t_{0}\right)=\mathbf{0}$. There were two exceptions to this. First, when fitting the late delay dynamics in the experimental recordings (Extended Data Fig. 10g), we set $\mathbf{x}^{(c)}\left(t_{0}\right)=\mathbf{C}^{-1} \mathbf{x}_{*}^{(c)}\left(t_{0}\right)$ (i.e. we fixed the initial condition of the latent dynamics to the data; we also observed qualitatively similar results when we included $\mathbf{x}^{(c)}\left(t_{0}\right)$ as a separate optimizable parameter in this case). Second, 
bioRxiv preprint doi: https://doi.org/10.1101/2021.11.16.468360; this version posted December 17, 2021. The copyright holder for this preprint (which was not certified by peer review) is the author/funder, who has granted bioRxiv a license to display the preprint in perpetuity. It is made available under aCC-BY 4.0 International license.

when fitting simulated data from models that used instantaneous stimulus inputs (Extended Data Fig. 3d), we set $\mathbf{x}^{(c)}\left(t_{0}\right)=\mathbf{h}^{(c)}$.

\section{Previous working memory models}

We used the following dynamics for implementing all previous neural network models of working memory:

$$
\begin{gathered}
\mathbf{x}^{(c)}=\mathbf{W} \mathbf{r}^{(c)}(t)+m_{\mathrm{h}}(t) \mathbf{h}^{(c)}+m_{\mathrm{g}}(t) \mathbf{g} \\
\tau_{\mathrm{r}} \frac{\mathrm{d} \mathbf{r}^{(c)}(t)}{\mathrm{d} t}=-\mathbf{r}^{(c)}(t)+\mathbf{f}\left(\mathbf{x}^{(c)}(t)\right)+\mathbf{b}_{\mathrm{r}}+\sigma_{\mathrm{r}} \boldsymbol{\eta}_{\mathrm{r}}^{(c)}(t)
\end{gathered}
$$

where all symbols refer to the same (or a closely analogous, see below) quantity as in Eqs. 1 and 2. Note that we use this notation to best expose the similarities with and differences from the dynamics of our networks (Eqs. 1 and 2), rather than the original notation used for describing these models $7,11,33$, but the dynamics are nevertheless identical to those previously published. Overall, these dynamics are closely analogous to those that we used earlier for our networks with the following differences. First, for us, dynamics were defined in $\mathbf{x}$-space, with $\mathbf{r}$ being an instantaneous function of $\mathbf{x}$. Here, the dynamics are defined instead in $\mathbf{r}$-space (Extended Data Fig. 5), with $\mathbf{x}$ being an instantaneous function of $\mathbf{r}$. (There are slightly different assumptions underlying these ratebased formulations of neural network dynamics when deriving them as approximations of the dynamics of spiking neural networks ${ }^{48}$, and the two become identical in the case of linear dynamics.) As a result, time constants, $\tau_{\mathrm{r}}$, biases, $\mathbf{b}_{\mathrm{r}}$, and the variance of noise, $\boldsymbol{\sigma}_{\mathrm{r}}$ (as well as noise itself, $\boldsymbol{\eta}_{\mathrm{r}}^{(c)}(t)$ ), are defined for $\mathbf{r}$ rather than $\mathbf{x}$. For nonlinear variants of these networks, there are also differences for the choice of single neuron nonlinearities, $\mathbf{f}(\cdot)$. Furthermore, some of these networks distinguish between excitatory and inhibitory cells, with different time constants, and noise standard deviations. Thus, each of these parameters is represented as a diagonal matrix, $\tau_{\mathrm{r}}$ and $\sigma_{\mathrm{r}}$, respectively, with each element on the diagonal storing one of two possible values of that parameter depending on the type (excitatory or inhibitory) of the corresponding neuron $\left(\tau_{\mathrm{r}}^{\mathrm{E}}\right.$ and $\sigma_{\mathrm{r}}^{\mathrm{E}}$, or $\tau_{\mathrm{r}}^{\mathrm{I}}$ and $\sigma_{\mathrm{r}}^{\mathrm{I}}$, respectively). Most importantly, all of these networks used a set of parameters which were hand-crafted to produce the required type of dynamics, rather than optimized for a function (or to fit data) as in the case of our networks. In line with our analyses of experimental data and function-optimized networks (Figs. 4 and 5), simulations started at $t_{0}=-0.5 \mathrm{~s}$, i.e. $0.5 \mathrm{~s}$ before stimulus cue onset (defined as $t=0$ ), the stimulus cue lasted for $0.25 \mathrm{~s}$, and the go cue appeared at $t_{\mathrm{go}}=2 \mathrm{~s}$ and lasted for $0.5 \mathrm{~s}$. (Note that for these networks we considered the fixed-delay variant of the task as that is what these networks were originally constructed to solve.) As with our networks (Neural network models: overview), we solved the dynamics of Eqs. 8 and 9 using a first-order Euler-Maruyama approximation between $t_{0}$ and the simulation end time with a discretization time step of $1 \mathrm{~ms}$.

For analysis methods that used cross-validation (see below), we simulated network dynamics twice (for each cue condition) with independent realizations of $\eta_{r}^{(c)}(t)$, to serve as (trial-averaged) train and test data. For other analyses, we used a single set of simulated trajectories. All analyses involving these networks were repeated $n=10$ times, using 10 different simulations (non-cross-validated) or simulation-pairs (cross-validated), each time with independent samples of $\eta_{\mathrm{r}}^{(c)}(t)$.

Table 3 provides the values of most network and other parameters used for simulating each model. In the following we provide the additional details for each of these models that are not included in Table 3.

\section{Discrete attractor model}

The discrete attractor model that we used (Extended Data Fig. 5a) has been described previously (see the methods of Ref. 7). The model contained separate excitatory and inhibitory populations.

The weight matrix was of the form

$$
\mathbf{W}=\left(\begin{array}{ll}
\mathbf{W}^{\mathrm{EE}} & -\mathbf{W}^{\mathrm{IE}} \\
\mathbf{W}^{\mathrm{EI}} & -\mathbf{W}^{\mathrm{II}}
\end{array}\right)
$$

where the elements of $\mathbf{W}^{\mathrm{IE}}, \mathbf{W}^{\mathrm{EI}}$, and $\mathbf{W}^{\mathrm{II}}$ were set to $2.4 / \mathrm{N}, 8 / \mathrm{N}$, and $2.6 / \mathrm{N}$, respectively. The excitatory sub-matrix $\mathrm{W}^{\mathrm{EE}}$ was constructed by dividing the population of excitatory cells into six clusters (of 9 neurons each), with each cluster corresponding to one of the stimulus cue conditions. Connections within each cluster were strong, with a value of $30 / \mathrm{N}$. Connections between neurons belonging to clusters that corresponded to adjacent stimulus cues were weaker, with a value of $2.5 / \mathrm{N}$. All other connections were very weak, with a value of $0.02 / \mathrm{N}$. This resulted in a block circulant structure for $\mathrm{W}^{\mathrm{EE}}$.

Stimulus cue inputs were set to

$$
h_{i}^{(c)} \propto \frac{350 e^{8 \cos \left(\pi\left(\frac{4 i}{N}-\frac{2 c-1}{6}\right)\right)}}{\sum_{k=1}^{N / 2} e^{8 \cos \left(\pi\left(\frac{4 k}{N}-\frac{2 c-1}{6}\right)\right)}}
$$




\begin{tabular}{|c|c|c|c|c|c|c|}
\hline \multicolumn{7}{|c|}{ Parameters used in network simulations of previous models } \\
\hline Symbol & $\begin{array}{l}\text { Extended Data } \\
\text { Fig. } 5 \mathrm{a}\end{array}$ & $\begin{array}{l}\text { Extended Data } \\
\text { Fig. 5b }\end{array}$ & $\begin{array}{l}\text { Extended Data } \\
\text { Fig. } 5 \mathrm{c}\end{array}$ & $\begin{array}{l}\text { Extended Data } \\
\text { Fig. } 5 \mathrm{~d}\end{array}$ & Units & Description \\
\hline $\mathrm{N}$ & 108 & 100 & 100 & 100 & - & number of neurons \\
\hline$\tau$ & - & - & 0.01 & 0.05 & $\mathrm{~s}$ & membrane time constant \\
\hline$\tau_{\mathrm{r}}^{\mathrm{E}}$ & 0.02 & 0.02 & - & - & $\mathrm{s}$ & $\begin{array}{l}\text { membrane time constant } \\
\text { (E neurons) }\end{array}$ \\
\hline$\tau_{\mathrm{r}}^{\mathrm{I}}$ & 0.01 & 0.01 & - & - & $\mathrm{s}$ & $\begin{array}{l}\text { membrane time constant } \\
\text { (I neurons) }\end{array}$ \\
\hline$t_{0}$ & -0.5 & -0.5 & -0.5 & -0.5 & $\mathrm{~s}$ & simulation start time \\
\hline $\mathbf{r}^{(c)}\left(t_{0}\right)$ & $\mathbf{0}$ & $\mathbf{0}$ & $\mathbf{0}$ & $\mathbf{0}$ & $\mathrm{Hz}$ & initial condition \\
\hline $\mathbf{f}(\cdot)$ & nonlinear $^{a}$ & nonlinear $^{a}$ & $\operatorname{linear}^{a}$ & linear ${ }^{a}$ & $\mathrm{~Hz}$ & neural activation function \\
\hline W & $\operatorname{set}^{b}$ & $\operatorname{set}^{b}$ & $\operatorname{set}^{b}$ & $\operatorname{set}^{b}$ & $\mathrm{~s}$ & weight matrix \\
\hline C & 6 & 6 & 6 & 6 & - & number of stimuli \\
\hline $\mathbf{h}^{(c)}$ & $\operatorname{set}^{b}$ & $\operatorname{set}^{b}$ & $\operatorname{set}^{b}$ & $\operatorname{set}^{b}$ & - & stimulus input \\
\hline g & $\sum_{c} \mathbf{h}^{(c)}$ & $\sum_{c} \mathbf{h}^{(c)}$ & $\sum_{c} \mathbf{h}^{(c)}$ & $\sum_{c} \mathbf{h}^{(c)}$ & - & go cue \\
\hline$m_{\mathrm{h}}(t)$ & $K(0,0.25)^{c}$ & $K(0,0.25)^{c}$ & $K(0,0.25)^{c}$ & $K(0,0.25)^{c}$ & - & stimulus masking kernel \\
\hline$m_{\mathrm{g}}(t)$ & $K\left(t_{\mathrm{go}}, t_{\mathrm{go}}+0.5\right)^{c}$ & $K\left(t_{\mathrm{go}}, t_{\mathrm{go}}+0.5\right)^{c}$ & $K\left(t_{\mathrm{go}}, t_{\mathrm{go}}+0.5\right)^{c}$ & $K\left(t_{\mathrm{go}}, t_{\mathrm{go}}+0.5\right)^{c}$ & - & go cue masking kernel \\
\hline $\mathbf{b}_{\mathrm{r}}$ & $\begin{array}{l}b_{\mathrm{r}}^{\mathrm{E}}=-1.2 \\
b_{\mathrm{r}}^{\mathrm{I}}=0.28\end{array}$ & $\begin{array}{l}b_{\mathrm{r}}^{\mathrm{E}}=0.2, \\
b_{\mathrm{r}}^{\mathrm{I}}=0.5\end{array}$ & $\mathbf{0}$ & $\mathbf{0}$ & $\mathrm{Hz}$ & cue-independent bias \\
\hline$\sigma_{\mathrm{r}}$ & - & - & 0.02 & 0.02 & $\mathrm{~Hz}$ & noise standard deviation \\
\hline$\sigma_{\mathrm{r}}^{\mathrm{E}}$ & 2 & 1 & - & - & $\mathrm{Hz}$ & $\begin{array}{l}\text { noise standard deviation } \\
\text { (E neurons) }\end{array}$ \\
\hline$\sigma_{\mathrm{r}}^{\mathrm{I}}$ & 1 & 3 & - & - & $\mathrm{Hz}$ & $\begin{array}{l}\text { noise standard deviation } \\
\text { (I neurons) }\end{array}$ \\
\hline
\end{tabular}

Table 3 | Parameters used in previous models.

a For nonlinear networks, $f_{i}(\mathbf{x})=\left\{\begin{array}{ll}{\left[x_{i}\right]_{+}^{2}} & \text { if } x_{i} \leq 1, \\ \sqrt{4 x_{i}-3} & \text { otherwise. }\end{array}\right.$. For linear networks $f_{i}(\mathbf{x})=x_{i}$.

${ }^{b}$ See text for details.

${ }^{c} K\left(t_{1}, t_{2}\right)= \begin{cases}1 & \text { if } t_{1} \leq t \leq t_{2} \mathrm{~s}, \\ 0 & \text { otherwise. }\end{cases}$ 
for cues $c=1, \ldots, 6$ and cells $i=1, \ldots, N / 2$ (i.e. inputs were only delivered to the excitatory neurons).

\section{Bump attractor model}

The bump attractor model that we used (Extended Data Fig. 5b) has been described previously (see Ref. 7). The model contained separate excitatory and inhibitory populations. As in the discrete attractor model, the weight matrix was of the form

$$
\mathbf{W}=\left(\begin{array}{ll}
\mathbf{W}^{\mathrm{EE}} & -\mathbf{W}^{\mathrm{IE}} \\
\mathbf{W}^{\mathrm{EI}} & -\mathbf{W}^{\mathrm{II}}
\end{array}\right)
$$

where the elements of $\mathbf{W}^{\mathrm{IE}}, \mathbf{W}^{\mathrm{EI}}$, and $\mathbf{W}^{\mathrm{II}}$ were set to $6.8 / \mathrm{N}, 8 / \mathrm{N}$, and $1.7 / \mathrm{N}$, respectively. The excitatory sub-matrix $\mathrm{W}^{\mathrm{EE}}$ had a circulant form:

$$
W_{i j}^{\mathrm{EE}}=\frac{6 e^{1.5 \cos \left(\frac{4 \pi(i-j)}{N}\right)}}{\sum_{k=0}^{N / 2-1} e^{1.5 \cos \left(\frac{4 \pi k}{N}\right)}}
$$

for cell-pairs $i, j=1, \ldots, N / 2$.

Stimulus cue inputs were also analogous to those used in the discrete attractor models and were set to

$$
h_{i}^{(c)}=\frac{200 e^{1.5 \cos \left(\pi\left(\frac{4 i}{N}-\frac{2 c-1}{6}\right)\right)}}{\sum_{k=1}^{N / 2} e^{1.5 \cos \left(\pi\left(\frac{4 k}{N}-\frac{2 c-1}{6}\right)\right)}}
$$

for cues $c=1, \ldots, 6$ and cells $i=1, \ldots, N / 2$ (i.e., as above, inputs were only delivered to the excitatory neurons).

\section{Feedforward network model}

The linear feedforward network model that we used (Extended Data Fig. 5c) has been described previously (see Refs. 18,33). (For pedagogical purposes, we used the simplest set up consisting of a feedforward chain of neurons, see below. However, using a more general network model that contained 'hidden' feedforward chains ${ }^{18}$ did not affect our analyses except for Extended Data Fig. 10e which, in contrast to the simple feedforward chain, could display overlap values greater than 0.5 .) There were no separate excitatory and inhibitory populations in this model, and the weight matrix included a single chain running from neuron 1 to neuron $N$ :

$$
W_{i j}=\delta_{(i-1), j}
$$

for cell-pairs $i, j=1, \ldots, N$.

The stimulus cues provided random inputs delivered to only the first 10 neurons so that each input could pass through the feedforward network:

$$
h_{i}^{(c)} \stackrel{\text { iid. }}{\sim} \mathcal{N}(0,1)
$$

for cues $c=1, \ldots, 6$ and cells $i=1, \ldots, 10$.

\section{Linear integrator model}

The linear integrator model that we used (Extended Data Fig. 5d) has been described previously (see Ref. 11). There were no separate excitatory and inhibitory populations in this model, and the weight matrix was constructed such that network dynamics were non-normal, non-oscillatory, and stable with a single two-dimensional neutrally stable subspace (i.e. a plane attractor). We achieved this by defining $\mathrm{W}$ via its eigen-decomposition:

$$
\mathbf{W}=\mathbf{V} \mathbf{D V}^{-1}
$$

where the eigenvectors (columns of $\mathbf{V}$, denoted as $\mathbf{v}_{j}$, for $j=1, \ldots, N$, with elements $v_{i j}$, for $i, j=1, \ldots, N$ ) were generated by the following process:

1. Generating a random vector:

$$
\nu_{i} \stackrel{\text { iid. }}{\sim} \mathcal{N}(0,1)
$$

for $i=1, \ldots, N$. 
2. Making the first $10 \%$ of vectors overlapping so that the resulting matrix is non-normal:

$$
\begin{gathered}
v_{i k}=\nu_{i}+\epsilon_{i k} \\
\text { where } \epsilon_{i k} \stackrel{\text { iid. }}{\sim} \mathcal{N}\left(0,0.05^{2}\right)
\end{gathered}
$$

for $i=1, \ldots, N$ and $k=1, \ldots, K$ with $K=0.1 \mathrm{~N}$.

3. Making the the other $90 \%$ of vectors orthogonal:

$$
\mathbf{v}_{K+k}=\text { the } k \text { th column of } \operatorname{Nullspace}\left(\mathbf{v}_{1}, \ldots \mathbf{v}_{K}\right)
$$

for $k=1, \ldots, N-K$

4. Unit normalizing each vector:

$$
\mathbf{v}_{k} \leftarrow \frac{\mathbf{v}_{k}}{\left\|\mathbf{v}_{k}\right\|_{2}^{2}}
$$

and the eigenvalues $\left(\lambda_{i}\right.$, for $i=1, \ldots, N$, the diagonal elements of the diagonal matrix $\mathbf{D}$ ) were generated by the following process

1. Generating random (real) values:

$$
\lambda_{i} \stackrel{\text { iid. }}{\sim} \text { Uniform }(0,0.8)
$$

for $i=1, \ldots, N-2$.

2. Creating a pair of neutrally stable eigenmodes:

$$
\lambda_{N-1}=\lambda_{N}=1
$$

The stimulus cue inputs were set to

$$
\mathbf{h}^{(c)}=\mathbf{K}\left(\begin{array}{c}
\cos \left(\frac{(c-1) \pi}{3}\right) \\
\sin \left(\frac{(c-1) \pi}{3}\right) \\
1
\end{array}\right)
$$

for cues $c=1, \ldots, 6$, and we considered two forms for $\mathbf{K}$ : either $\mathbf{K}=\left[\mathbf{v}_{N-1}, \mathbf{v}_{N}, \mathbf{v}_{r_{1}}\right]+\left[\mathbf{v}_{r_{2}}, \mathbf{v}_{r_{3}}, \mathbf{v}_{r_{4}}\right]$ (Extended Data Fig. $5 \mathrm{~d}$, left; as in the original formulation ${ }^{11}$ ) or $\mathbf{K}=\left[\mathbf{v}_{r_{1}}, \mathbf{v}_{r_{2}}, \mathbf{v}_{r_{3}}\right]$ (Extended Data Fig. $5 \mathrm{~d}$, right), where $r_{1}, r_{2}, r_{3}, r_{4}$ were randomly drawn integers over the range 1 to $N-2$. The first formulation of $K$ ensured that stimulus cue inputs partially align with the persistent subspace, whereas the second formulation of $\mathbf{K}$ ensured that stimulus cue inputs align only with random directions.

\section{Canonical nonlinear systems with two stable fixed points}

In order to illustrate the applicability of our analysis of optimal information loading in linear dynamical systems to the behaviour of nonlinear dynamical systems, we first studied two variants (either symmetric or non-symmetric) of a canonical nonlinear system that can exhibit two stable fixed points. (These systems are closely related to the damped, unforced Duffing oscillator which is a classic example of a [non-symmetric] system that can exhibit two stable fixed points. Additionally, the analysis of these systems also holds for the Duffing oscillator.)

The dynamics of the first system (which has a symmetric Jacobian matrix) are governed by

$$
\begin{aligned}
& \frac{\mathrm{d} x_{1}(t)}{\mathrm{d} t}=x_{1}(t)-x_{1}^{3}(t) \\
& \frac{\mathrm{d} x_{2}(t)}{\mathrm{d} t}=-x_{2}(t)
\end{aligned}
$$

and the dynamics of the second system (which has a non-symmetric Jacobian matrix) are governed by:

$$
\begin{aligned}
& \frac{\mathrm{d} x_{1}(t)}{\mathrm{d} t}=x_{1}(t)-x_{1}^{3}(t)+3 x_{2}(t) \\
& \frac{\mathrm{d} x_{2}(t)}{\mathrm{d} t}=-x_{2}(t)
\end{aligned}
$$

We used a cubic polynomial in Eqs. 26 and 27 because it is the lowest order polynomial that allows a system to exhibit 2 stable fixed points. Both systems exhibit 3 fixed points: both have a saddle point at the origin and both have 2 asymptotically stable fixed points at $( \pm 1,0)$ (see Extended Data Fig. 2 for the state space dynamics of these two systems).

We solved the dynamics of Eqs. 26 and 27 using a first-order Euler approximation starting from $t=0$ with a discretization time step of 0.02 (note time was unitless for this model). 
bioRxiv preprint doi: https://doi.org/10.1101/2021.11.16.468360; this version posted December 17, 2021. The copyright holder for this preprint (which was not certified by peer review) is the author/funder, who has granted bioRxiv a license to display the preprint in perpetuity. It is made available under aCC-BY 4.0 International license.

\section{Analysis methods}

Here we describe methods that we used to analyse neural data. Whenever applicable, the same processing and analysis steps were applied to both experimentally recorded and model simulated data. As a first step in all our analyses, in line with previous work analysing neural population dynamics ${ }^{73}$, we removed the stimulus cueindependent time-varying mean activity from each neuron's firing rate time series (see Fig. 4a for an example). (This was done separately for training and test data for cross-validated analyses, see below.) In most of our analyses, neural activities were aligned to stimulus cue onset defined to be at $t=0$. However, due to the variable delay duration of the task (Fig. 1a), experimentally recorded neural activities were also aligned to go cue onset for analyses that required incorporating the late delay and go epochs (i.e. beyond the first $1.65 \mathrm{~s}$ after the stimulus cue onset; Fig. 4b-c, Extended Data Fig. 10a-c,g). For simulated neural activities, this was not necessary, as we always simulated our networks in a fixed-delay task for ease of analysis, even if they were optimized for a variable-delay task in accordance with how our experimental monkey subjects were trained.

\section{Identifying specific subspaces in network dynamics}

In order to understand the dynamics of neural networks with potentially complex and high-dimensional dynamics, and the way these dynamics depend on initial conditions, we identified specific subspaces within the full state space of these networks that were of particular relevance for our analyses. These subspaces served dual roles. First, as 'intervention tools', to ascertain their causal roles in high dimensional network dynamics, we used them to constrain the initial conditions of the dynamics of our networks (see also Subspace-constrained initial conditions). Second, as 'measurement tools', to reveal key aspects of the high-dimensional dynamics of neural networks, we used them to project high-dimensional neural trajectories into these lower dimensional subspaces (see also Measures of subspace overlap).

Our main analyses relied on identifying the most persistent and most amplifying modes of a network. This required dynamics that were linear-either by construction, or by (locally) linearizing or linearly fitting dynamics that were originally nonlinear (see Table 1). We computed the most persistent mode(s) in one of two different ways. First, for networks that were guaranteed to have stable dynamics by construction (i.e. those constructed de novo or by local linearization; Figs. 2 and 3 and Extended Data Fig. 2, Extended Data Fig. 3a-c, Extended Data Fig. 4, and Extended Data Fig. 5d,f), we simply used the eigenvector(s) of the weight matrix W associated with the eigenvalue(s) that had the largest real part(s). Second, for networks that were fitted to nonlinear dynamics or recorded data, and whose dynamics could thus not be guaranteed to be stable (Fig. 4f, Fig. 5e, Extended Data Fig. 3d, and Extended Data Fig. 10f,g), we used the eigenvectors of $\mathbf{W}$ associated with the largest real eigenvalues that were less than or equal to $1+\delta$ (with $\delta=0.05$ ) (i.e. we find the slowest, or most persistent, modes of the network - the $\delta$ was mostly relevant only for the after-go-time networks of Fig. 5 and Extended Data Fig. 7 which exhibited eigenvalues substantially greater than 1 and setting $\delta$ less than 0.05 did not substantially change our results).

For computing the most amplifying modes, we performed an eigendecomposition of the associated Observability Gramian $\mathbf{Q}^{53,56}$. Specifically, we obtained $\mathbf{Q}$ by solving the following Lyapunov equation:

$$
(\tilde{\mathbf{W}}-\mathbf{I})^{\top} \mathbf{Q}+\mathbf{Q}(\tilde{\mathbf{W}}-\mathbf{I})+\mathbf{C}^{\top} \mathbf{C}=\mathbf{0}
$$

where $\tilde{\mathbf{W}}$ is the "stabilized" weight matrix of the dynamics (and the $-\mathbf{I}$ terms represent the effect of the leak on the Jacobian of the dynamics, Eq. 1) and $\mathbf{C}$ is the read-out matrix of the network. The most amplifying mode(s) of the network are given as the eigenvector(s) of $\mathbf{Q}$ associated with the largest eigenvalue(s). Again, for networks that were guaranteed to have stable dynamics by construction (Figs. 2 and 3, Extended Data Fig. 3ac, Extended Data Fig. 4, and Extended Data Fig. 5 d,f), $\tilde{\mathbf{W}}=\mathbf{W}-\epsilon \mathbf{I}$, where $\mathbf{W}$ is the original weight matrix of the dynamics and $\epsilon=0.01$. For other networks, i.e. either linear networks fitted to experimental data (Fig. $4 f$ and Extended Data Fig. 10f,g), linear networks fitted to simulated nonlinear dynamics (Fig. 5e and Extended Data Fig. 3d), or local linearizations of nonlinear dynamics (Extended Data Fig. 2 and Extended Data Fig. 3ac), we used $\tilde{\mathbf{W}}=\mathbf{W}-\left(\lambda_{\max }-1+\epsilon\right) \mathbf{I}$, where $\lambda_{\max }$ is the largest real eigenvalue of $\mathbf{W}$, to ensure that the linear dynamics with $\tilde{\mathbf{W}}$ were stable (which is essential for calculating $\mathbf{Q}$ ). For networks obtained by fitting neural responses (experimentally recorded or simulated; Fig. 4f, Fig. 5e, Extended Data Fig. 3d, and Extended Data Fig. 10f,g), $\mathbf{C}$ was obtained by fitting those responses (Fitting linear neural networks to neural responses), as we wanted to understand how the fitted dynamics taking place in a latent space can generate the most discriminable fluctuations in (the principal components of) the neural responses to which they are related by this read-out matrix (although using $\mathbf{C}=\mathbf{I}$ did not change our results substantially). For all other networks (Figs. 2 and 3 , Extended Data Fig. 3a-c, Extended Data Fig. 4, and Extended Data Fig. 5d,f), we simply used $\mathbf{C}=\mathbf{I}$, as the activity of these networks was supposed to be read out in the same space within which their dynamics took place.

We also applied methods which did not rely on the linearization (or linear fitting) of network dynamics. Our goal was to develop basic intuitions for how much the dynamics of the different simulated nonlinear networks of Fig. 1 
bioRxiv preprint doi: https://doi.org/10.1101/2021.11.16.468360; this version posted December 17, 2021. The copyright holder for this preprint (which was not certified by peer review) is the author/funder, who has granted bioRxiv a license to display the preprint in perpetuity. It is made available under aCC-BY 4.0 International license.

and Extended Data Fig. 1 used the persistent subspace of their dynamics. For this, we determined the 'persistent subspace' as the subspace spanned by the 5 principal components of the final $500 \mathrm{~ms}$ of neural activities (x) across all 6 cue conditions, corresponding to 6 distinct attractors, and the 'persistent nullspace' of the network as the 45-dimensional subspace orthogonal to the persistent subspace. For plots showing the projection of network activities within the persistent subspace (Extended Data Fig. 1b,f and Extended Data Fig. 1c-d and g-h, bottom) we used the first two principal components of the full, five-dimensional persistent subspace of the network, as determined above. For plots showing the projection of network activities to persistent vs. cue-aligned directions (Fig. 1e,j, and Extended Data Fig. 1c-d and g-h, top right), 'persistent PC1' was determined as the direction spanning the two persistent states corresponding to the two cue conditions being illustrated (i.e. as above, spanning the final $500 \mathrm{~ms}$ of neural activities across the two cue conditions), and 'initial PC1 (orthogonalized)' was determined as the the direction spanning the two initial conditions corresponding to the two cue conditions being illustrated, orthogonalized with respect to the corresponding persistent PC1.

\section{Subspace-constrained initial conditions}

When using the subspaces identified above as 'intervention tools', to constrain the initial conditions of our networks, we either used the single top most persistent or amplifying mode for linear networks with low-dimensional coding spaces (including the linearized canonical nonlinear attractor dynamical system; Figs. 2 and 3 and Extended Data Figs. 2 and 4), or numerically optimized initial conditions within the corresponding higher-dimensional subspaces (Fig. 1f,k, Extended Data Fig. 1c,d,g,h, Extended Data Fig. 3; see also Nonlinear networks and Linear networks). When the persistent subspace was extracted from neural responses (rather than from the dynamical equations of the network, Identifying specific subspaces in network dynamics; Fig. 1f,k, Extended Data Fig. 1c,d,g,h, Extended Data Fig. 3a) we used different sets of simulations to generate data from which we could estimate the persistent subspace (as explained above), and to analyse network dynamics when initialized within these subspaces. In all cases, for a fair comparison, the magnitude of initial conditions was fixed (Nonlinear networks with instantaneous inputs, De novo linear networks), and only their direction was affected by constraining them to one of these subspaces.

\section{Measures of subspace overlap}

In order to measure the overlap of high dimensional neural dynamics with the subspaces we identified, we used one of two methods. First, for analysing network dynamics across two conditions chosen to correspond to 'opposite' stimulus cues (Fig. 1e,j, Fig. 2c,d,e, Extended Data Fig. 1c,d,g,h, Extended Data Fig. 2c,d, and Extended Data Fig. 4), such that the coding part of the persistent subspace was one-dimensional, we simply measured the projection of neural dynamics onto the first eigenvector (i.e. the eigenvector associated with the largest real eigenvalue) of the corresponding subspace using a dot product:

$$
\text { activity along mode }(t)=\mathbf{u}^{\top} \mathbf{x}(t)
$$

where u may correspond to the most persistent, or the most amplifying mode, or the first PC of the persistentorthogonalized cue subspace (as defined above). We also used the same measure for visualising the quality of fit of linear neural network dynamics to experimental data (Fitting linear neural networks to neural responses) with $\mathbf{u}$ being the first PC of the full state space of neural firings rates (Fig. 4e). In those cases, when $\mathbf{u}$ had to be estimated from neural responses (Fig. 1e,j, Fig. 4e, Extended Data Fig. 1c,d,g,h), we used a cross-validated approach, using different subsets of the data to determine $\mathbf{u}$ and $\mathbf{x}(t)$ (from a single split of the data). In other cases, $\mathbf{u}$ was determined from the truly deterministic dynamics of the system and thus there was no need for cross-validation.

Second, to measure subspace overlaps for $d$-dimensional neural activities across multiple conditions and time points within coarser time bins (Fig. 3c, Fig. 4f, Fig. 5e, Extended Data Fig. 3d, Extended Data Fig. 5a-d; bottom, Extended Data Fig. 5f, Extended Data Fig. 6c, Extended Data Fig. 7d, and Extended Data Fig. 8d, and Extended Data Fig. 10b,c,f,g), thus corresponding to high-dimensional coding sub-spaces, we used the following properly normalized measure:

$$
\text { variance explained }\left(t, t^{\prime}\right)=\frac{\operatorname{Tr}\left(\mathbf{U}^{\top}\left(t^{\prime}\right) \mathbf{\Sigma}(t) \mathbf{U}\left(t^{\prime}\right)\right)}{\operatorname{Tr}\left(\mathbf{P}^{\top}(t) \mathbf{\Sigma}(t) \mathbf{P}(t)\right)}
$$

where $\boldsymbol{\Sigma}(t)$ is the covariance matrix of neural activities across conditions and raw (1-ms) time points within time bin $t$, the columns of $\mathbf{P}(t)$ are the first principal components of neural activities within time bin $t$ (i.e. the eigenvectors of $\boldsymbol{\Sigma}(t)$ associated with the largest eigenvalues), and $\mathbf{U}\left(t^{\prime}\right)$ is the subspace of interest with respect to which overlaps are computed (which itself may or may not depend on time, see below). The time resolution of $t$ and $t^{\prime}$ (i.e. the duration of time bins within which data was used to compute the corresponding terms at a given $t$ or $t^{\prime}$ ), the 
bioRxiv preprint doi: https://doi.org/10.1101/2021.11.16.468360; this version posted December 17, 2021. The copyright holder for this preprint (which was not certified by peer review) is the author/funder, who has granted bioRxiv a license to display the preprint in perpetuity. It is made available under aCC-BY 4.0 International license.

choice of $\mathbf{U}\left(t^{\prime}\right)$, and the number of vectors used for constructing $\mathbf{U}\left(t^{\prime}\right)$ and $\mathbf{P}(t)$ depended on the analysis (see below).

Specifically, for measuring subspace overlap between neural activity and persistent vs. amplifying modes (Fig. 3c, Fig. 4f, Fig. 5e, Extended Data Fig. 3d, Extended Data Fig. 5f, and Extended Data Fig. 10f,g), we set $\mathbf{U}\left(t^{\prime}\right)=\mathbf{U}$ where the columns of $\mathbf{U}$ are the first $d / 4$ eigenvectors of the most persistent or amplifying subspace (orthogonalized using a QR decomposition for the most persistent modes - this was not necessary for most amplifying modes which are orthogonal by construction), or $d / 4$ randomly chosen orthonormal vectors as a control (shown as 'chance'; computed analytically as $1 / 4$ for 'de novo' linear networks, Fig. $3 c$ and Extended Data Fig. 5f, and numerically for fitted linear networks, also yielding values of approximately $1 / 4$, Fig. 4f, Fig. 5e, Extended Data Fig. 3d, and Extended Data Fig. 10f). $\mathbf{P}(t)$ contained the first $d / 4$ principal components. In this case, a value of 1 for this metric implies that the $d / 4$ directions of greatest variability in neural activity overlap exactly with the $d / 4$-dimensional subspace spanned by $\mathbf{U}$. The time resolution of $t$ was $20 \mathrm{~ms}$ (for clarity, bins to be plotted were subsampled in the corresponding figures). Note that when this analysis was performed on linear networks fitted to neural data (experimentally recorded or simulated), $\mathbf{U}, \mathbf{P}(t)$, and $\boldsymbol{\Sigma}(t)$ were all obtained from the same fitted linear network (i.e. no cross-validation). Specifically the parameters of the network were used to determine $\mathbf{U}$ (see Fitting linear neural networks to neural responses), and the neural responses these fitted linear dynamics generated (rather than the original neural responses that were fit by the linear model) were used to determine $\boldsymbol{\Sigma}(t)$ and thus $\mathbf{P}(t)$. See Statistics for computing the significance of these overlaps (and their differences).

For analyzing subspace sharing between different task epochs (Extended Data Fig. 5a-d; bottom, Extended Data Fig. 6c, Extended Data Fig. 7d, Extended Data Fig. 8d, and Extended Data Fig. 10b), U( $\left.t^{\prime}\right)$ contained the top $k$ principal components (PCs) of neural activity within the time bin indexed by $t^{\prime}$ (we used $k=10$ for the monkey data and $k=4$ for our models because the models typically exhibited lower dimensional dynamics), while $\mathbf{P}(t)$ included all PCs within the time bin indexed by $t$. For these, we performed principal components analysis with dimensions corresponding to neurons and data points corresponding to time points and cue conditions. The time resolution of both $t$ and $t^{\prime}$ was $250 \mathrm{~ms}$, such that the time periods (relative to cue onset) that we used were -500 to $-250 \mathrm{~ms}$ (spontaneous epoch), 0 to 250 (cue epoch), 1250 to $1500 \mathrm{~ms}$ (delay epoch), and the first $250 \mathrm{~ms}$ after the go cue, i.e. $t_{\mathrm{go}}$ to $t_{\mathrm{go}}+0.25 \mathrm{~s}$ (go epoch). In this case, $\mathbf{U}\left(t^{\prime}\right), \mathbf{P}(t)$ and $\boldsymbol{\Sigma}(t)$ were obtained by fitting all the available neural data (i.e. no cross-validation). See also Ref. 64 for an 'alignment index' metric that is closely analogous to this use of this metric, but uses $\mathbf{U}\left(t^{\prime}\right)$ and $\mathbf{P}(t)$ that contain the same number of eigenvectors, as in our previous case, and are estimated in a cross-validated way, using different sets of trials.

For showing how much variance the top 2 delay epoch PCs capture over time (Extended Data Fig. 10c), we set $\mathbf{U}\left(t^{\prime}\right)=\mathbf{U}$ where the columns of $\mathbf{U}$ are the first 2 principal components of neural activities over the time period 750 to $250 \mathrm{~ms}$ before the go cue, i.e. $t_{\mathrm{go}}-0.75$ to $t_{\mathrm{go}}-0.25 \mathrm{~s}$, and $\mathbf{P}(t)$ also includes the top 2 principal components. The resolution for $t$ was $10 \mathrm{~ms}$ (for clarity, bins to be plotted were subsampled in the corresponding figure). In this case, we estimated $\mathbf{U}$ and $\mathbf{P}(t)$ in a cross-validated way-we estimated $\mathbf{U}$ using training data and $\mathbf{P}(t)$ and $\boldsymbol{\Sigma}(t)$ using test data, and we show results averaged over 10 random 1:1 train:test splits of the data. See also Ref. 11 for a measure that is closely related to this use of this metric, but uses the number of neurons in the denominator instead of the total variance.

\section{Linear decoding}

We fitted decoders using linear discriminant analysis to decode the stimulus cue identity from neural firing rates (Fig. 1f,k, Fig. 3a,b, Fig. 4b,c, Fig. 5c,d, Extended Data Fig. 3c, Extended Data Fig. 5a-e, Extended Data Fig. 6a,b, Extended Data Fig. 7b,c, Extended Data Fig. 8b,c, and Extended Data Fig. 10a). We constrained the decoders to be 2-dimensional (in line with previous studies ${ }^{11}$ ) because this was a sufficient dimensionality to decode responses. (We also trained decoders using logistic regression in the full activity space and obtained qualitatively similar results; not shown.) We primarily considered two types of decoding analyses: we either trained decoders on late delay activity and tested on all time points ('delay-trained decoder', e.g. Fig. 3a), or we trained decoders separately at every time point and tested on all times ('full cross-temporal decoding', e.g. Fig. 3b). In all cases, we measured decoding performance in a cross-validated way, using separate sets of neural trajectories to train and test the decoder, and we show results averaged over 10 random 1:1 train:test splits of the data. For delaytrained decoders, training data consisted of pooling neural activity over a $500 \mathrm{~ms}$ time interval (the time interval is shown by a horizontal black bar in all relevant figures), and tested the thus-trained decoder with data in each $1 \mathrm{~ms}$ time bins across the trial (for clarity, test bins to be plotted were subsampled every $10 \mathrm{~ms}$ in the corresponding figures). For full cross-temporal decoding, we binned neural responses into $10 \mathrm{~ms}$ time bins and trained and tested on all pairs of time bins (specifically, we plotted mean decoding performance across the 101-ms raw time bins corresponding to each 10 -ms testing bin). We used a shrinkage (inverse regularisation parameter on the Euclidean norm of decoding coefficients) of 0.5 for all main figures (we also tested various other values and found 
bioRxiv preprint doi: https://doi.org/10.1101/2021.11.16.468360; this version posted December 17, 2021. The copyright holder for this preprint (which was not certified by peer review) is the author/funder, who has granted bioRxiv a license to display the preprint in perpetuity. It is made available under aCC-BY 4.0 International license.

qualitatively similar results; not shown). Chance level decoding was defined as $1 / C$, where $C=2$ or 6 is the number of cue conditions that need to be decoded (Tables 1 and 3).

\section{Quality of fit for linear models fitted to neural responses}

When fitting linear models to neural data (experimentally recorded or simulated; Fitting linear neural networks to neural responses) we used a cross-validated approach for measuring the quality of our fits, with a random 1:1 train:test split of the data (Fig. 4d). For this, we first fitted the model on training data $\left(\mathbf{x}_{*}^{(c)}=\mathbf{x}_{\text {train }}^{(c)}\right.$ in Eq. 7). The quality of fit was then computed on the test data, $\mathbf{x}_{\text {test }}^{(c)}$, as the fraction of variance of $\mathbf{x}_{\text {test }}^{(c)}(t)$ explained by the simulated responses (after the appropriate projection, i.e. $\mathbf{C} \mathbf{x}^{(c)}(t)$ ), across all 20 dimensions weighted by $\mathbf{D}$ (all parameters, including $\mathbf{P}, \mathbf{C}$ and $\mathbf{D}$, were set to their values obtained by fitting the training data). In other words, we computed the Pearson $R^{2}$ with respect to the identity line using the mean squared error, $\varepsilon^{2}$ in Eq. 6 , with the momentary error in Eq. 7 computed using $\mathbf{x}_{*}^{(c)}=\mathbf{x}_{\text {test }}^{(c)}$. Once the quality of fit for this split was thus established, we conducted all further analysis involving fitted linear models with the model that was fit to the training half of this split.

As a meaningful lower bound on our quality of fit measure, we also computed the same measure (i.e. fitting a linear dynamical system to training data and calculating the quality of fit using test data) for 100 different timeshuffled controls of the original train:test split of the data (Fitting linear neural networks to neural responses), such that we shuffled time bins coherently between the training and the test data, across neurons and conditions (Fig. 4d, dark grey).

To calibrate how much our fits were limited by the noisiness of the data, we also computed the quality of fit directly between $\mathbf{x}_{\text {train }}^{(c)}(t)$ and $\mathbf{x}_{\text {test }}^{(c)}(t)$ (i.e. using the mean squared error, $\varepsilon^{2}$ in Eq. 6, with the momentary error redefined as $\left.\mathbf{e}^{(c)}(t)=\mathbf{x}_{\text {train }}^{(c)}(t)-\mathbf{x}_{\text {test }}^{(c)}(t)\right)$ for 100 random 1:1 train:test splits of the data (Fig. 4d, light grey). The extent to which the $R^{2}$ computed with this control was below 1 reflected the inherent (sampling) noise of the experimental data that limited the quality of fit obtainable with any parametric model, including ours that was based on linear dynamics. Moreover, a cross-validated $R^{2}$ computed with our fits that was higher than the $R^{2}$ obtained with this control (Fig. 4d dark and light blue vs. light grey) meant that the inherent assumption of linear dynamics in our model acted as a useful regularizer to prevent the overfitting that this overly flexible control inevitably suffered from. See more in Statistics on statistical testing for our quality of fit measure.

When fitting to simulated neural data, we obtained high quality of fits using the same measure $\left(R^{2}>0.95\right.$, not shown).

\section{Overlap between the coding populations during the cue and delay epochs}

To test whether separate neural populations encode stimulus information during the cue and delay epochs (Extended Data Fig. 10e), we trained (non-cross validated) decoders to decode cue identity using logistic regression on either cue-epoch activity ('cue-trained'; the first $250 \mathrm{~ms}$ of activity after cue onset) or delay-epoch activity ('delay-trained'; 1250-1500 ms after cue onset). We used an L2 regularisation penalty of 0.5 (we also tested other regularisation strengths and observed no substantial changes in our results). We took the absolute value of decoder weights as a measure of how strongly neurons contributed to decodability (either positively or negatively). We then binarized the absolute 'cue-trained' and 'delay-trained' weights using their respective median values as the binarization threshold. (This binarization reduces a potential bias effect from large or small weight values in our analysis.) Our measure of overlap between the coding populations during the cue and delay epochs, was then simply the inverse normalized Hamming distance between these two sets of binarized weights:

$$
\text { overlap }=1-\left\langle\left|w_{n, c}^{\text {cue }}-w_{n, c}^{\text {delay }}\right|\right\rangle_{n, c}
$$

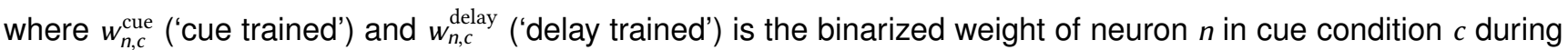
the cue and delay epochs, respectively, and $\langle\cdot\rangle_{n, c}$ denotes taking the mean across neurons and cue conditions. For completely overlapping populations, this measure takes a values of 1 , for completely non-overlapping populations, it takes a values of 0 , and for random overlap (shown as 'chance') it takes a values of 0.5 .

For the shuffle controls, we randomly permuted the neuron indices of the delay-trained weights (such that using the median as a threshold thus resulted in values close to 0.5, i.e. chance level; Extended Data Fig. 10e). We show results (for both the original analysis and shuffle control) for 10 random halves of the data (equivalent to the training halves of 10 different $1: 1$ train:test splits). We also tested a variety of percentile values other than the median and our results did not change substantially (choosing a threshold other than the median causes both the data and shuffle controls to have overlap values lower than those that we obtained with the median as the threshold, but it does not substantially affect the difference between them). As an additional control, we also 
bioRxiv preprint doi: https://doi.org/10.1101/2021.11.16.468360; this version posted December 17, 2021. The copyright holder for this preprint (which was not certified by peer review) is the author/funder, who has granted bioRxiv a license to display the preprint in perpetuity. It is made available under aCC-BY 4.0 International license.

removed neurons that did not contribute to decodability: we removed neurons that had a thresholded weight of 0 for all 6 cue conditions in both the cue and delay epochs. This resulted in removing 13.3 neurons on average for monkey $\mathrm{K}$ and 33.5 neurons for Monkey $\mathrm{T}$ (when using the median as the threshold) and our results did not change substantially (not shown).

\section{Finding fixed points}

For finding the fixed points of nonlinear network dynamics (Fig. 1e,j, Extended Data Fig. 1b,f, and Extended Data Fig. 6d), we used a slow-point analysis method ${ }^{30}$ that searches for an $\mathbf{x}$ for which the $\mathrm{L} 2$ norm of the gradient determined by the autonomous dynamics of the network is below a threshold. Note that this was only possible in model neural networks as the method requires access to the equations (and parameters) defining the true (nonlinear) dynamics of a system.

Specifically, for network dynamics governed by (cf. Eqs. 1 and 2)

$$
\frac{\mathrm{d} \mathbf{x}(t)}{\mathrm{d} t}=\psi(\mathbf{x}(t))
$$

for some function $\psi$, we sought to find points $\mathbf{x}^{*}$ such that $\left\|\psi\left(\mathbf{x}^{*}\right)\right\|_{2}$ is small. To achieve this, we drew 1000 $\mathbf{x}$ 's from a spherical Gaussian distribution with mean 0 and variance 10 (the large variance helps to ensure that we cover a large part of state space) and we optimized each $\mathbf{x}$ to minimize $\|\boldsymbol{\psi}(\mathbf{x})\|_{2}$ using gradient descent with gradients obtained by back-propagation with an Adam optimizer ${ }^{80}$. We used an adaptive learning rate (which we found worked substantially better than a fixed learning rate in this scenario) that started at 0.1 and halved every 1000 training iterations (we used 5000 training iterations in total). Finally, we identified the x's obtained at the end of optimization as stable fixed points, $\mathbf{x}^{*}$, if $\|\boldsymbol{\psi}(\mathbf{x})\|_{2}<0.001$ and if the largest real part in the eigenvalues of the linearization of $\psi(\mathbf{x})$ around $\mathbf{x}^{*}$ was less than 0 .

\section{Correlations between initial and final neural firing rates}

To measure correlations between initial and final simulated activities, we used the Pearson correlation coefficient (with respect to the identity line) between initial and final mean-centered firing rates across neurons within the same simulation (i.e. no cross-validation; Fig. 1c,h; insets). Histograms show the distribution of this correlation across 6 cue conditions (and the 10 different networks, each simulated 10 times, see above) using a kerneldensity estimate (Fig. 1d,e and Extended Data Fig. 1c,d,g,h).

\section{Statistics}

We performed statistical hypothesis testing in two cases.

First, we tested whether the quality of fit of linear models to experimental data was sufficiently high using permutation tests. To construct the distribution of our test statistic (cross-validated $R^{2}$, see also Quality of fit for linear models fitted to neural responses) under the null hypothesis, we used $n=100$ different random time shuffles of the data (Fig. 4d, dark grey), such that we shuffled time bins coherently between the training and the test data, across neurons and conditions, and for each shuffle used the same random 1:1 train:test split as for the original (unshuffled) data. For additional calibration, we also constructed the distribution of our test statistic under the alternative hypothesis that all cross-validated errors were due to sampling noise differences between the train and test data. For this, we used $n=100$ random 1:1 train:test splits of the (original, unshuffled) data, and measured the quality of fit directly between the test data and the training data (rather than a model fitted to the training data, see also Quality of fit for linear models fitted to neural responses; Fig. 4d, light grey). In both cases, we computed the two-tailed p-value of the test statistic as computed on the real data (Fig. $4 \mathrm{~d}$, blue lines) with respect to the corresponding reference distribution.

Second, we also used a permutation test-based approach to test whether the experimentally observed overlaps with persistent and amplifying modes (or their differences) were significantly different from those expected by chance. For testing the significance of overlaps in a given time step, we constructed the distribution of our test statistics (the overlap measures; Measures of subspace overlap) under the null hypothesis by generating $n=200$ random subspaces within the space spanned by the 20 PCs we extracted from the data (Fitting linear neural networks to neural responses), dimensionality matched to the persistent and amplifying subspaces (i.e. 5 orthogonal dimensions), and computed the same subspace overlap measures for the data in the given time step with respect to these random subspaces (Fig. 4f and Extended Data Fig. 10f-g; grey line and shading). For testing the significance of differences between overlaps (amplifying vs. persistent at a given time step, or amplifying or persistent between two different time steps), our test statistic was this difference (i.e. a paired test), and our null distribution was constructed by measuring it for $n=200$ pairs of random subspace overlaps at the 
appropriate time step(s). Once again, in all these cases we computed the two-tailed p-value of the test statistic as computed on the real data (Fig. $4 \mathrm{f}$ and Extended Data Fig. 10f-g, green and red lines) with respect to the corresponding reference distribution.

Note that we did not compute $p$-values across multiple splits of the data because this led to $p$-value inflation as we increased the number of splits. Instead, we repeated all relevant analyses on 10 different random 1:1 train:test splits to see if our results were robust to the choice of data split. Indeed, we obtained qualitatively and quantitatively (in terms of $p$-values for quality of fits, and overlaps) similar results for all these splits.

Permutation tests do not assume that the data follows any pre-defined distribution. No statistical methods were used to predetermine experimental sample sizes. Sample sizes for permutation tests ( $n$ above) were chosen so as to be able to determine $p$-values to a precision of 0.02 (quality of fits) or 0.01 (subspace overlaps).

\section{References}

75. Watanabe, K. \& Funahashi, S. Neural mechanisms of dual-task interference and cognitive capacity limitation in the prefrontal cortex. Nature Neuroscience 17, 601-611 (2014).

76. Watanabe, K. \& Funahashi, S. A dual-task paradigm for behavioral and neurobiological studies in nonhuman primates. Journal of Neuroscience Methods 246, 1-12 (2015).

77. Drucker, C. B., Carlson, M. L., Toda, K., DeWind, N. K. \& Platt, M. L. Non-invasive primate head restraint using thermoplastic masks. Journal of Neuroscience Methods 253, 90-100 (2015).

78. Ninomiya, T., Dougherty, K., Godlove, D. C., Schall, J. D. \& Maier, A. Microcircuitry of agranular frontal cortex: contrasting laminar connectivity between occipital and frontal areas. Journal of Neurophysiology 113, 3242-3255 (2015).

79. Hennequin, G., Ahmadian, Y., Rubin, D. B., Lengyel, M. \& Miller, K. D. The Dynamical Regime of Sensory Cortex: Stable Dynamics around a Single Stimulus-Tuned Attractor Account for Patterns of Noise Variability. Neuron 98, 846-860.e5 (2018).

80. Kingma, D. P. \& Ba, J. L. Adam: A method for stochastic optimization. arXiv 1412.6980 (2014). 
bioRxiv preprint doi: https://doi.org/10.1101/2021.11.16.468360; this version posted December 17, 2021. The copyright holder for this preprint (which was not certified by peer review) is the author/funder, who has granted bioRxiv a license to display the preprint in perpetuity. It is made available under aCC-BY 4.0 International license.

\section{Extended data figures}

a
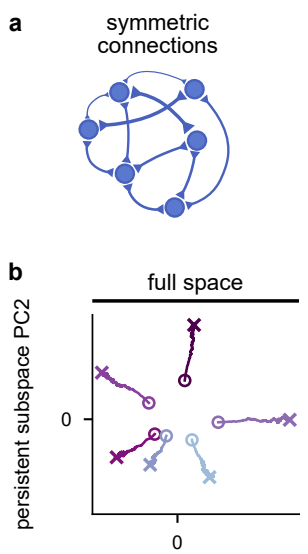

persistent subspace $\mathrm{PC} 1$
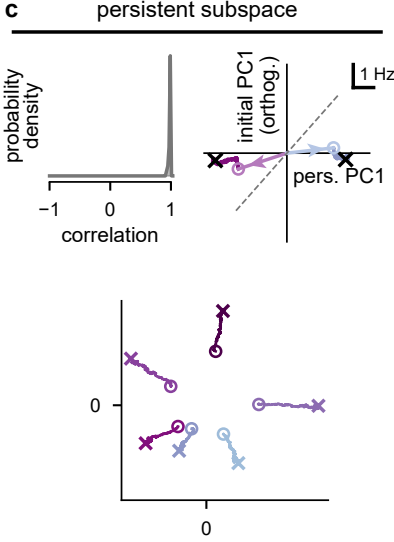

persistent subspace PC1
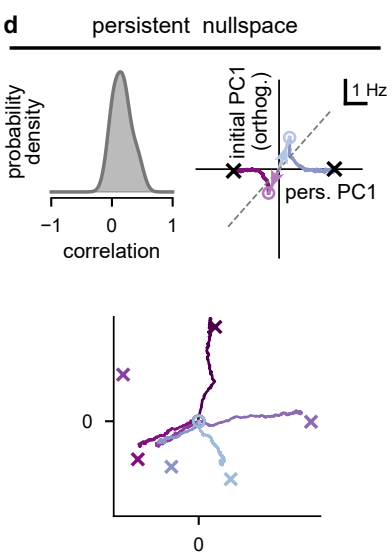

persistent subspace $\mathrm{PC} 1$
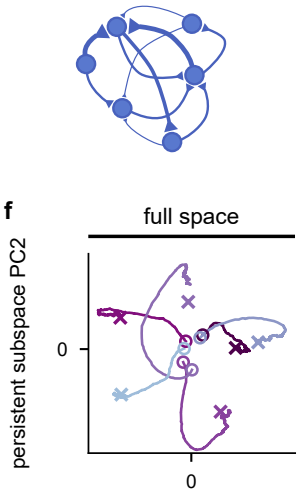

persistent subspace PC1

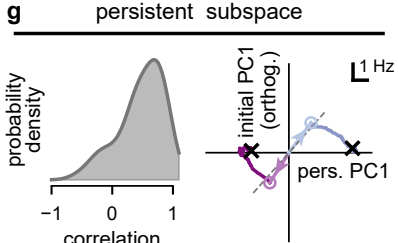

correlation

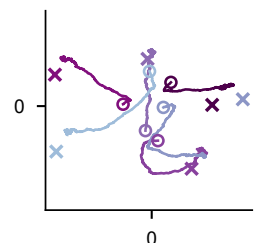

persistent subspace $\mathrm{PC} 1$
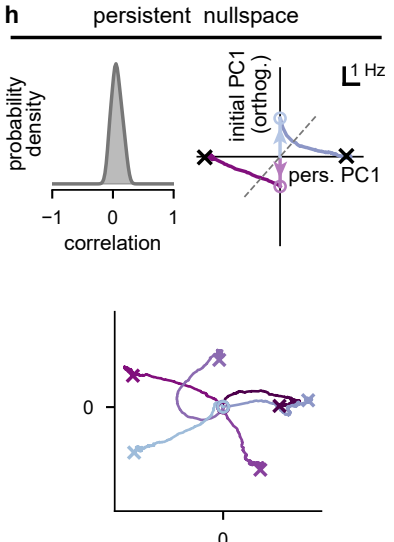

persistent subspace $\mathrm{PC} 1$

Extended Data Fig. 1 | Attractor network dynamics with or without constraints on the initial condition of the dynamics. a, Illustration of an attractor network with symmetric connections. b-d, Analysis of neural responses in symmetric attractor networks (such as shown in a). b, Sub-threshold activity for all 6 cue conditions (color trajectories) with initial conditions optimized within the full state space (Methods). Open circles show the optimized initial conditions and crosses show stable fixed points. We show neural activity projected onto the top two principal components of the persistent subspace. c. Analysis of neural responses when initial conditions are constrained to lie within the 5-dimensional persistent subspace. Top left: distribution of Pearson correlations between initial and final mean-centered neural firing rates across all 6 cue conditions and 10 networks (same as Fig. 1d, but for persistent subspace-constrained inputs, corresponding to green line in Fig. 1f). Top right: sub-threshold activity for 2 cue conditions in an example network (color trajectories; same as Fig. 1e, but for persistent subspace-constrained inputs, corresponding to green line in Fig. 1f). Open circles (with arrows pointing to them from the origin) show the optimized initial conditions, black crosses show stable fixed points, dashed grey line is the identity line. Horizontal axis (persistent PC1) shows neural activity projected on to the 1st principal component (PC1) of network activities at the end of the delay period (across the 2 conditions shown), vertical axis (initial PC1 (orthogonalized)) shows projection to PC1 of initial neural activities orthogonalized to persistent PC1. Bottom: same as $\mathbf{b}$, but for persistent subspace-constrained inputs, corresponding to green line in Fig. 1f. d, Same as c, but for persistent nullspace-constrained inputs. Note that the distribution of Pearson correlations of neural firing rates (top left) is distinct from a delta function at 0 because we constrained the initial conditions in the space of sub-threshold activities (rather than firing rates). In the bottom panel, which shows sub-threshold activity, we see that indeed all the colored circles overlap at the origin. e, Illustration of an attractor network without a symmetric connectivity constraint. $\mathbf{f}-\mathbf{h}$, Same as $\mathbf{b}-\mathbf{d}$ but for attractor networks without a symmetric connection constraint (i.e. panels $f, g$, and $h$, respectively correspond to the networks shown by the black, green, and red lines in Fig. 1k). 
bioRxiv preprint doi: https://doi.org/10.1101/2021.11.16.468360; this version posted December 17, 2021. The copyright holder for this preprint (which was not certified by peer review) is the author/funder, who has granted bioRxiv a license to display the preprint in perpetuity. It is made available under aCC-BY 4.0 International license.
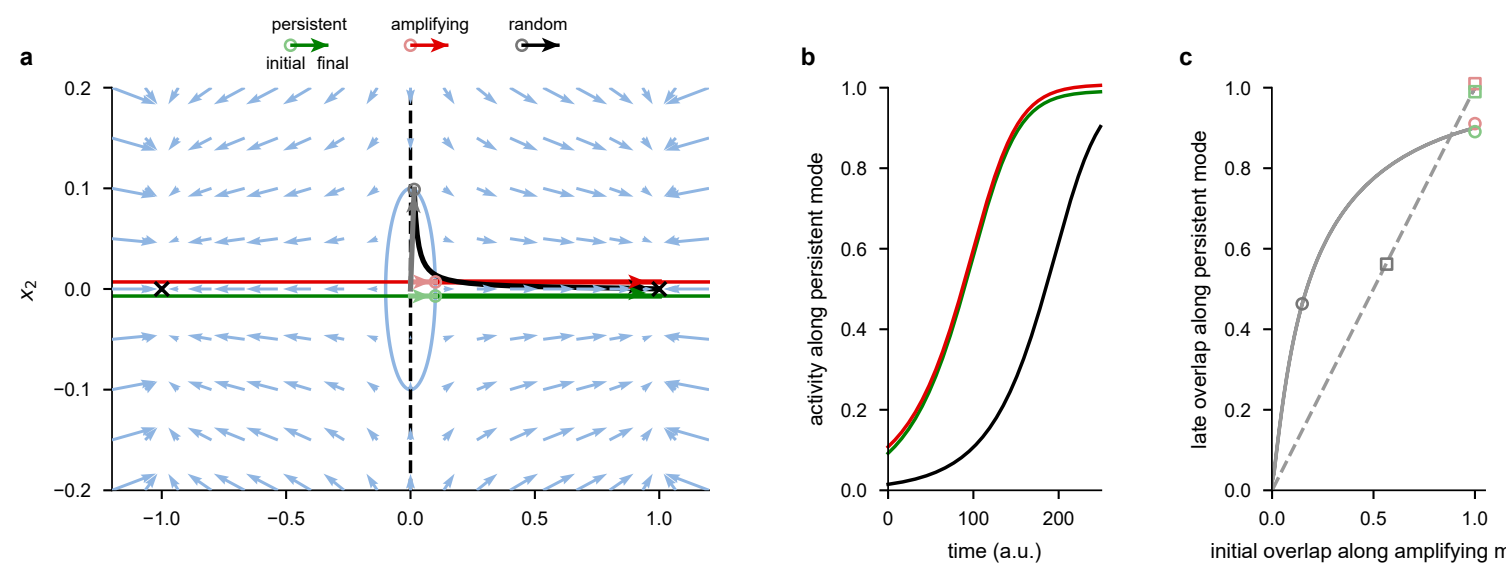

initial overlap along amplifying mode
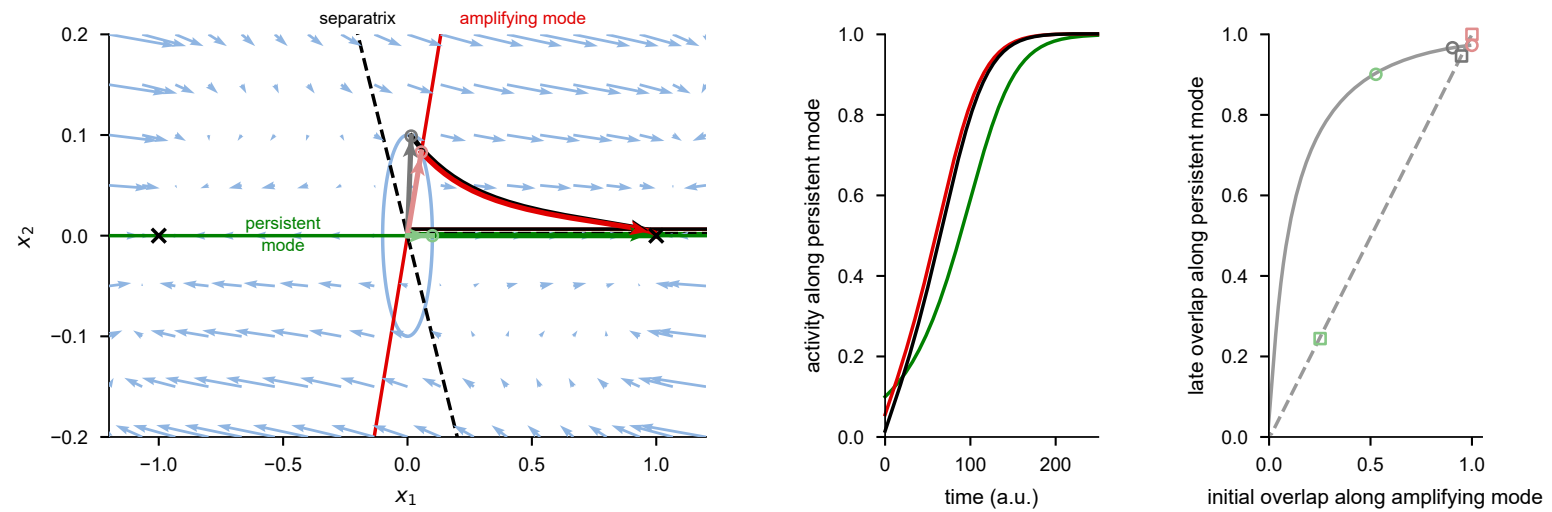

Extended Data Fig. 2 | Analysis of canonical nonlinear attractor systems. a, State space of a canonical nonlinear system with two attractors and a symmetric (top) and non-symmetric Jacobian (bottom, see also Methods, Supplementary Math Note; cf. Fig. 2b). Pale blue arrows show flow field dynamics (direction and magnitude of movement in the state space as a function of the momentary state). Black crosses indicate asymptotically stable fixed points (i.e. attractor states), dashed black line shows the separatrix (the manifold separating the basins of attraction of the two attractors). Thin green and red lines indicate the locally most persistent and amplifying modes around the origin, respectively (lines are offset slightly in the top panel to aid visualisation). Pale green, red, and grey arrows with open circles at the end indicate most persistent, amplifying, and random initial conditions, respectively. Blue ellipses show the fixed initial condition norm around the origin to highlight the different axis scales. Dark green, red, and black arrows show neural dynamics starting from the corresponding initial condition. b, Time course of dynamics of the system along the persistent mode (i.e. the projection onto the green line in a) when started from the persistent (green), most amplifying (red), or random (black) initial conditions for the symmetric (top) and the unconstrained system (bottom). c, Late overlap with the locally persistent mode as a function of initial overlap with the locally most amplifying mode in the canonical nonlinear systems shown in panels $\mathbf{a}-\mathbf{b}$ (solid grey line) and, for comparison, in the linear networks of Fig. 2a-c (dashed grey line) for symmetric (top) or non-symmetric/unconstrained systems (bottom). Late overlap is measured as the mean overlap of activity along the persistent mode (panel b, from $t=100$ to $t=250$ for the canonical nonlinear system; Fig. 2c, from $t=0.8 \mathrm{~s}$ to $t=2 \mathrm{~s}$ for the linear networks). Open circles and squares indicate the random (grey), persistent (pale green), and most amplifying (pale red) initial conditions used respectively in panels $\mathbf{a}$ and $\mathbf{b}$ for the canonical nonlinear system, and in Fig. $2 \mathrm{~b}-\mathrm{c}$ for the linear networks. 
bioRxiv preprint doi: https://doi.org/10.1101/2021.11.16.468360; this version posted December 17, 2021. The copyright holder for this preprint (which was not certified by peer review) is the author/funder, who has granted bioRxiv a license to display the preprint in perpetuity. It is made available under aCC-BY 4.0 International license.
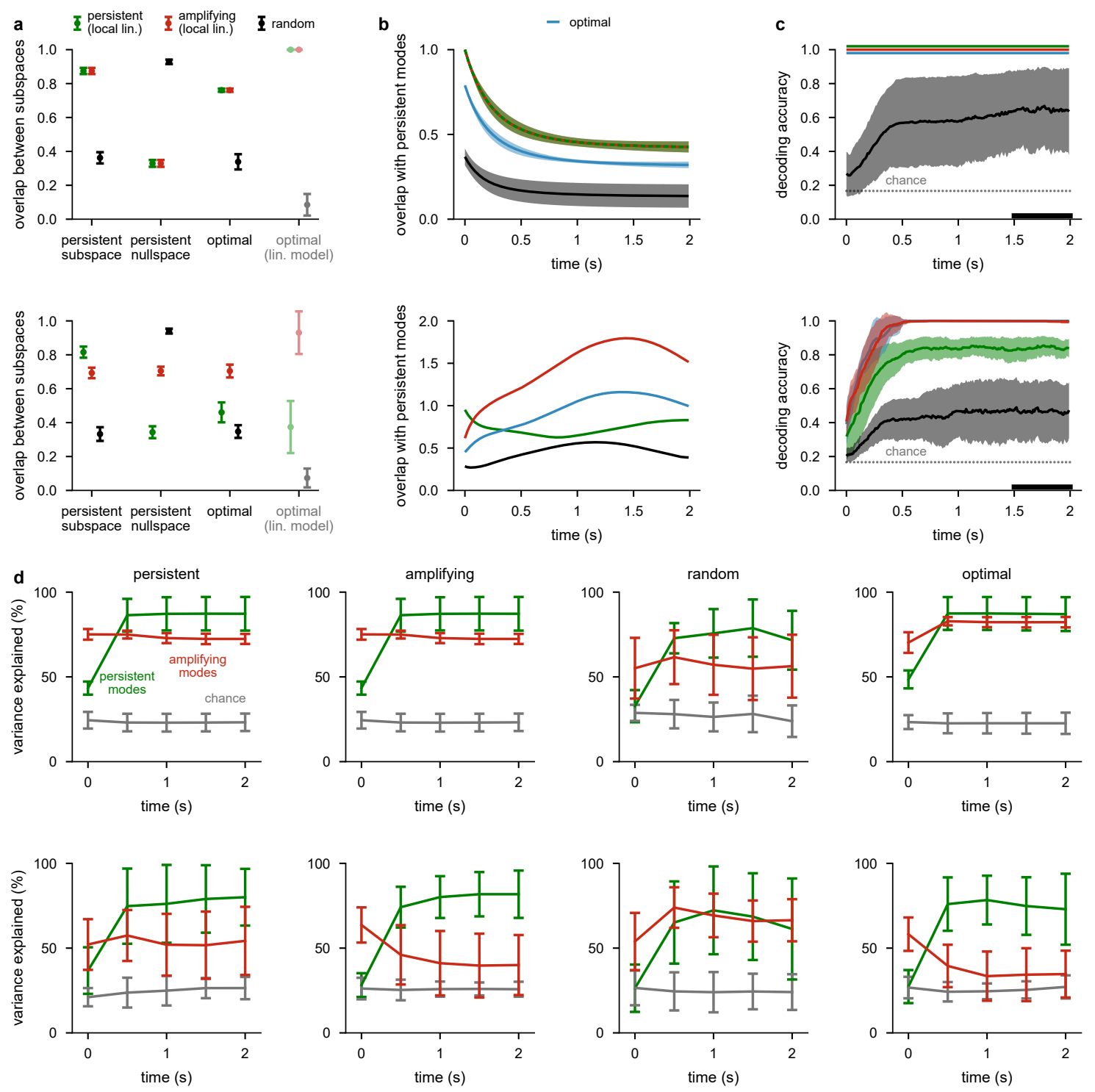

Extended Data Fig. 3 | Linear analyses of the nonlinear attractor networks of Fig. 1. a, Overlap (mean \pm 1 s.d. across 10 networks) of the 5 locally most persistent (green), most amplifying (red), or random directions (black) of the symmetric (top) and unconstrained (bottom) networks from Fig. 1, obtained using a local linearization around the origin, with the 'persistent subspace' and 'persistent nullspace' of the original non-linear dynamics, obtained without linearization (as used in Fig. $1 \mathrm{f}$ and $\mathrm{k}$, red and green), and the 5-dimensional subspace spanned by the 6 'optimal' initial conditions of the original nonlinear dynamics (used in Fig. 1c-e, h-j, and f and k, black). For comparison, we also show the overlap (mean \pm 1 s.d. across 100 networks) of the single most persistent (pale green), most amplifying (pale red), and random (grey) direction with the optimal initial condition of the linear networks from Extended Data Fig. 4c,d ('optimal (lin. model)').

(Caption continued on next page.) 
bioRxiv preprint doi: https://doi.org/10.1101/2021.11.16.468360; this version posted December 17, 2021. The copyright holder for this preprint (which was not certified by peer review) is the author/funder, who has granted bioRxiv a license to display the preprint in perpetuity. It is made available under aCC-BY 4.0 International license.

Extended Data Fig. 3 | Linear analyses of the nonlinear attractor networks of Fig. 1 (cont'd). b, Time course of the overlap (mean \pm 1 s.d. across 10 networks, s.d. not shown in bottom for visual clarity) of the linearized dynamics of symmetric (top) and unconstrained networks (bottom) with the subspace spanned by their most persistent modes when started from initial conditions that were optimized for the decoding accuracy of the nonlinear dynamics while constrained to be within the locally most persistent (green), most amplifying (red), or a random subspace (black). The linear dynamics, the persistent subspace wrt. which overlap is measured, and the subspaces within which initial conditions were constrained while being optimized, were all based on a local linearization of the nonlinear dynamics around the origin. Compare with Fig. $2 \mathrm{e}$ for the analogous plots for linear networks. For reference, blue line shows overlap of the same linearized dynamics when started from the initial conditions directly optimized for the decoding accuracy of the nonlinear dynamics without subspace constraints (used in Fig. 1c-e, h-j, and f and k, black). For consistency with Fig. 2b-e (where initial conditions were constrained to have unit norm), we scaled activity by the norm of the initial condition (which was constrained to be 3 here; Methods). c, Performance (mean \pm 1 s.d. across 10 networks) of a delay-trained decoder (black bar indicates decoder training time period; Methods) on neural activity in stochastic nonlinear symmetric (top) and unconstrained networks (bottom) over time. Colors indicate initial conditions as in $\mathbf{b}$. (Blue line shows same data as black line in Fig. $1 \mathrm{f}$ and k). Grey dotted line shows chance level decoding. Green, red, and blue lines are vertically offset slightly in the top panel to aid visualization. Compare with Fig. $3 a$ (noise matched) for the analogous plots for linear networks (though with non-instantaneous inputs). d, Percent variance explained (mean \pm 1 s.d. across 10 networks) by the subspace spanned by either the $25 \%$ (i.e. 5) most persistent (green) or $25 \%$ (i.e. 5) most amplifying (red) modes as a function of time for 20-dimensional linear dynamical systems fitted to the neural responses generated by the symmetric (top) and unconstrained (bottom) nonlinear networks when started from the same (optimized) initial conditions analyzed in b-c: constrained to be within the locally most persistent (far left), most amplifying (center left), or a random subspace (center right), as determined by the local linearization of the dynamics, or without subspace constraints (far right). Grey lines show chance level overlap defined as the expected overlap with a randomly chosen subspace occupying $25 \%$ of the full space (i.e. 5 dimensions). Compare with Fig. $3 c$ for the analogous plots for linear networks (though with non-instantaneous inputs, and performance-matched levels of noise, see also Supplementary Math Note) and with Fig. $4 \mathrm{f}$ and Extended Data Fig. 10f,g for analogous plots of linear dynamical systems fitted to experimental data.
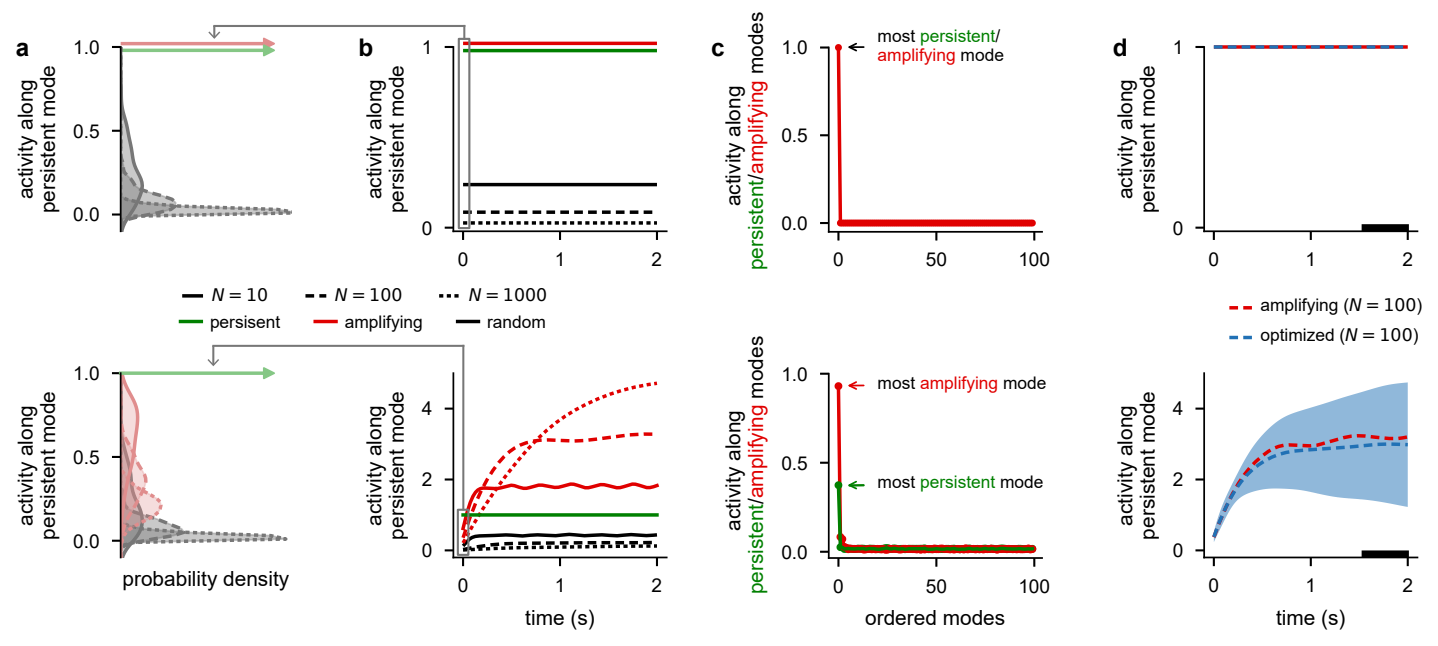

Extended Data Fig. 4 | Analysis of linear networks of different sizes. a, Distributions of absolute overlap with the persistent mode for persistent (pale green), most amplifying (pale red), or random initial conditions (grey) across 100 randomly sampled linear symmetric (top) and unconstrained networks (bottom) consisting of either 10 (solid), 100 (dashed), or 1000 (dotted) neurons (cf. Fig. 2d). The persistent initial conditions produced delta functions at 1 (arrows). Results for persistent and most amplifying initial conditions are identical in symmetric networks (top). b. Time course of mean (across the 100 networks from a) absolute overlap with the persistent mode when starting network dynamics from persistent (green), most amplifying (red), or random initial conditions (black) in symmetric (top) and unconstrained networks (bottom) consisting of either 10 (solid), 100 (dashed), or 1000 (dotted) neurons (cf. Fig. 2e). Results for persistent and most amplifying initial conditions are identical in symmetric networks (top). c, Mean (across 100 networks) overlap of initial conditions that were optimized so as to generate persistent activity in 100-neuron noisy symmetric (top) and unconstrained (bottom) networks with 100 orthogonal modes ordered by their persistence (green) or amplification (red) (i.e. corresponding to the rank ordered eigenvectors of the weight matrix, green, or of the observability Gramian of the dynamics, red; Methods). In symmetric networks (top), the optimized initial conditions overlap only with the most amplifying mode and no other mode (note that the most persistent mode is identical to the most amplifying mode in this case). In unconstrained networks (bottom), optimized initial conditions overlap strongly with the most amplifying mode and only weakly with other modes. (The non-zero overlap with the most persistent mode is simply due to the fact that there is a non-zero overlap between the most persistent and amplifying mode in random networks, and it is at the level that would be expected based on this overlap.) d, Time course of mean (across the 100 networks from c) absolute overlap with the persistent mode for 100-neuron symmetric (top) and unconstrained networks (bottom) when the network is started from optimized initial conditions (blue), and for comparison for the most amplifying (red dashed) initial conditions (cf. Fig. 2e). Note the close agreement between the two indicating that the most amplifying mode is indeed optimal in these networks. Horizontal black bar on x-axis shows the time period in which we applied the cost function to optimize the initial conditions (Methods). 
bioRxiv preprint doi: https://doi.org/10.1101/2021.11.16.468360; this version posted December 17, 2021. The copyright holder for this preprint (which was not certified by peer review) is the author/funder, who has granted bioRxiv a license to display the preprint in perpetuity. It is made available under aCC-BY 4.0 International license.

a
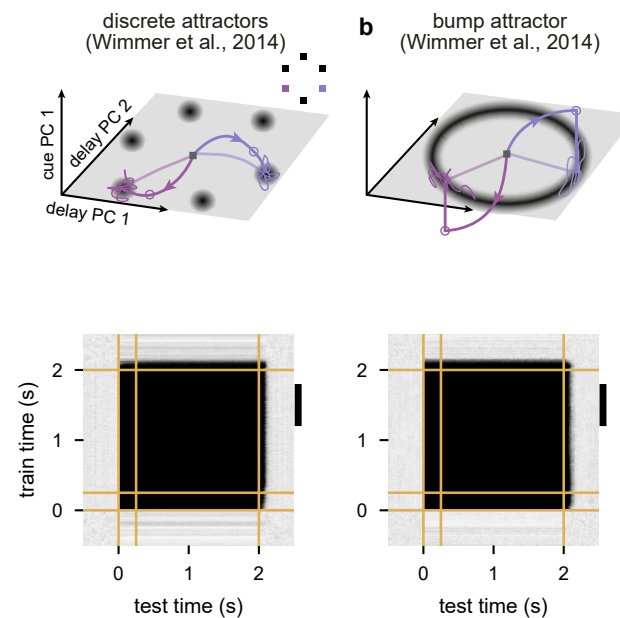

test time (s)

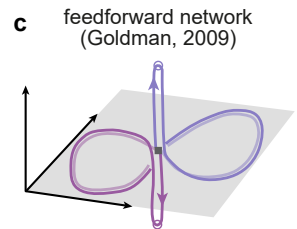

d integrator

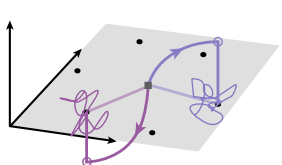

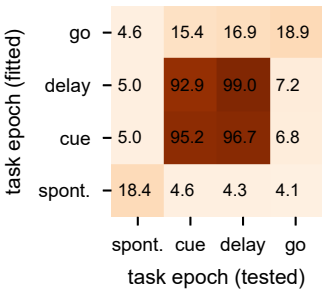
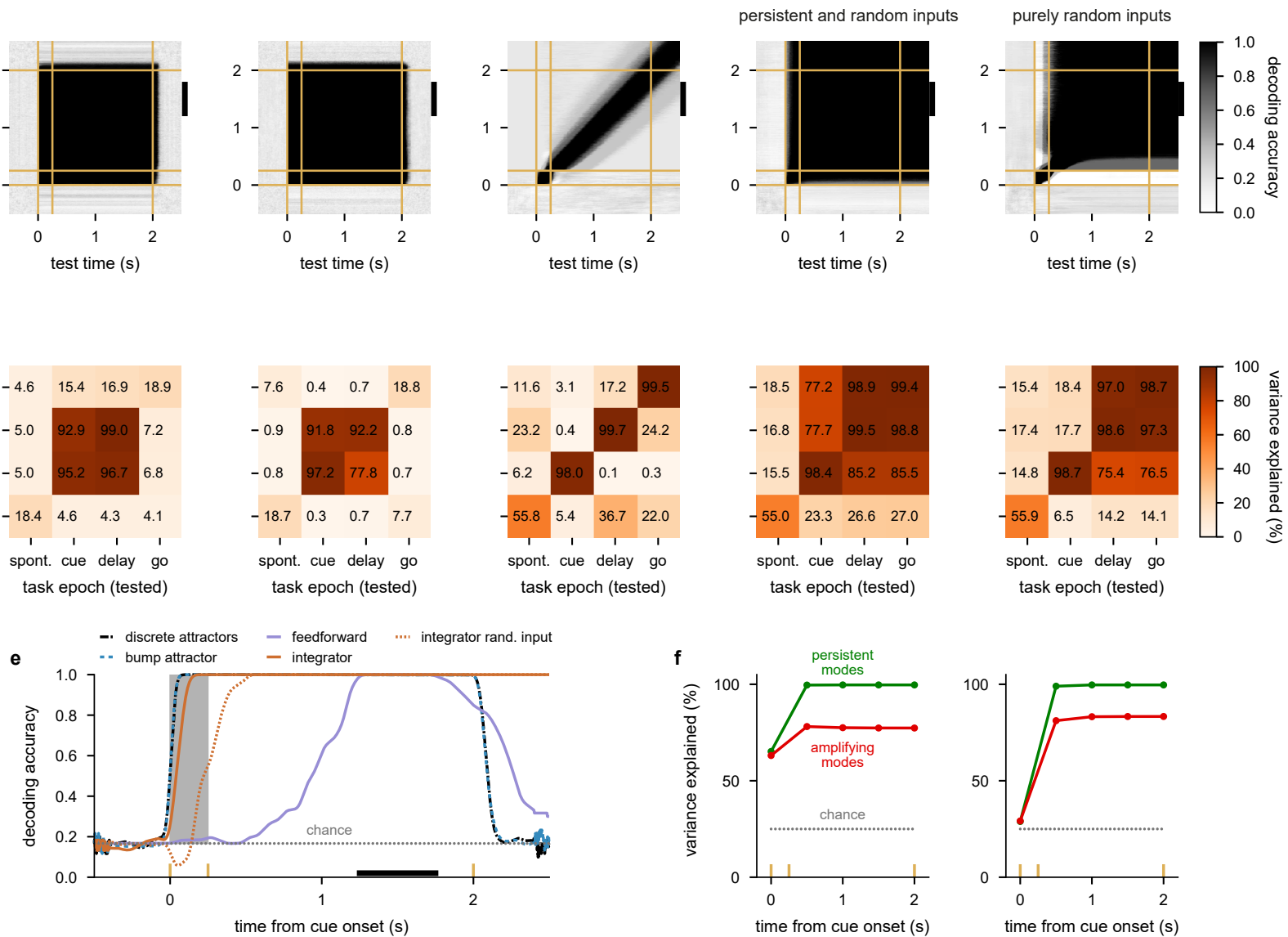

Extended Data Fig. 5 | Analysis of classical working memory models. a, Top: Schematic illustration of dynamics for a discrete multiattractor network model ${ }^{7,8,25}$ (see also Methods) in a 3-dimensional state space defined by the top 2 PCs of delay-epoch activity and the top PC of cue-epoch activity (orthogonalised with respect to the 2 delay PCs). We show state space trajectories for two cue conditions (bright purple curves). Grey squares indicate cue onset and open purple circles with arrows indicate cue offset. Pale curves show trajectories projected into the 2 delay PCs and thin lines indicate noisy delay-epoch activity. Shaded black regions indicate attractor states. Center: Cross-temporal decoding of model neural activity (cf. Fig. 3b and Fig. 4c). Yellow lines indicate cue onset, offset, and go times. The vertical black bar indicates the delay-trained decoder training time period from e. Bottom: Subspace overlap between different task epochs, measured as the percent variance explained (PVE) by projecting neural activity from one task epoch (tested) through the top 4 PCs of another task epoch (fitted; Methods, cf. Extended Data Fig. 6c, Extended Data Fig. 7d, Extended Data Fig. 8d, and Extended Data Fig. 10b). Diagonal elements show the PVE within each task epoch. $\mathbf{b}-\mathbf{d}$, Same as a but for a bump attractor model ${ }^{7}$ (b), a linear feedforward network ${ }^{18,33}$ (c), and a linear integrator model ${ }^{11}$ (d), respectively. In $\mathbf{d}$, we show results for inputs aligned either with both persistent and random directions (i.e. as in the original model formulation of Ref. 11; left column), or with purely random directions (right column). e, Performance of a delay-trained decoder (black bar indicates decoder training time period; Methods) on model neural activity over time for all 5 models (cf. Fig. 1f,k, Fig. 3a, and Fig. 4b). Yellow ticks on horizontal axis indicate cue onset, cue offset, and go cue times, and the vertical grey bar indicates the cue epoch. Grey dotted line shows chance level decoding. f, Percent variance explained by the subspace spanned by either the $25 \%$ most persistent (green) or $25 \%$ most amplifying (red) modes as a function of time for the linear integrator models from d (cf. Fig. $3 \mathrm{c}$ and Fig. $4 \mathrm{f}$ ). We show results for inputs aligned with both the persistent and random directions (left), or purely random directions (right). Grey dotted line shows chance level overlap with a subspace spanned by 25 random orthogonal directions. 
bioRxiv preprint doi: https://doi.org/10.1101/2021.11.16.468360; this version posted December 17, 2021. The copyright holder for this preprint (which was not certified by peer review) is the author/funder, who has granted bioRxiv a license to display the preprint in perpetuity. It is made available under aCC-BY 4.0 International license.
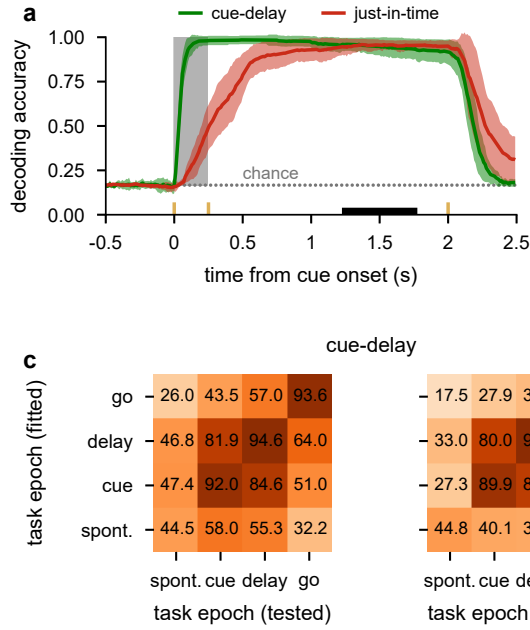

cue-delay
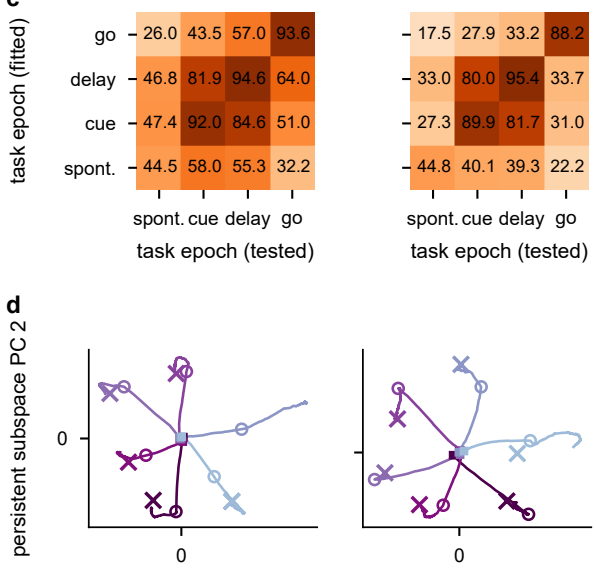

persistent subspace PC 1

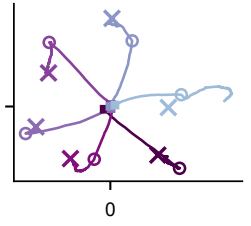

persistent subspace PC 1
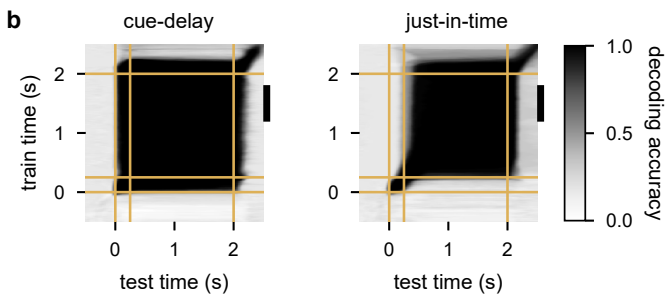

just-in-time
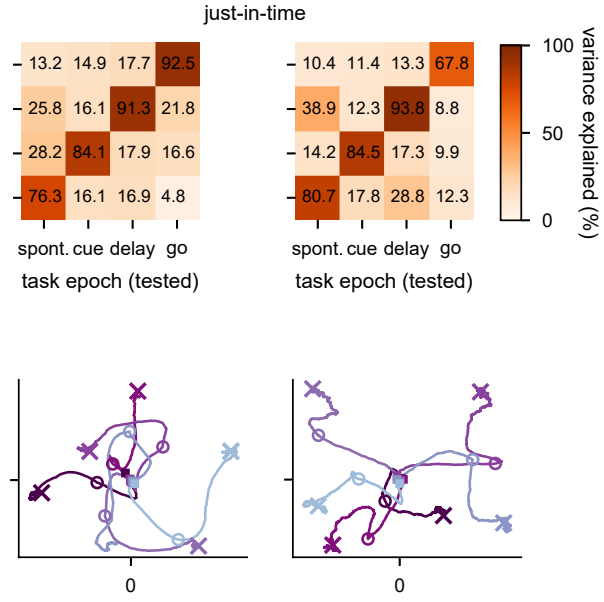

persistent subspace PC 1 persistent subspace PC 1

Extended Data Fig. 6 | Cue-delay and just-in-time trained networks. a-b, Same as Fig. 5c green and red, and Fig. 5d left and center, but with a regularisation strength of $\alpha_{\text {nonlin }}^{(2)}=0.0005$ used during training (Methods). c, Subspace overlap between different task epochs, measured as the percent variance explained (PVE) by projecting neural activity from one task epoch (tested) through the top 4 PCs of another task epoch (fitted; cf. Extended Data Fig. 5a-d, bottom, Extended Data Fig. 7d, Extended Data Fig. 8d, and Extended Data Fig. 10b). Diagonal elements show the PVE within each task epoch. We show results for cue-delay (left two panels) and just-in-time trained networks (right two panels) trained with either a regularisation strength of $\alpha_{\text {nonlin }}^{(2)}=0.00005$ (left panel for each model, as in Fig. 5) or $\alpha_{\text {nonlin }}^{(2)}=0.0005$ (right panel for each model, as in panels a-b). d, Neural activity plotted in the top two PCs of delay-epoch activity for all 6 initial conditions for cue-delay and just-in-time trained networks for each of the network-regularization combinations shown in c (Methods, cf. Extended Data Fig. 1b-d and f-h.) Purple traces show state-space trajectories, squares indicate cue onset, open circles indicate cue offset, and crosses indicate asymptotically stable fixed points, colours indicate cue condition as in Fig. 1e. 
bioRxiv preprint doi: https://doi.org/10.1101/2021.11.16.468360; this version posted December 17, 2021. The copyright holder for this preprint (which was not certified by peer review) is the author/funder, who has granted bioRxiv a license to display the preprint in perpetuity. It is made available under aCC-BY 4.0 International license.
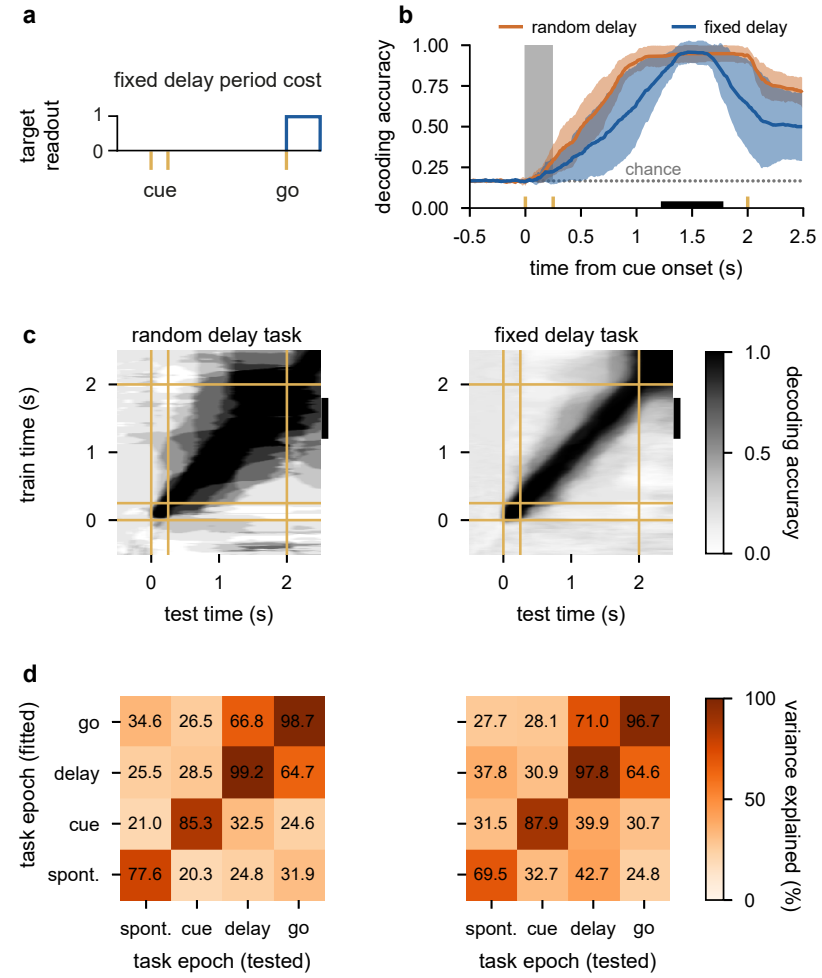

Extended Data Fig. 7 | After-go-time trained networks. a, Cost function for after-go-time training on the fixed delay task (Methods). Cue onset, cue offset, and go cue times are indicated by the yellow vertical lines. The boxcar shows the interval over which stable decoding performance was required (i.e. the cost was only applied after the go cue). b-c, Same as Fig. 5c orange and Fig. 5d right, but with a regularisation strength of $\alpha_{\text {nonlin }}^{(2)}=0.0005$ used during training and when either a random (b orange, $\mathbf{c}$ left) or a fixed delay task is used (b blue, $\mathbf{c}$ right, Methods). d, Subspace overlap between different task epochs, measured as the percent variance explained (PVE) by projecting neural activity from one task epoch (tested; cf. Extended Data Fig. 5a, bottom, Extended Data Fig. 6c, Extended Data Fig. 8d, and Extended Data Fig. 10b) through the top 4 PCs of another task epoch (fitted) for the networks shown in $\mathbf{b}-\mathbf{c}$. Diagonal elements show the PVE within each task epoch.
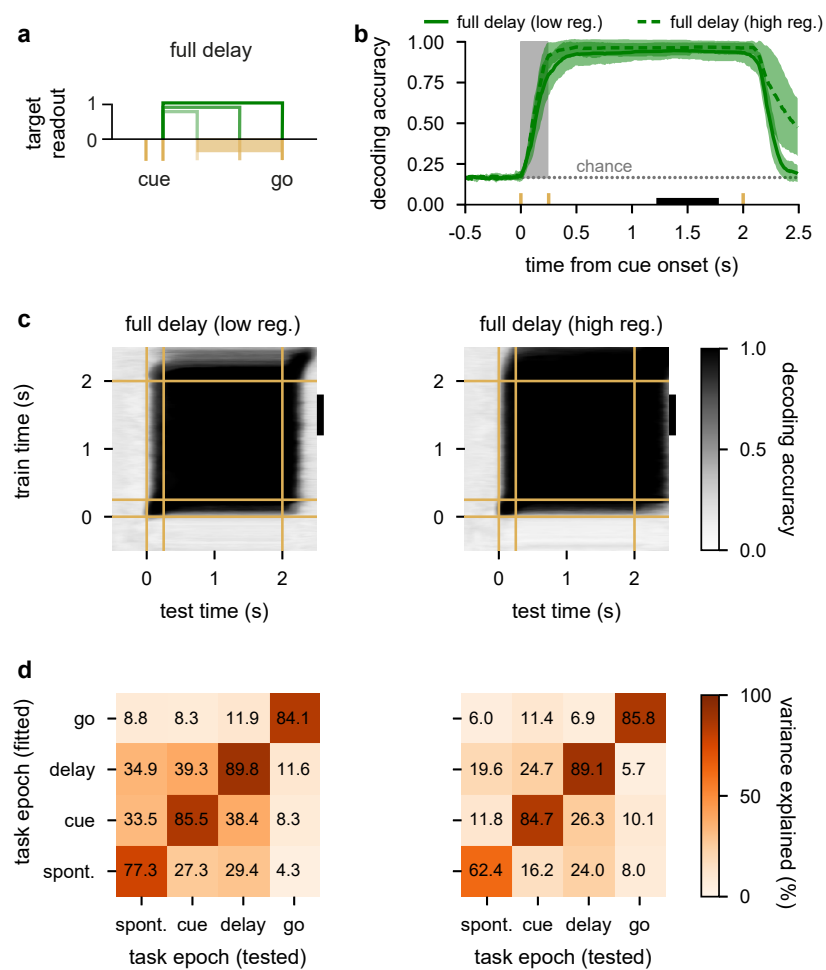

Extended Data Fig. 8 | Full-delay trained networks. a, Cost function for full-delay training on the random delay task (Methods). Yellow ticks indicate cue onset and offset times, the yellow bar indicates range of go times in the variable delay task. Boxcars show intervals over which stable decoding performance was required in three example trials with different delays (Methods). $\mathbf{b}-\mathbf{c}$, Same as Fig. $5 c-d$, but when training with the full-delay cost with a regularisation strength of $\alpha_{\text {nonlin }}^{(2)}=0.00005$ (b solid, c left) or $\alpha_{\text {nonlin }}^{(2)}=0.0005$ (b dashed, c right, Methods). d, Subspace overlap between different task epochs, measured as the percent variance explained (PVE) by projecting neural activity from one task epoch (tested; cf. Extended Data Fig. 5a, bottom, Extended Data Fig. 6c, Extended Data Fig. 7d, and Extended Data Fig. 10b) through the top 4 PCs of another task epoch (fitted) for the networks shown in b-c. Diagonal elements show the PVE within each task epoch. 
bioRxiv preprint doi: https://doi.org/10.1101/2021.11.16.468360; this version posted December 17, 2021. The copyright holder for this preprint (which was not certified by peer review) is the author/funder, who has granted bioRxiv a license to display the preprint in perpetuity. It is made available under aCC-BY 4.0 International license.

monkey $\mathrm{T}$
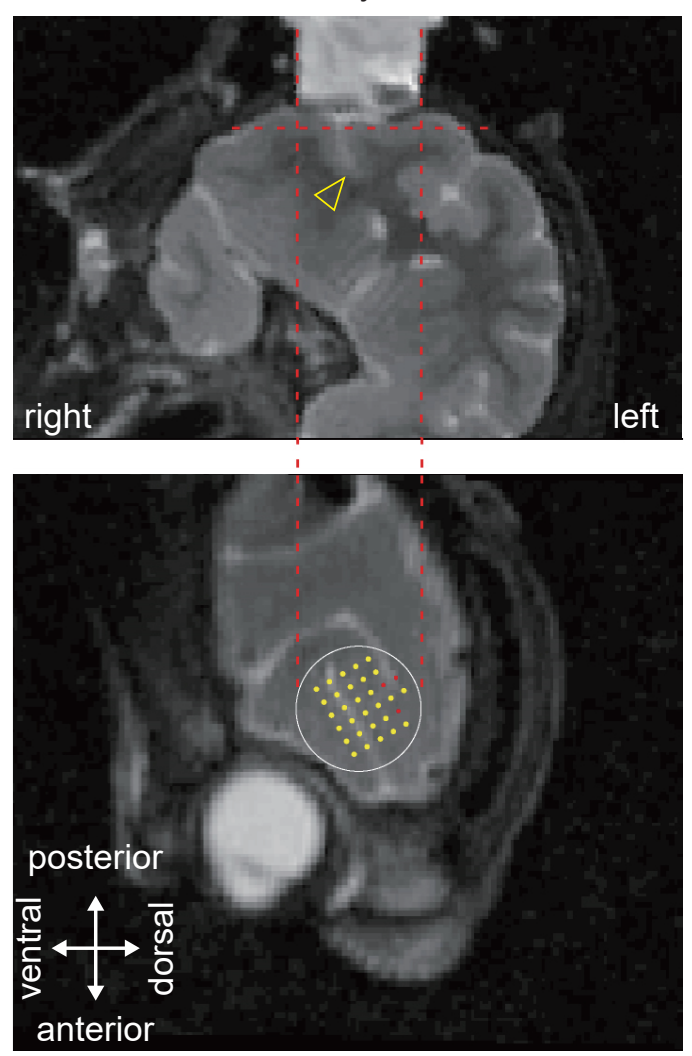

monkey K
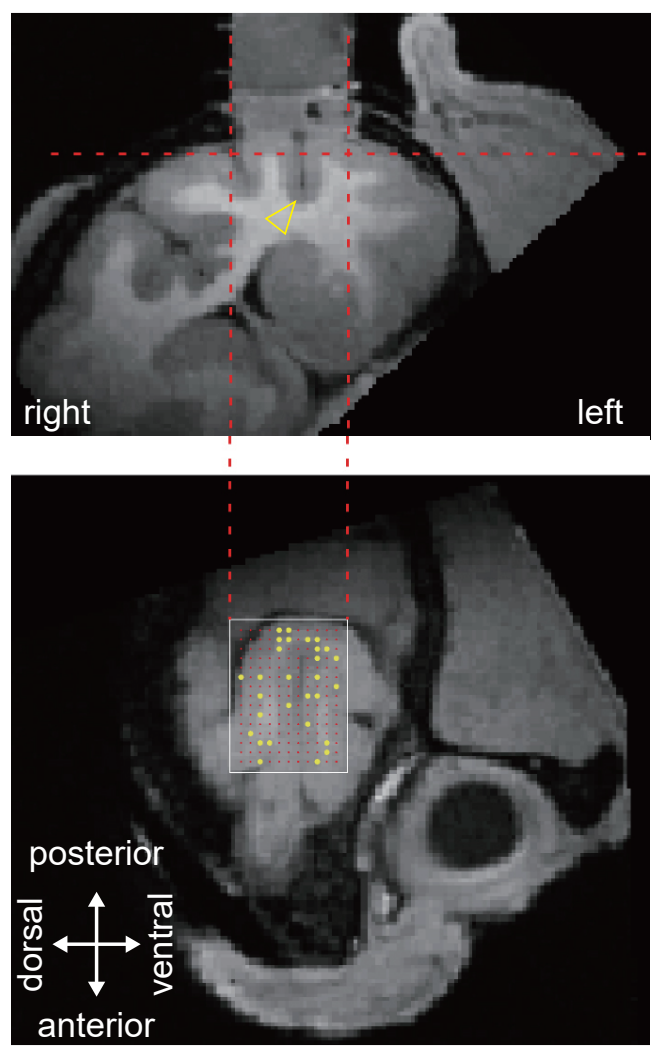

Extended Data Fig. 9 | Recording locations for the two monkeys. Left: recording locations in monkey K (T1-weighted image). In order to image the interior of the chamber, we filled the chamber with cut cottons soaked in iodine. In the upper picture, the yellow arrow indicates the principal sulcus. In the bottom picture, locations of the 11 by 15 grid holes were superimposed over the MR picture. Right: recording locations in monkey T (T2-weighted image). The bottom picture shows the location for the grid of the 32 semi-chronic electrodes. Yellow dots indicate electrode penetrations and recording sites, red dots indicate non-visited sites. 
bioRxiv preprint doi: https://doi.org/10.1101/2021.11.16.468360; this version posted December 17, 2021. The copyright holder for this preprint (which was not certified by peer review) is the author/funder, who has granted bioRxiv a license to display the preprint in perpetuity. It is made available under aCC-BY 4.0 International license.
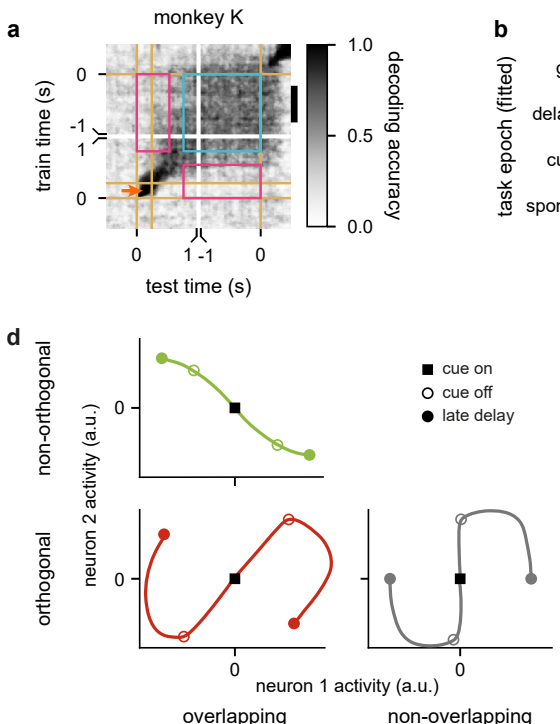
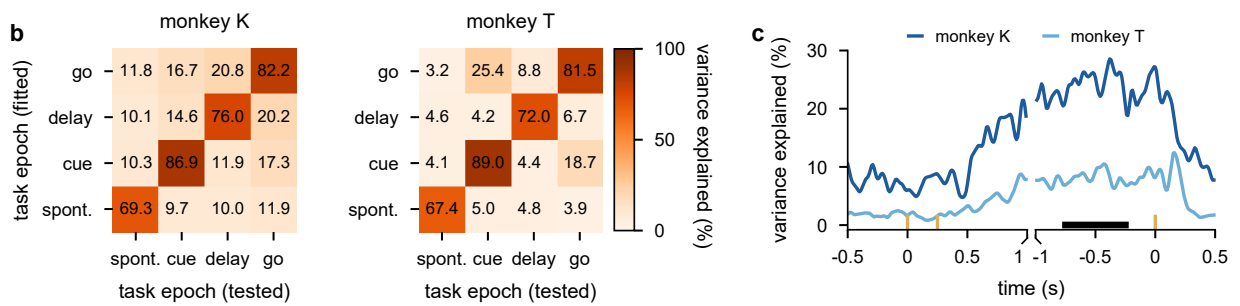

e

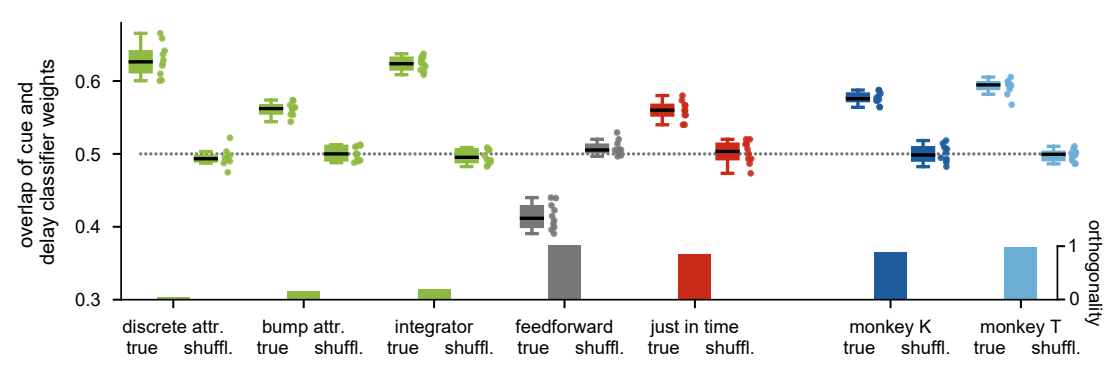

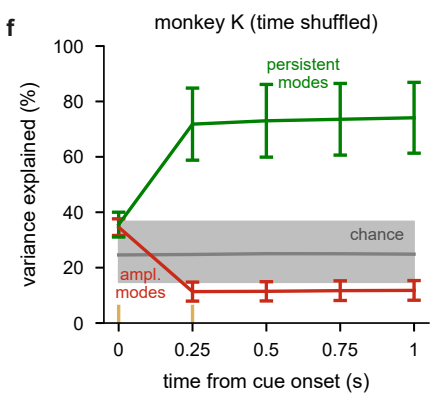
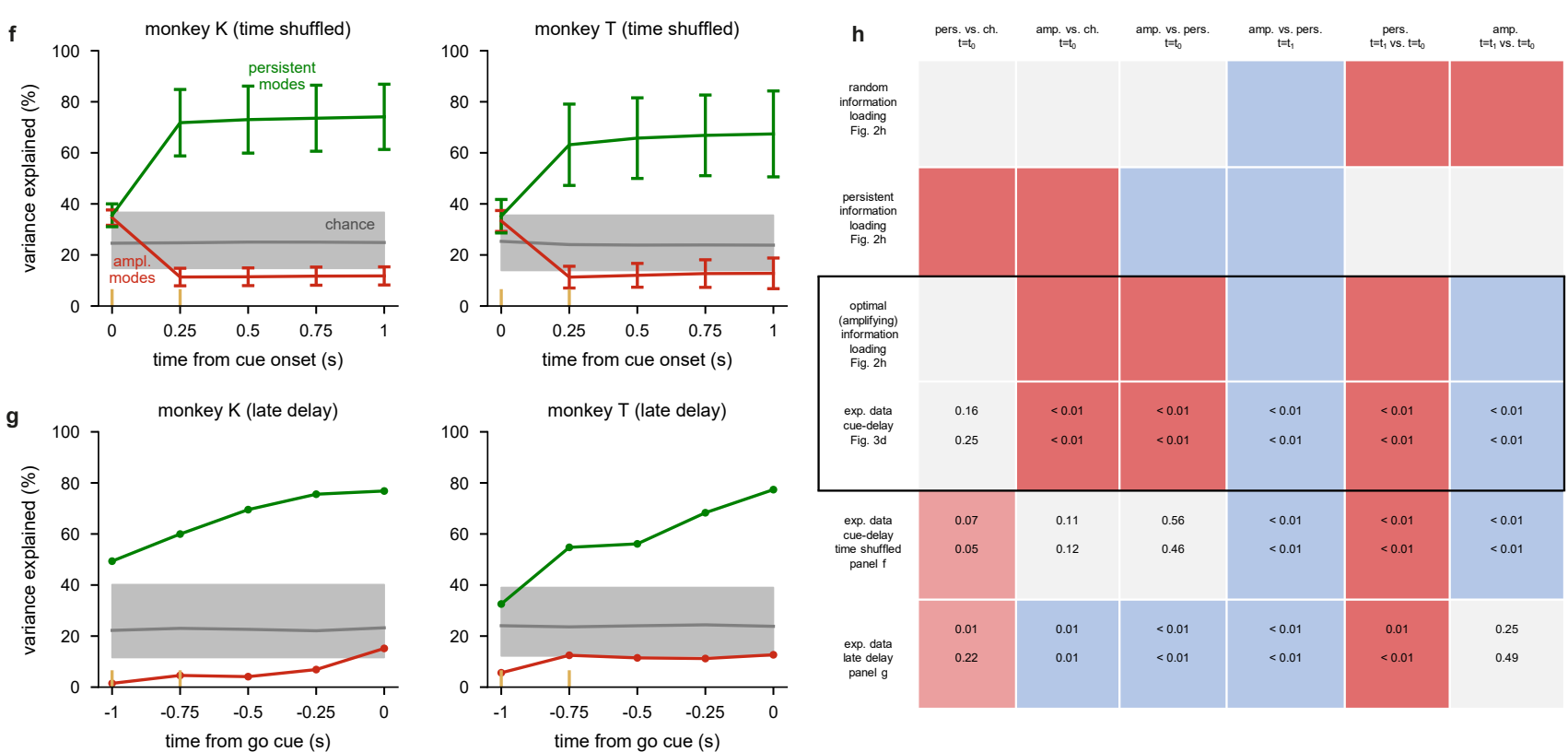

Extended Data Fig. 10 | Supplemental analysis of experimental data and comparison to models. a, Cross-temporal decoding analysis for monkey K (cf. Fig. 4c for the same analysis for monkey T and for explanation of plotting scheme and annotations). b, Subspace overlap between different task epochs, measured as the percent variance explained (PVE) by projecting neural activity from one task epoch (tested) through the top 10 PCs of another task epoch (fitted; cf. Extended Data Fig. 5a-d, bottom row). Diagonal elements show the PVE within each task epoch. We show results for monkey K (left) and monkey T (right). c, Time course of overlap with delay epoch subspace, measured as the percent variance explained by the top 2 PCs obtained from delay period activity (black bar shows time period of activity from which these PCs were obtained) on held-out test data taken in different time bins. This metric is called the alignment index ${ }^{64}$ and is very similar to that used in Ref. 11 (Methods). We show mean (over 10 different data splits) results for both monkeys. Yellow ticks on horizontal axis indicate cue onset, cue offset, and go times.

(Caption continued on next page.) 
bioRxiv preprint doi: https://doi.org/10.1101/2021.11.16.468360; this version posted December 17, 2021. The copyright holder for this preprint (which was not certified by peer review) is the author/funder, who has granted bioRxiv a license to display the preprint in perpetuity. It is made available under aCC-BY 4.0 International license.

Extended Data Fig. 10 | Supplemental analysis of experimental data and comparison to models (cont'd). d, Schematic of 3 different hypothetical scenarios for the relationship between cue and late delay activities (panels), illustrated in neural dynamics for 2 neurons and 2 cue conditions. Coloured curves show neural trajectories, black squares indicate cue onset, open circles indicate cue offset, and filled circles show late delay activity. Left vs. right: populations encoding the cue during cue and late delay periods are overlapping vs. non-overlapping, respectively. Top vs. bottom: cue and delay activities are non-orthogonal vs. orthogonal, respectively. (Note that we are not showing dynamics for non-overlapping, non-orthogonal dynamics because no overlap necessarily implies orthogonality.) e, Relationship between cue and late delay activities in various different models and our experimental recordings (x-axis). Top: population overlap measured as the mean difference between cue and delay epoch classifier weights (left for each model and data) and, as a control, when randomly shuffling classifier weights across neurons (right for each model and data) (Methods). Box plots show medians (black lines), quartiles (boxes), and 1.5 times the inter-quartile range (whiskers). Dotted grey line shows chance level overlap. Bottom: orthogonality measured as 1 minus the mean overlap between cue and delay epochs (given by the corresponding elements of the subspace overlap matrices shown in panel $\mathbf{b}$, Extended Data Fig. 5a-d, bottom row, and Extended Data Fig. 6c, center right). The discrete attractor, bump attractor, and integrator models show high overlap but low orthogonality. The simple feed-forward network shows high orthogonality but low overlap (note that recurrent networks with embedded feed-forward connectivity ${ }^{18}$ may show high overlap). The just-in-time network shows high overlap and orthogonality, similar to the experimental data in both monkeys. $\mathbf{f}-\mathbf{g}$, Same analysis as in Fig. $4 \mathrm{f}$, but either after randomly shuffling data across time (but consistently across conditions and neurons, and applied to the same time period as in the main analysis; $\mathbf{f}$, see also Methods), or applied to the late delay time period (without across-time shuffling) in which we do not expect information loading dynamics (g, see also Methods). h, Table showing $\mathrm{p}$-values (in each cell, top: monkey $\mathrm{K}$, bottom: monkey $\mathrm{T}$ ) from two-sided permutation tests for each comparison of the main analysis (row 4, repeated from the main text associated with Fig. 4d) and the control analyses shown in panels $\mathbf{f}$ and $\mathbf{g}$ of this figure (rows 5-6). Top 3 rows show predictions for the sign of each comparison under different information loading strategies in unconstrained linear networks (Fig. 3c, bottom): using inputs aligned with random directions (1st row), persistent directions (2nd row), or the most amplifying directions (3rd row). In the column headings, pers., amp., and ch. respectively refer to overlap with most persistent, most amplifying and random subspaces (chance), $t_{0}$ refers to the beginning of the analysis time window, i.e. cue onset (rows 1-5) or $1 \mathrm{~s}$ before the timing of the go cue (row 6 ), and $t_{1}=t_{0}+1 \mathrm{~s}$ refers to the end of the analysis time window. Grey indicates no significant difference between data points, red and blue indicate a significant difference for both monkeys where the first data point is respectively greater or smaller than the second data point, and pale red indicates a significant difference for one of the two monkeys (see Methods). 